\title{
Search for dark matter in association with a Higgs boson decaying to two photons at $\sqrt{s}=13 \mathrm{TeV}$ with the ATLAS detector
}

\author{
M. Aaboud et al. \\ (ATLAS Collaboration)
}

(Received 7 July 2017; published 8 December 2017)

\begin{abstract}
A search for dark matter in association with a Higgs boson decaying to two photons is presented. This study is based on data collected with the ATLAS detector, corresponding to an integrated luminosity of $36.1 \mathrm{fb}^{-1}$ of proton-proton collisions at the LHC at a center-of-mass energy of $13 \mathrm{TeV}$ in 2015 and 2016. No significant excess over the expected background is observed. Upper limits at $95 \%$ confidence level are set on the visible cross section for beyond the Standard Model physics processes, and the production cross section times branching fraction of the Standard Model Higgs boson decaying into two photons in association with missing transverse momentum in three different benchmark models. Limits at $95 \%$ confidence level are also set on the observed signal in two-dimensional mass planes. Additionally, the results are interpreted in terms of $90 \%$ confidence-level limits on the dark-matternucleon scattering cross section, as a function of the dark-matter particle mass, for a spin-independent scenario.
\end{abstract}

DOI: 10.1103/PhysRevD.96.112004

\section{INTRODUCTION}

The discovery of a particle consistent with the Standard Model (SM) Higgs boson in 2012 by the ATLAS [1] and CMS [2] collaborations has opened up new possibilities in searches for physics beyond the SM (BSM). Although strong astrophysical evidence $[3,4]$ indicates the possible existence of dark matter (DM), there is no evidence yet for nongravitational interactions between DM and SM particles. The interaction probability of DM particles, which are produced in SM particle collisions, with a detector is expected to be tiny. Thus, many searches for DM at the Large Hadron Collider (LHC) involve missing transverse momentum $\left(E_{\mathrm{T}}^{\text {miss }}\right)$ produced in association with detectable particles $\left(X+E_{\mathrm{T}}^{\text {miss }}\right.$ final states). In other $X+E_{\mathrm{T}}^{\text {miss }}$ searches in proton-proton ( $p p$ ) collisions, $X$ may represent a jet or a $\gamma / W / Z$ boson, which can be emitted directly from a light quark or gluon as initial-state radiation through SM gauge interactions. However, SM Higgs boson radiation from initial-state partons is highly suppressed, so events with a final state compatible with the production of a SM Higgs boson in association with $E_{\mathrm{T}}^{\text {miss }}$ can be sensitive probes of the structure of the BSM physics responsible for producing DM. The SM Higgs boson is expected to be produced from a new interaction between DM and the SM particles $[5,6]$. Both the ATLAS and CMS collaborations have previously searched for such topologies using

*Full author list given at the end of the article.

Published by the American Physical Society under the terms of the Creative Commons Attribution 4.0 International license. Further distribution of this work must maintain attribution to the author(s) and the published article's title, journal citation, and DOI.
$20.3 \mathrm{fb}^{-1}$ of $p p$ collision data at $\sqrt{s}=8 \mathrm{TeV}[7,8]$, and 2.3-36.1 $\mathrm{fb}^{-1}$ of $p p$ collision data at $\sqrt{s}=13 \mathrm{TeV}$ [9-11], considering the SM Higgs boson decay into a pair of photons or $b$-quarks in events with missing transverse momentum. Although the branching fraction of the SM Higgs boson decaying into a pair of photons is small, the diphoton system presented in this paper falls in a narrower mass range around the Higgs boson mass than in Ref. [11]. With the diphoton trigger, this channel is more sensitive in the low $E_{\mathrm{T}}^{\text {miss }}$ region than the channel with the SM Higgs boson decaying into a pair of $b$-quarks, which relies on the high $E_{\mathrm{T}}^{\text {miss }}$ trigger. This paper presents an updated search for DM particles $(\chi)$ associated with the SM Higgs boson $(h)$ decay to a pair of photons using $36.1 \mathrm{fb}^{-1}$ of $p p$ collision data collected at $\sqrt{s}=13 \mathrm{TeV}$ during 2015 and 2016, where both the integrated luminosity and the center-ofmass energy are significantly higher than in the previously published ATLAS analysis [7].

Three benchmark models are considered in this analysis. The leading-order (LO) Feynman diagrams representing the production of $h$ plus $E_{\mathrm{T}}^{\mathrm{miss}}$ in two simplified models [12] are shown in Figs. 1(a) and 1(b). In the first model, a massive vector mediator $Z^{\prime}$ emits a Higgs boson and subsequently decays to a pair of Dirac fermionic DM candidates. A vector-boson mediator arises in many BSM theories through a minimal extension to the gauge sector of the SM. In scenarios where the DM couples to the SM only via the $Z^{\prime}$ boson [i.e., the $Z_{B}^{\prime}$ model [5] represented in Fig. 1(a)], the associated $U^{\prime}(1)$ symmetry ensures the stability of the DM particle. The baryon number $B$ is associated with the gauge symmetry of $U(1)_{B}$, and an additional scalar particle (referred to as a baryonic Higgs boson) is introduced to break this symmetry spontaneously 


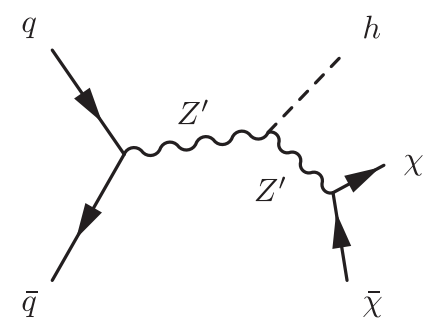

(a)

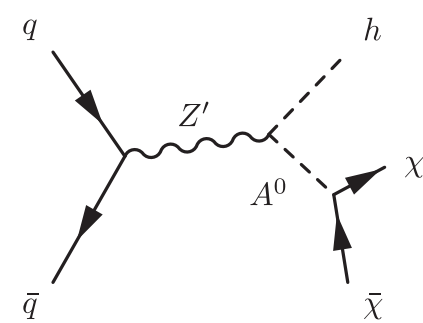

(b)

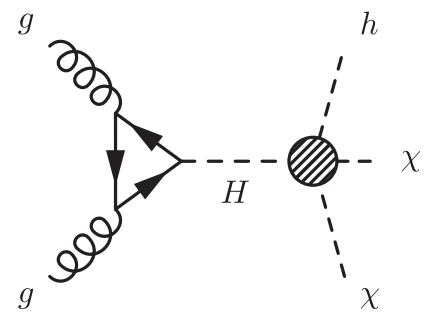

(c)

FIG. 1. The Feynman diagrams for the production of DM $(\chi)$ in association with a SM Higgs boson $(h)$ arising from three theoretical models considered in this paper: (a) $Z_{B}^{\prime}$ model, (b) $Z^{\prime}-2 \mathrm{HDM}$ model, (c) heavy-scalar model.

and generate the $Z^{\prime}$ boson mass (denoted by $m_{Z_{B}^{\prime}}$ ). The second model [from a $Z^{\prime}$-two-Higgs doublet model ( $Z^{\prime}$-2HDM) [13], Fig. 1(b)] involves the $Z^{\prime}$ boson decaying to the SM Higgs boson and an intermediate heavy pseudoscalar boson $A^{0}$, which then decays to a pair of Dirac fermionic DM particles. The minimum decay widths of the mediators are assumed for both the $Z_{B}^{\prime}$ and $Z^{\prime}-2 \mathrm{HDM}$ models to be the sum of the partial widths for all decays into DM and quarks that are kinematically accessible [12]. Alongside those simplified models recommended in Ref. [12], a third model [referred to as the heavy-scalar model [14], Fig. 1(c)] introduces a heavy scalar boson $H$ produced primarily via gluon-gluon fusion ( $\mathrm{ggF}$ ) with a mass in the range $2 m_{h}<m_{H}<2 m_{\text {top }}$, in which $m_{h}$ and $m_{\text {top }}$ represent the masses of the SM Higgs boson and top quark, respectively. The upper bound on $m_{H}$ is introduced to avoid a large branching fraction for $H \rightarrow t \bar{t}$, which would saturate the entire $H$ width leading to a $H \rightarrow h \chi \chi$ branching fraction close to zero. The lower bound on $m_{H}$ is required to be more than twice of $m_{h}$ to ensure the SM Higgs boson is produced on shell. An effective quartic coupling between $h, H$, and $\chi$ is considered, where the DM $\chi$ is assumed to be a scalar particle. The decay branching fraction of $H$ to $h$ and two $\chi$ particles is assumed to be $100 \%$ for this model, to simplify the interpretations. The DM mass $\left(m_{\chi}\right)$ is taken to be roughly half of the SM Higgs-boson mass to ensure on-shell decay of $H \rightarrow h \chi \chi$, and to suppress invisible decay modes of $h$, as described in Ref. [15]. While no assumptions are made here as to the nature of $H$, it can be viewed as a part of a 2HDM + $\chi$ scenario where $H$ may be considered as the $C P$-even heavy scalar boson [14].

This paper is organized as follows. Section II gives a brief description of the ATLAS detector. Section III describes the data set and the signal and background Monte Carlo (MC) simulation samples used. Section IV explains the event reconstruction, while Sec. V outlines the optimization of the event selection and categorization. Section VI summarizes the signal and background modeling. Section VII discusses the experimental and theoretical systematic uncertainties that affect the results. Section VIII presents the results and their interpretations, and finally a summary of the results is given in Sec. IX.

\section{ATLAS DETECTOR}

The ATLAS detector [16,17] is a multipurpose particle physics detector with approximately forwardbackward symmetric cylindrical geometry. ${ }^{1}$ The inner detector (ID) tracking system covers $|\eta|<2.5$ and consists of a silicon pixel detector, a silicon microstrip detector and a transition radiation tracker (TRT). The ID allows a precise reconstruction of charged-particle trajectories and of decay vertices of long-lived particles. The ID is surrounded by a thin superconducting solenoid providing a $2 \mathrm{~T}$ axial magnetic field. A high-granularity lead/liquid-argon (LAr) sampling calorimeter measures the energy and the position of electromagnetic showers in the central $(|\eta|<1.475)$ and endcap $(1.375<|\eta|<3.2)$ regions. It includes a presampler (for $|\eta|<1.8$ ) and three sampling layers up to $|\eta|<2.5$. The longitudinal and lateral segmentation of the calorimeter allows a measurement of the shower direction without assuming that the photon originates from a specific point along the beam line. LAr sampling calorimeters with copper and tungsten absorbers are also used to measure hadronic showers in the endcap $(1.5<|\eta|<3.2)$ and forward $(3.1<|\eta|<4.9)$ regions, while a steel/scintillator-tile calorimeter measures hadronic showers in the central region $(|\eta|<1.7)$. The muon spectrometer surrounds the calorimeters and consists of three large superconducting air-core toroid magnets, each with eight coils, a system of precision tracking chambers $(|\eta|<2.7)$, and fast tracking chambers for triggering $(|\eta|<2.4)$. Reconstructed events are selected by a two-level trigger system. The first-level trigger is hardware based, while the second-level trigger is implemented in software [18].

\footnotetext{
${ }^{1}$ ATLAS uses a right-handed coordinate system with its origin at the nominal interaction point (IP) in the center of the detector and the $z$ axis along the beam pipe. The $x$ axis points from the IP to the center of the LHC ring, and the $y$ axis points upward. Cylindrical coordinates $(r, \phi)$ are used in the transverse plane, $\phi$ being the azimuthal angle around the beam pipe. The pseudorapidity is defined in terms of the polar angle $\theta$ as $\eta=-\ln [\tan (\theta / 2)]$. The photon transverse energy is $E_{\mathrm{T}}=E / \cosh (\eta)$, where $E$ is its energy.
} 


\section{DATA AND SIMULATION SAMPLES}

The analysis uses $p p$ collision data with a bunch crossing interval of $25 \mathrm{~ns}$, collected in 2015 and 2016 at $\sqrt{s}=13 \mathrm{TeV}$. Only events that were recorded in stablebeam conditions, when relevant detector components were functioning properly, are considered. Events were collected using a diphoton trigger requiring two reconstructed photon candidates with transverse energies $\left(E_{\mathrm{T}}\right)$ of at least 35 and $25 \mathrm{GeV}$ for the $E_{\mathrm{T}}$-ordered leading and subleading photons, respectively. The trigger efficiency with respect to the offline-reconstructed photons, measured using the same method as described in Ref. [19], is $99 \%$. The data sample corresponds to an integrated luminosity of $36.1 \mathrm{fb}^{-1}$. There are on average 25 interactions in the same bunch crossing (in-time pileup) in this data sample.

The MC simulation of signal and background processes is used to optimize the selection criteria, estimate uncertainties, and study the shapes of the signal and background diphoton invariant mass $\left(m_{\gamma \gamma}\right)$ distributions. Signal events from $Z_{B}^{\prime}, Z^{\prime}-2 \mathrm{HDM}$, and the heavy-scalar models are generated using MADGRAPH_AMC@NLO 2.2.3 [20] at LO in quantum chromodynamics (QCD) using the NNPDF3.0LO [21] parton distribution function (PDF) set. Parton showering and hadronization are handled by the PYTHIA 8.186 [22] event generator with the A14 [23] set of tuned parameters (tune), using the NNPDF2.3LO PDF set [24]. MC samples for the $Z_{B}^{\prime}$ model are generated assuming a DM mass $m_{\chi}$ between 1 and $1000 \mathrm{GeV}$ and a range of mediator masses $m_{Z_{B}^{\prime}}$ between 1 and $2000 \mathrm{GeV}$. The upper bounds of the DM and mediator masses are motivated by the limited sensitivity with the current integrated luminosity, though in principle even heavier signals can be probed with this analysis. The values of the coupling constants and mixing parameter are chosen following the recommendations of the LHC dark matter forum [12]. The couplings of the $Z^{\prime}$ boson to DM particles $\left(g_{\chi}\right)$, to quarks $\left(g_{q}\right)$ and to the SM Higgs boson $\left(g_{h Z^{\prime} Z^{\prime}} / m_{Z^{\prime}}\right)$ are set to $1.0,1 / 3$, and 1.0 , respectively. The mixing angle between the baryonic Higgs boson and the SM Higgs boson is set to $\sin \theta=0.3$. The kinematic distributions predicted by the model are not strongly dependent on the coupling parameters after similar cuts either at the particle level or at the reconstruction level, and thus all samples are generated using this set of parameters. Similarly, the MC samples for the $Z^{\prime}$-2HDM model are generated for ranges of values of the mediator mass $m_{Z^{\prime}}=400$ to $1400 \mathrm{GeV}$ and pseudoscalar boson mass $m_{A^{0}}=200$ to $450 \mathrm{GeV}$ for which the search is sensitive. The masses of the neutral $C P$-even scalar $\left(H^{0}\right)$ and the charged scalars $\left(H^{ \pm}\right)$from $Z^{\prime}-2 H D M$ model are set to $300 \mathrm{GeV}$. The DM mass $m_{\chi}$ is set to $100 \mathrm{GeV}$, the ratio of the two-Higgs-doublet vacuum expectation values is set to $\tan \beta=1.0$ and the coupling constant between the $Z^{\prime}$, Higgs, and pseudoscalar bosons is set to $g_{Z^{\prime}}=0.8$, as suggested by Ref. [12]. The kinematics do not change appreciably when different values of $\tan \beta$ and $g_{Z^{\prime}}$ are used. Moreover, in the signal process, as the DM pairs are produced as a result of the $A^{0}$ decay, there are minimal kinematic changes when the $A^{0}$ is produced onshell. For the heavy-scalar model, the events are generated with $m_{H}$ in steps of $10 \mathrm{GeV}$ in the intervals $260 \leq m_{H} \leq$ $270 \mathrm{GeV}$ and $300 \leq m_{H} \leq 350 \mathrm{GeV}$ and in steps of $5 \mathrm{GeV}$ in the intervals $270 \leq m_{H} \leq 300 \mathrm{GeV}$ for $m_{\chi}=60 \mathrm{GeV}$. This mass value $\left(m_{\chi}=60 \mathrm{GeV}\right)$ was chosen in order to ensure on-shell decay of $H \rightarrow h \chi \chi$, and at the same time to suppress invisible decay modes of $h$. The impact of this choice on the expected sensitivity is negligible for $\chi$ mass from 50 to $70 \mathrm{GeV}$. The mass point $m_{H}=275 \mathrm{GeV}$ and $m_{\chi}=60 \mathrm{GeV}$ is taken as an example of the kinematics that depend on the value of $m_{H}$ in this model.

The dominant backgrounds are resonant $\mathrm{SM} h \rightarrow \gamma \gamma$, and nonresonant $\gamma \gamma, \gamma+$ jet, $W \gamma, Z \gamma, W \gamma \gamma$, and $Z \gamma \gamma$ production. For the resonant SM Higgs-boson production, events from $W h$ and $Z h$ processes are generated by PYTHIA 8.186 with the A14 tune and the NNPDF2.3LO PDF set. The ggF and vector-boson fusion (VBF) samples are generated by Powheg-Box 2 [25-28] interfaced to Pythia 8.186 with the AZNLO [29] tune and the CT10 PDF set [30]. Samples of $t \bar{t} h$ events are generated with MADGRAPH_AMC@NLO 2.2.3 [20] interfaced to PYTHIA 8.186 with the NNPDF3.0LO [21] PDF set. Samples of $b \bar{b} h$ events are generated by MADGRAPH_AMC@NLO 2.2.3 interfaced to PYTHIA 8.186 with the A14 tune and the NNPDF2.3LO PDF set. The nonresonant diphoton processes with associated jets are generated using SHERPA 2.1.1 [31] with the CT10 PDF set. Matrix elements are calculated with up to three partons at LO and merged with the SHERPA 2.1.1 parton shower [32] using the ME+PS@LO prescription [33]. The CT10 PDF set is used in conjunction with a dedicated parton-shower tuning developed by the SHERPA 2.1.1 authors. The $W \gamma, Z \gamma, W \gamma \gamma, Z \gamma \gamma$ samples are generated using SHERPA 2.1.1 with the CT10 PDF set.

The normalization of nonresonant backgrounds is obtained directly from data, as described in Sec. VI. The cross sections [34] of the SM Higgs-boson processes are calculated at next-to-leading order (NLO) in electroweak theory and next-to-next-to-leading order (NNLO) in QCD for VBF, $Z h$ and $W h$ samples and next-tonext-to-next-to-leading order plus next-to-next-to-leading logarithm $\left(\mathrm{N}^{3} \mathrm{LO}+\mathrm{NNLL}\right)$ in QCD for the $\mathrm{ggF}$ sample. The SM Higgs-boson mass is set to $125.09 \mathrm{GeV}$ [35] and its branching fraction decaying into two photons is $0.227 \%$ [34]. Samples for the $Z_{B}^{\prime}$ and $Z^{\prime}-2 H D M$ models are normalized using the LO cross sections predicted by MADGRAPH_AMC@NLO 2.2.3. Samples for the heavyscalar model are normalized using the cross section of a SM Higgs boson produced in ggF at the same mass at NNLO + NNLL in QCD.

Different pileup conditions from same and neighboring bunch crossings as a function of the instantaneous luminosity 
are simulated by overlaying minimum-bias events generated with PYTHIA 8.186 and EVTGEN [36] with the MSTW2008LO PDF set and the A2 [37] tune on the events of all hard processes. Differences between the simulated and observed distributions of the number of interactions per bunch crossing are removed by applying pileup weights to simulated events. Detector effects are simulated using a full simulation [38] performed using GEANT4 [39] for signals, SM Higgs processes, and $W \gamma, Z \gamma, W \gamma \gamma$, and $Z \gamma \gamma$ backgrounds. The diphoton continuum background and some of the signal samples are simulated using a fast simulation based on ATLFASTII [38].

\section{EVENT RECONSTRUCTION}

In each event, the observed final state is reconstructed from photons, leptons, jets, and $E_{\mathrm{T}}^{\text {miss }}$ that are built combining the related measurements provided by the various subdetectors of the experiment.

Photons are reconstructed from clusters of energy deposits in the electromagnetic calorimeter measured in projective towers. Clusters without matching tracks are classified as unconverted photon candidates. A photon is considered as a converted photon candidate if it is matched to a pair of tracks that pass a TRT-hits requirement [40] and that form a vertex in the ID which is consistent with originating from a massless particle, or if it is matched to a single track passing a TRT-hits requirement and having a first hit after the innermost layer of the pixel detector. The photon energy is calibrated using a multivariate regression algorithm trained with fully reconstructed MC samples and then corrections based on in situ techniques on data, as explained in Ref. [41]. The overall energy scale in data, as well as the difference in the constant term of the energy resolution between data and simulation, are estimated with a sample of $Z$ boson decays to electrons recorded in 2015 and 2016. The photon direction is estimated either using electromagnetic (EM) calorimeter longitudinal segmentation (if unconverted) or conversion vertex position (if converted), together with constraints from the $p p$ collision point

A "tight" photon identification requirement [40] is applied to reduce the misidentification of hadronic jets containing a high- $p_{\mathrm{T}}$ neutral hadron (e.g. $\pi^{0}$ ) decaying to two photons. The photon identification is based on the lateral profile of the energy deposits in the first and second layers of the electromagnetic calorimeter, and on the shower leakage fraction in the hadronic calorimeter. The selection requirements are tuned separately for unconverted and converted photon candidates. The identification efficiency of unconverted (converted) photons range from $85 \%$ to $95 \%$ (90\% to $98 \%$ ) between 25 and $200 \mathrm{GeV}$ [42]. Corrections are applied to the electromagnetic showershape variables for simulated photons, to account for small differences observed between data and simulation. The diphoton mass resolution at $m_{\gamma \gamma}=125 \mathrm{GeV}$ is in the range $1.32-1.86 \mathrm{GeV}$ [43].
To further reject hadronic backgrounds, requirements on two photon isolation variables are applied. The first variable, $E_{\mathrm{T}}^{\mathrm{iso}}$, is the sum of the transverse energies deposited in topological clusters [44] of cells in the calorimeter within a cone of size $\Delta R \equiv \sqrt{(\Delta \eta)^{2}+(\Delta \phi)^{2}}=0.2$ around each photon. The photon cluster energy and an estimate of the energy deposited by the photon outside its associated cluster are subtracted from that sum. To reduce the effects from the underlying event and pileup, the median ambient energy computed from low- $p_{\mathrm{T}}$ jets in the event $[45,46]$ is subtracted from $E_{\mathrm{T}}^{\text {iso }}$. Good candidates are required to have an $E_{\mathrm{T}}^{\text {iso }}$ less than $6.5 \%$ of the photon transverse energy. The second variable is a track-based isolation, defined as the scalar sum of the transverse momenta of all tracks with $p_{\mathrm{T}}>1 \mathrm{GeV}$ and consistent with originating from the primary vertex (PV) within a cone of size $\Delta R=0.2$ around each photon. In the case of converted photon candidates, the tracks associated with the conversion are removed. Good candidates are required to have a track isolation less than $5 \%$ of the photon transverse energy. The isolation efficiency for photons, which is mostly independent of their kinematic variables, is about $90 \%$ for the $\mathrm{ggF}$ SM Higgs boson process.

Selected events contain at least one PV formed from two or more tracks, each with $p_{\mathrm{T}}>400 \mathrm{MeV}$. In each event, the diphoton PV is chosen from the PV candidates using a neural network. The input variables to this neural network are the combined $z$-position of the intersections of the extrapolated photon trajectories with the beam axis; the sum of the squared transverse momenta $\sum p_{\mathrm{T}}^{2}$ and the scalar sum of the transverse momenta $\sum p_{\mathrm{T}}$ of the tracks associated with each reconstructed vertex; and the difference in azimuthal angle $\Delta \phi$ between the direction defined by the vector sum of the momenta of tracks from each vertex and that of the diphoton system. Dedicated studies of $Z \rightarrow e^{+} e^{-}$are performed in order to validate the diphoton vertex identification efficiency (correct identification of the hard process vertex by the neural network) between data and simulation. The method is similar to the one used in Ref. [47]. Studies show good agreement in diphoton vertex identification efficiency between data and simulation. The efficiency to locate the diphoton PV within $0.3 \mathrm{~mm}$ of the production vertex is $81 \%$ for SM Higgs boson production via ggF, $82 \%$ for a heavy-scalar signal with $m_{H}=$ $275 \mathrm{GeV}$ and scalar DM $m_{\chi}=60 \mathrm{GeV}, 67 \%$ for a $Z_{B}^{\prime}$ signal with $m_{Z_{B}^{\prime}}=200 \mathrm{GeV}$ and Dirac fermion DM $m_{\chi}=1 \mathrm{GeV}$, and $69 \%$ for a $Z^{\prime}-2 \mathrm{HDM}$ signal with $m_{Z^{\prime}}=1000 \mathrm{GeV}$, $m_{A^{0}}=200 \mathrm{GeV}$ and Dirac fermion DM $m_{\chi}=100 \mathrm{GeV}$.

Electrons are reconstructed from energy deposits measured in the EM calorimeter which are matched to ID tracks [48]. They are required to satisfy $|\eta|<2.47$, excluding the EM calorimeter transition region $1.37<|\eta|<1.52$, and to have $p_{\mathrm{T}}>10 \mathrm{GeV}$. The electrons are identified using a likelihood-based algorithm that uses the track and showershape variables as input. The "medium" criteria are applied, providing an identification efficiency varying from $85 \%$ to $95 \%$ as a function of $E_{\mathrm{T}}$ [49]. Loose calorimeter and track 
isolation requirements are applied to electrons. Pileup and the underlying event contributions in the calorimeter isolation are estimated using the ambient energy from low- $p_{\mathrm{T}}$ jets and are corrected on an event-by-event basis. In the inclusive diphoton sample the combined efficiency of the isolation requirements is $98 \%$ [50].

Muons are reconstructed from high-quality track segments in the muon spectrometer. In the region $|\eta|<2.5$, they must be matched to ID tracks. They are required to have $p_{\mathrm{T}}>10 \mathrm{GeV}$ and $|\eta|<2.7$ [51]. The muon "medium" criteria are applied and the identification efficiency is $96 \%$ [52]. The muon candidates must also satisfy calorimeter and track isolation criteria. The ambient energy from low- $p_{\mathrm{T}}$ jets is used to correct the contributions from pileup and the underlying event. The combined isolation efficiency varies from $95 \%$ to $99 \%$ as a function of $p_{\mathrm{T}}$ from $25 \mathrm{GeV}$ to $60 \mathrm{GeV}$ [52].

The significance of the track's transverse impact parameter with respect to the diphoton primary vertex, $\left|d_{0}\right| / \sigma_{d_{0}}$, is required to be less than 5.0 for electrons and 3.0 for muons. The longitudinal impact parameter $z_{0}$ must satisfy $\left|z_{0}\right| \sin \theta<0.5 \mathrm{~mm}$ for electrons and muons.

Jets are reconstructed from three-dimensional topological clusters using the anti- $k_{t}$ algorithm [53] with a radius parameter of $R=0.4$. The jets are required to have $p_{\mathrm{T}}>$ $20 \mathrm{GeV}$ and $|\eta|<4.5$ for the $E_{\mathrm{T}}^{\text {miss }}$ calculation and $p_{\mathrm{T}}>$ $25 \mathrm{GeV}$ and $|\eta|<4.4$ for the event selection. The jets with $|\eta|<2.4$ and $p_{\mathrm{T}}<60 \mathrm{GeV}$ must pass the jet vertex tagger selection [54], in which a jet is identified as originating from the diphoton primary vertex by examining the likelihood value calculated from the track information. In addition, quality criteria are applied to the jets, and events with jets consistent with noise in the calorimeter or noncollision backgrounds are vetoed [55].

The missing transverse momentum is calculated as the magnitude of the negative vectorial sum of the transverse momenta of calibrated photons, electrons, muons, and jets associated with the diphoton primary vertex. The transverse momenta of all tracks that originate from the diphoton primary vertex but are not already used in the $E_{\mathrm{T}}^{\text {miss }}$ calculation are summed and taken into account in the $E_{\mathrm{T}}^{\text {miss }}$ calculation. This term is defined as the track-based soft term [56,57]. Clusters and tracks not associated with the diphoton primary vertex are not included in the $E_{\mathrm{T}}^{\text {miss }}$ calculation, significantly suppressing the pileup effect and thus improving the $E_{\mathrm{T}}^{\text {miss }}$ resolution.

\section{EVENT SELECTION}

Events are required to have at least two photon candidates with $p_{\mathrm{T}}>25 \mathrm{GeV}$ and within a fiducial region of the EM calorimeter defined by $|\eta|<2.37$, excluding the region of $1.37<|\eta|<1.52$. Photon candidates in this fiducial region are ordered according to their $E_{\mathrm{T}}$ and only the first two are considered: the leading and subleading photon candidates must have $E_{\mathrm{T}}^{\gamma} / m_{\gamma \gamma}>0.35$ and 0.25 , respectively, where $m_{\gamma \gamma}$ is the invariant mass of the two selected photons. Events are required to have $105<m_{\gamma \gamma}<160 \mathrm{GeV}$ where the diphoton mass is calculated assuming that the photons originate from the diphoton primary vertex.

In the $Z_{B}^{\prime}$ and $Z^{\prime}$-2HDM models of DM production, the Higgs boson recoils against the DM pair, resulting in large $E_{\mathrm{T}}^{\text {miss }}$ in the event and large $p_{\mathrm{T}}$ of the diphoton candidate, denoted as $p_{\mathrm{T}}^{\gamma \gamma}$. By contrast, in the heavy-scalar model, the spectra of $E_{\mathrm{T}}^{\text {miss }}$ and $p_{\mathrm{T}}^{\gamma \gamma}$ are typically shifted to smaller values. Consequently, the classification of the selected events into categories depending on $E_{\mathrm{T}}^{\text {miss }}, p_{\mathrm{T}}^{\gamma \gamma}$ and other kinematic quantities leads to an increase in the overall sensitivity of the analysis to these different signal models.

The background events that survive the high- $E_{\mathrm{T}}^{\text {miss }}$ requirement but are not expected to present any genuine $E_{\mathrm{T}}^{\text {miss }}$ mostly have one or several high- $p_{\mathrm{T}}$ pileup jets. The misidentification of these jets therefore leads to large $E_{\mathrm{T}}^{\text {miss }}$. These pileup jets usually originate from a pileup vertex with larger $\Sigma p_{\mathrm{T}}^{2}$ than the diphoton primary vertex, where $p_{\mathrm{T}}$ is the track transverse momentum associated with a single vertex. Requiring the diphoton primary vertex to be the vertex with the largest $\Sigma p_{T}^{2}$ in each event helps to suppress pileup effects and reject a large fraction of the fake $E_{\mathrm{T}}^{\text {miss }}$ events. Models for which ggF is the main production mode typically pass this selection, since for example the heavy scalar produced in $\mathrm{ggF}$ is often accompanied by radiated jets, leading to a large $\Sigma p_{\mathrm{T}}^{2}$ of the vertex. An additional variable, $p_{\mathrm{T}}^{\text {hard }}$, defined as the magnitude of the vectorial sum of the transverse momenta photons and jets in the event, provides further discrimination against events with fake $E_{\mathrm{T}}^{\text {miss }}$ in the low- $E_{\mathrm{T}}^{\text {miss }}$ region. To further reject the background events from SM $V \gamma$ and $V \gamma \gamma$ production (where $V$ stands for $W$ or $Z$ ), which contribute significantly in the high- $E_{\mathrm{T}}^{\text {miss }}$ region, a lepton (electron or muon) veto is applied.

The selected events are thus divided into five categories based on the following:

(i) the $E_{\mathrm{T}}^{\text {miss }}$ significance, $S_{E_{\mathrm{T}}^{\text {miss }}}=E_{\mathrm{T}}^{\text {miss }} / \sqrt{\sum E_{\mathrm{T}}}$, where the total transverse energy $\sum E_{\mathrm{T}}$ is calculated from the scalar sum of the transverse momenta of

TABLE I. Optimized criteria used in the categorization. The categories are defined sequentially in the rows and each category excludes events in the previous row.

\begin{tabular}{ll}
\hline \hline Category & \multicolumn{1}{c}{ Requirements } \\
\hline Mono-Higgs & $S_{E_{\mathrm{T}}^{\text {miss }}}>7 \sqrt{\mathrm{GeV}}, p_{\mathrm{T}}^{\gamma \gamma}>90 \mathrm{GeV}$, lepton veto \\
High- $E_{\mathrm{T}}^{\text {miss }}$ & $S_{E_{\mathrm{T}}^{\text {miss }}}>5.5 \sqrt{\mathrm{GeV}}, \mathrm{PV}^{\text {highest }}=\mathrm{PV}^{\gamma \gamma}$ \\
Intermediate- $E_{\mathrm{T}}^{\text {miss }}$ & $S_{E_{\mathrm{T}}^{\text {miss }}}>4 \sqrt{\mathrm{GeV}}, p_{\mathrm{T}}^{\text {hard }}>40 \mathrm{GeV}$, \\
& $\mathrm{PV}^{\text {highest }}=\mathrm{PV}{ }^{\gamma \gamma}$ \\
Different-Vertex & $S_{E_{\mathrm{T}}^{\text {miss }}}>4 \sqrt{\mathrm{GeV}}, p_{\mathrm{T}}^{\text {hard }}>40 \mathrm{GeV}$, \\
& $\mathrm{PV}^{\text {highest }} \neq \mathrm{PV} \gamma \gamma$ \\
Rest & $p_{\mathrm{T}}^{\gamma \gamma}>15 \mathrm{GeV}$ \\
\hline \hline
\end{tabular}


the calibrated photons, electrons, muons and jets used in the $E_{\mathrm{T}}^{\text {miss }}$ calculation described in Sec. IV, as well as the tracks not associated with these but the PV;

(ii) the diphoton transverse momentum, $p_{\mathrm{T}}^{\gamma \gamma}$;

(iii) $p_{\mathrm{T}}^{\text {hard }}$;

(iv) the number of leptons in the event;

(v) the diphoton vertex is the highest $\Sigma p_{\mathrm{T}}^{2}$ vertex.

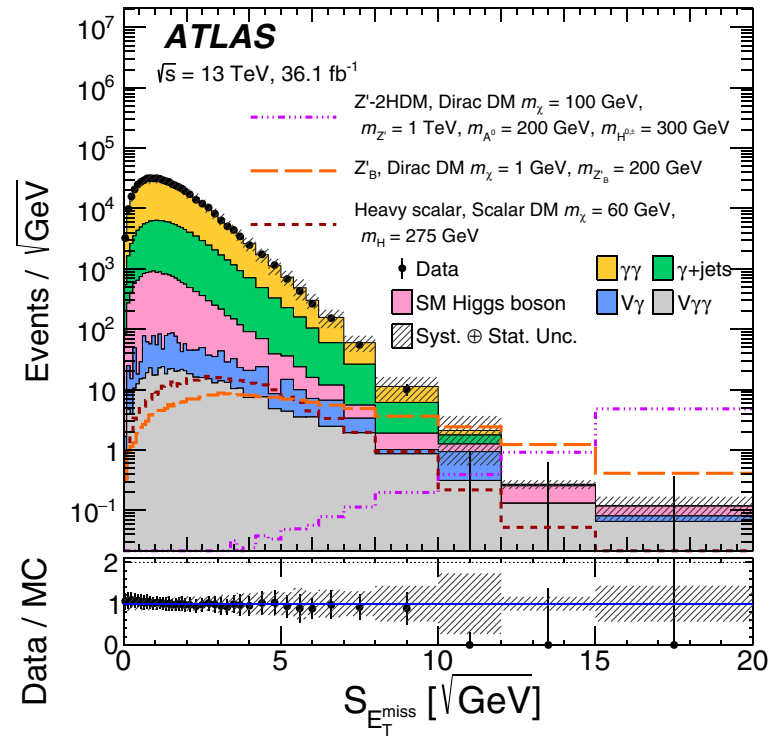

(a)
The resulting categorization scheme is shown in Table I. The categories are defined sequentially in the rows and each category excludes events in the previous row. The $Z_{B}^{\prime}$ and $Z^{\prime}-2$ HDM signal samples are used to optimize the definition of the Mono-Higgs category, which provides most of the sensitivity to those two models. The other four categories, which provide extra

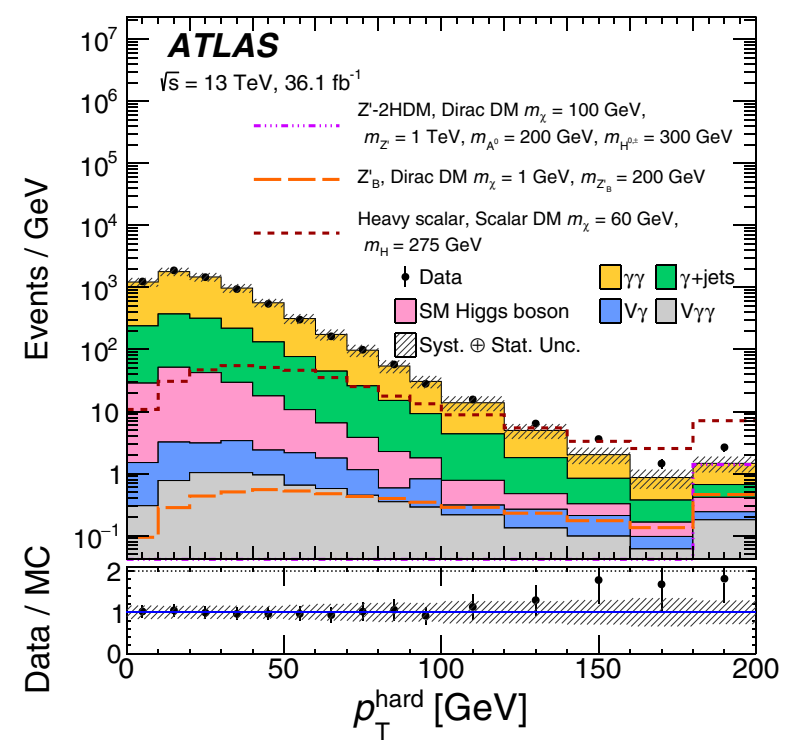

(c)

FIG. 2. The distribution of (a) $S_{E_{\mathrm{T}}^{\text {miss }}}$, (b) $p_{\mathrm{T}}^{\gamma \gamma}$, and (c) $p_{\mathrm{T}}^{\text {hard }}$ after the selection of diphoton candidates in $120<m_{\gamma \gamma}<130 \mathrm{GeV}$. Expected distributions are shown for a $Z_{B}^{\prime}$ signal with $m_{Z_{B}^{\prime}}=200 \mathrm{GeV}$ and Dirac fermion DM $m_{\chi}=1 \mathrm{GeV}$; a $Z^{\prime}-2 \mathrm{HDM}$ signal with $m_{Z^{\prime}}=1000 \mathrm{GeV}, m_{A^{0}}=200 \mathrm{GeV}$ and Dirac fermion DM $m_{\chi}=100 \mathrm{GeV}$; and a heavy-scalar model with $m_{H}=275 \mathrm{GeV}$ and scalar $\mathrm{DM} m_{\chi}=60 \mathrm{GeV}$. These overlaid signal points are representative of the model kinematics. Only the quadratic sum of the MC statistical and experimental systematic uncertainties in the total background is shown as the hatched bands, while the theoretical uncertainties in the background normalization are not included. Overflow events are included in the rightmost bin. The asymmetric error bars on data points come from Poissonian confidence intervals at $68 \%$ confidence level. 
sensitivity to heavy scalar boson events with softer $E_{\mathrm{T}}^{\text {miss }}$, are optimized using simulated heavy scalar boson samples to cover the different kinematic regimes of the heavy-scalar model.

Figure 2 shows the distributions of $S_{E_{\mathrm{T}}^{\text {miss }}}, p_{\mathrm{T}}^{\text {hard }}$, and $p_{\mathrm{T}}^{\gamma \gamma}$ after the selection of diphoton candidates in $120<m_{\gamma \gamma}<130 \mathrm{GeV}$. These distributions illustrate three kinds of signals in different $S_{E_{\mathrm{T}}^{\text {miss }}}$ regimes, and the contributions from the different background processes. Expected distributions are shown for a $Z_{B}^{\prime}$ signal with $m_{Z_{B}^{\prime}}=200 \mathrm{GeV}$ and Dirac fermion DM $m_{\chi}=1 \mathrm{GeV}$, a $Z^{\prime}$-2HDM signal with $m_{Z^{\prime}}=1000 \mathrm{GeV}, m_{A^{0}}=200 \mathrm{GeV}$ and Dirac fermion DM $m_{\chi}=100 \mathrm{GeV}$, and a heavy-scalar model with $m_{H}=275 \mathrm{GeV}$ and scalar DM $m_{\chi}=60 \mathrm{GeV}$. These overlaid signal points are representative of the model kinematics.

For the distributions shown in Fig. 2, the simulation is used to determine the shapes and normalizations of the $V \gamma$ and $V \gamma \gamma$ contributions, as well as the shape of the $\gamma \gamma$ contribution, respectively. The normalizations of the $\gamma \gamma$ and $\gamma+$ jet contributions are fixed to $79 \%$ and $19 \%$ of the data yield, where these fractions are estimated from a twodimensional sideband technique by counting the number of events in which one or both photons pass or fail the identification or isolation requirements [58]. The shape of the $\gamma+$ jet contribution is determined from a data control region where one photon fails to satisfy the photon identification criteria, after subtracting the contamination expected from $\gamma \gamma, V \gamma$ and $V \gamma \gamma$.

The slight discrepancies observed in the distributions of $S_{E_{\mathrm{T}}^{\text {miss }}}, p_{\mathrm{T}}^{\gamma \gamma}$, and $p_{\mathrm{T}}^{\text {hard }}$ in Fig. 2 do not affect the results. Discrepancies are found mainly in nonresonant backgrounds, which are estimated directly from data, as explained in Sec. VI.

\section{SIGNAL AND BACKGROUND PARAMETRIZATION}

The signal and backgrounds are extracted by fitting analytic functions to the diphoton invariant mass distribution in each category. For the signal and the SM Higgsboson background, the expected normalizations are obtained from their theoretical cross sections multiplied by the product of the acceptance times efficiency from the simulation. The shapes are modeled with a double-sided crystal ball function (as defined in Ref. [43]). The shape parameters are determined by fitting the diphoton mass distribution in simulation for each category.

Both the normalization and the shape of the nonresonant background are obtained by fitting the diphoton invariant mass distribution in data for each category. A variety of analytic functions are considered for the nonresonant background parametrization: exponential functions of different-order polynomials, Bernstein polynomials of different order, and an adapted dijet function [59]. The potential bias associated with the choice of a specific analytic function to model the continuum background (referred to as the nonresonant background modeling uncertainty, $\Delta N_{\mathrm{bkg}}^{\text {nonres }}$ ) is estimated for each category as the signal event yield extracted from a signal-plus-background maximum-likelihood fit to a background-only diphoton invariant mass distribution with small statistical fluctuations [43]. The backgroundonly distribution is obtained by mixing simulated $\gamma \gamma$, $\gamma+$ jet, $V \gamma$, and $V \gamma \gamma$ processes. The samples of $V \gamma$ and $V \gamma \gamma$ are weighted according to their theoretical cross section while $\gamma \gamma$ and $\gamma+$ jet samples are normalized to the number of candidates in data in the mass window $105<m_{\gamma \gamma}<160 \mathrm{GeV}$ scaled by the fraction of each sample (79\% for $\gamma \gamma$ and $19 \%$ for $\gamma+$ jet). For a given functional form, several fits are tested by varying the position of the signal peak between 115 and $135 \mathrm{GeV}$. The largest number of signal events obtained in these fits to the background-only templates is taken as $\Delta N_{\mathrm{bkg}}^{\text {nonres }}$. Among the different analytic functions that were tested, the parametrization with the smallest $\Delta N_{\mathrm{bkg}}^{\text {nonres }}$, or the minimum number of free parameters when the same $\Delta N_{\mathrm{bkg}}^{\text {nonres }}$ values are obtained, is selected as the nominal background parametrization to describe the nonresonant background shape. In addition, a $\chi^{2}$ test is performed to ensure that the fit is compatible with the data in each category.

The sidebands $\left(105<m_{\gamma \gamma}<120 \mathrm{GeV}\right.$ and $130<m_{\gamma \gamma}<$ $160 \mathrm{GeV}$ ) of data and the simulated events are compared with each other. Due to the low statistics in the Mono-Higgs category, the potential discrepancies between data and the simulated events are obscured by the statistical uncertainties. A visible discrepancy is only observed in the high- $E_{\mathrm{T}}^{\text {miss }}$, intermediate- $E_{\mathrm{T}}^{\text {miss }}$, different-vertex, and rest categories with large data statistics and in these cases a data-driven reweighting is applied to the simulated events to correct the shape. In order to check whether there is a substantial improvement in $\chi^{2}$ between two nested functions, several $F$ tests [19] are performed in the data sidebands of the Mono-Higgs category where the simple exponential function is chosen. The $F$ tests are performed by comparing the simple exponential function to other higher-order functions. A test statistic $F$ is calculated by using the resulting values of $\chi^{2}$, and its probability is compared with that expected from a Fisher distribution with the corresponding number of degrees of freedom. The hypothesis that a higher-order function is not needed is rejected if the $F$-test probability is less than 5\%. The tests show that the higher-order functions do not provide a significantly better fit and the lower-order function is sufficient. The selected analytic function along with its nonresonant background modeling uncertainty in each category, which is taken as an estimate of the systematic uncertainty due to the choice of parametrization, is shown in Table II. 
TABLE II. The analytic functions used to model the nonresonant $m_{\gamma \gamma}$ distribution, the uncertainty in the signal due to the nonresonant background modeling $\left(\Delta N_{\mathrm{bkg}}^{\text {nonres }}\right)$ and the relative uncertainties in the expected nonresonant background and signal per category. The relative uncertainty in the signal is evaluated in the Mono-Higgs category by using the $Z_{B}^{\prime}$ yields and in the other categories by using the heavy-scalar yields as $N_{\text {signal }}$ from Table IV. The signal yields are calculated in the $m_{\gamma \gamma}$ range between 120 and $130 \mathrm{GeV}$. The variable $x$ is defined as $m_{\gamma \gamma} / \sqrt{s}$ while $a$ and $b$ are parameters of the background functions. For the rest category, $C_{3}^{j}$ are binomial coefficients and $b_{j, 3}$ are Bernstein coefficients.

\begin{tabular}{llccc}
\hline \hline Category & \multicolumn{1}{c}{ Function } & $\Delta N_{\mathrm{bkg}}^{\text {nonres }}$ & $\Delta N_{\mathrm{bkg}}^{\text {nonres }} / N_{\mathrm{bkg}}^{\text {nonres }}[\%]$ & $\Delta N_{\mathrm{bkg}}^{\text {nonres }} / N_{\text {signal }}[\%]$ \\
\hline Mono-Higgs & $\exp (a \cdot x)$ & 1.2 & 9.8 & 6.0 \\
High- $E_{\mathrm{T}}^{\text {miss }}$ & $\left(1-x^{1 / 3}\right)^{b} \cdot x^{a}$ & 2.7 & 4.0 & 11 \\
Intermediate- $E_{\mathrm{T}}^{\text {miss }}$ & $\exp \left(a \cdot x+b \cdot x^{2}\right)$ & 5.8 & 1.3 & 14 \\
Different-vertex & $\exp \left(a \cdot x+b \cdot x^{2}\right)$ & 8.4 & 0.5 & 26 \\
Rest & $\sum_{j=0}^{3} C_{3}^{j} x^{j}(1-x)^{3-j} b_{j, 3}$ & 61 & $<0.1$ & 28 \\
\hline \hline
\end{tabular}

\section{SYSTEMATIC UNCERTAINTIES}

Uncertainties from experimental and theoretical sources affect the signal efficiency and the SM Higgs-boson background yield estimated from the simulated MC samples. The nonresonant background is obtained directly from the fit to the data. The only systematic uncertainty in the nonresonant background is the potential bias $\Delta N_{\mathrm{bkg}}^{\text {nonres }}$. A summary of the experimental and theoretical uncertainties with respect to the yield of the background from SM Higgs-boson processes, nonresonant background, and signal production is shown in Table III.

The uncertainty in the combined 2015 and 2016 integrated luminosity is $3.2 \%$. It is derived, following a methodology similar to that detailed in Ref. [60], from a calibration of the luminosity scale using $x-y$ beam-separation scans performed in August 2015 and May 2016.

The efficiency of the diphoton trigger used to select events is evaluated in MC simulation using a trigger matching technique and in data using a bootstrap method [18]. In the diphoton invariant mass window of $105<m_{\gamma \gamma}<160 \mathrm{GeV}$, the trigger efficiency uncertainty is found to be $0.4 \%$.

The uncertainty in the vertex selection efficiency is assessed by comparing the efficiency of finding photonpointing vertices in $Z \rightarrow e^{+} e^{-}$events in data and $\mathrm{MC}$ simulation [19], for which each electron track is removed

TABLE III. Breakdown of the dominant systematic uncertainties in the range of $105<m_{\gamma \gamma}<160 \mathrm{GeV}$. The uncertainties in the yield of signals, the background from SM Higgs-boson processes, and nonresonant background are shown. All production modes of the SM Higgs boson are considered together. Values for the impact on all categories are shown, unless one of the systematic uncertainties is not applicable to the sample, in which case the value is substituted by a "...". If a given source has a different impact on the various categories, the given range corresponds to the smallest and largest impacts among categories or among the different signal models used in the analysis. In addition, the potential bias coming from nonresonant background mismodeling is shown relative to the background.

\begin{tabular}{|c|c|c|c|}
\hline \multirow[b]{2}{*}{ Source } & \multirow[b]{2}{*}{ Signals [\%] } & \multicolumn{2}{|c|}{ Backgrounds [\%] } \\
\hline & & SM Higgs boson & Non-resonant background \\
\hline \multicolumn{4}{|l|}{ Experimental } \\
\hline Luminosity & \multicolumn{2}{|c|}{3.2} & $\cdots$ \\
\hline Trigger efficiency & \multicolumn{2}{|c|}{0.4} & $\cdots$ \\
\hline Vertex selection & \multicolumn{2}{|c|}{$<0.1$} & $\cdots$ \\
\hline Photon energy scale & $0.1-2.0$ & $0.1-1.4$ & $\cdots$ \\
\hline Photon energy resolution & $0.1-0.2$ & $0.1-1.1$ & $\cdots$ \\
\hline Photon identification efficiency & $2.9-4.3$ & $1.9-3.8$ & $\cdots$ \\
\hline Photon isolation efficiency & 1.2 & $0.8-1.6$ & $\cdots$ \\
\hline$E_{\mathrm{T}}^{\mathrm{miss}}$ reconstruction (diphoton vertex) & $<0.1$ & $0.5-1.9$ & $\cdots$ \\
\hline$E_{\mathrm{T}}^{\mathrm{miss}}$ reconstruction (jets, soft term) & $1.0-1.4$ & $0.8-23$ & $\cdots$ \\
\hline Diphoton vertex with largest $\Sigma p_{\mathrm{T}}^{2}$ & $<0.1-1.9$ & $<0.1-6.0$ & $\cdots$ \\
\hline Pileup reweighting & $0.2-5.6$ & $0.7-11$ & $\cdots$ \\
\hline Non-resonant background modeling & $\cdots$ & $\cdots$ & $0.1-9.8$ \\
\hline \multicolumn{4}{|l|}{ Theoretical } \\
\hline Factorization and renormalization scale & $0.6-11$ & $2.5-6.0$ & $\cdots$ \\
\hline $\mathrm{PDF}+\alpha_{\mathrm{S}}$ & $11-25$ & $1.2-2.9$ & $\cdots$ \\
\hline Multiple parton-parton interactions & $<1$ & $0.4-5.8$ & $\cdots$ \\
\hline $\mathcal{B}(H \rightarrow \gamma \gamma)$ & \multicolumn{2}{|c|}{1.73} & $\cdots$ \\
\hline
\end{tabular}


and its cluster is recalibrated as a photon cluster. The efficiency of this selection in data is found to be in agreement with the simulation within $0.01 \%$.

The systematic uncertainties due to the photon energy scale and resolution are adapted from run-1 results [41], with minor updates in case of data-driven corrections using the run-2 data. The uncertainty in the energy scale is less than $1 \%$ in the $p_{\mathrm{T}}$ range of the photons used in this analysis; the uncertainty in the energy resolution is smaller than $2 \%$.

Uncertainties in photon identification and isolation efficiencies are estimated, and their impact on the number of events in each category is quantified. The uncertainty in the photon identification efficiency [42] is calculated from the difference between applying and not applying the corrections to the electromagnetic-shower-shape variables in simulation. The resulting uncertainty in the photon identification efficiency is lower than $3.8 \%$ for SM Higgs background in all categories, 2.9\% for simplified model samples, and $4.3 \%$ for the heavy-scalar model. The uncertainty in the photon calorimeter isolation efficiency is calculated from efficiency differences between applying and not applying corrections derived from inclusive photon events to the isolation variables in simulation. The measurements of the efficiency correction factors using inclusive photon events are used to derive the efficiency uncertainty in the photon track isolation uncertainty. The photon isolation efficiency uncertainty is found to be smaller than $1.6 \%$ for the SM Higgs background and $1.2 \%$ for all signal samples.

Migration of events among categories occurs owing to changes in the energy of identified particles and jets, mostly due to the misreconstruction of jets and $E_{\mathrm{T}}^{\text {miss }}$. The uncertainties in jet energy scale, resolution, and jet vertex tagger are propagated to the $E_{\mathrm{T}}^{\text {miss }}$ calculation. In addition, the uncertainties in the scale and resolution of the $E_{\mathrm{T}}^{\text {miss }}$ soft term are estimated in 2016 data using the method described in Ref. [61]. The overall jet and $E_{\mathrm{T}}^{\text {miss }}$ uncertainties in the SM Higgs-boson processes are 6, 8, 23, 22, and $1 \%$ for the Mono-Higgs, high- $E_{\mathrm{T}}^{\text {miss }}$, intermediate- $E_{\mathrm{T}}^{\text {miss }}$, differentvertex, and rest categories, respectively. For signal processes, the overall jet and $E_{\mathrm{T}}^{\text {miss }}$ uncertainties range from $1.0 \%$ to $1.4 \%$. The choice of the diphoton vertex also affects the $E_{\mathrm{T}}^{\text {miss }}$ reconstruction. It introduces an additional uncertainty derived from the data-to-MC comparison in $Z \rightarrow e^{+} e^{-}$events. This systematic uncertainty affects the processes with no genuine $E_{\mathrm{T}}^{\text {miss }}$ and is estimated in each category. For the SM Higgs-boson production, it is found to be $0.5 \%$ in the Mono-Higgs category and up to $1.9 \%$ in the other categories. It is less than $0.1 \%$ for signal processes. Requiring the diphoton primary vertex to be the vertex with the largest $\Sigma p_{\mathrm{T}}^{2}$ in the event introduces an uncertainty of about $6 \%$ in the SM Higgs-boson production in the high$E_{\mathrm{T}}^{\text {miss }}$ and the intermediate- $E_{\mathrm{T}}^{\text {miss }}$ categories and $1.8 \%$ in the heavy-scalar signals in those categories. This systematic uncertainty differs between the signal and background because there is a correlation between the $E_{\mathrm{T}}^{\text {miss }}$ and the pileup vertex $\Sigma p_{T}^{2}$ for different processes. For other signal samples, these uncertainties are at most a few percent in any category. The pileup reweighting uncertainty is taken into account by propagating it through the event selection, and results in a $0.2 \%-5.6 \%$ uncertainty in the event yield of the signal and SM Higgs-boson samples.

The nonresonant background contribution is not affected by the same systematic uncertainties that characterize the MC simulation, since it is extracted from the data. The potential bias is added as a systematic uncertainty to account for the possible impact on the fitted signal yields of nonresonant background mismodeling. It is the leading systematic uncertainty of the analysis but is only one-third as large as the statistical uncertainty. The ratio of the potential bias in the signal yield to the expected nonresonant background $\left(\Delta N_{\mathrm{bkg}}^{\text {nonres }} / N_{\mathrm{bkg}}^{\text {nonres }}\right)$ in the range $120<$ $m_{\gamma \gamma}<130 \mathrm{GeV}$ is $9.8 \%$ in the Mono-Higgs category, $4.0 \%$ in the high- $E_{\mathrm{T}}^{\text {miss }}$ category, $1.3 \%$ in the intermediate- $E_{\mathrm{T}}^{\text {miss }}$ category, $0.5 \%$ in the different-vertex category, and $0.1 \%$ in the rest category.

The predicted cross sections of the SM Higgs-boson and signal processes are affected by uncertainties due to missing higher-order terms in perturbative QCD. These uncertainties are estimated by varying the factorization and renormalization scales up and down from their nominal values by a factor of 2 , recalculating the cross section in each case, and taking the largest deviation from the nominal cross section as the uncertainty. The uncertainty related to the renormalization and factorization scales is $0.6 \%-11 \%$ for signal and $2.5 \%-6.0 \%$ for the SM Higgs-boson processes [34]. For the three signal processes, the effect of $\mathrm{PDF}+\alpha_{\mathrm{S}}$ uncertainties including acceptance and normalization is $11 \%-25 \%$. It is estimated using the recommendations of PDF4LHC [34]. Both intra-PDF and inter-PDF uncertainties are extracted. Intra-PDF uncertainties are obtained by varying the parameters of the NNPDF3.0LO PDF set, while inter-PDF uncertainties are estimated using alternative PDF sets (CT14 [62] at LO and MMHT2014 [63] at LO). The final inter-PDF uncertainty is the maximum deviation among all the variations from the central value obtained using the NNPDF3.0LO PDF set. In the case of the SM Higgs-boson processes, the effect of $\alpha_{\mathrm{S}}$ and the choice of PDFs range from $2 \%$ to $6 \%$, which are taken from Ref. [34]. The uncertainty in the branching fraction $(\mathcal{B})$ of $h \rightarrow \gamma \gamma$ is $1.73 \%$ [34]. The uncertainty in the multiple parton-parton interactions is estimated by switching them on and off in PYTHIA 8.186 in the production of the ggF SM Higgs-boson sample. The resulting uncertainty in the number of events in this sample conservatively reaches $0.4 \%$ in the rest category, $5.8 \%$ in the differentvertex category, $3.8 \%$ intermediate- $E_{\mathrm{T}}^{\text {miss }}$ and differentvertex category, $3.4 \%$ in the high- $E_{\mathrm{T}}^{\text {miss }}$ category, and $1.4 \%$ in the Mono-Higgs category. 


\section{RESULTS}

The results for the analysis are derived from a likelihood fit of the $m_{\gamma \gamma}$ distribution in the range $105<m_{\gamma \gamma}<$ $160 \mathrm{GeV}$. The SM Higgs boson mass is set to $125.09 \mathrm{GeV}$ [35]. The impact due to the SM Higgs-boson mass uncertainty is negligible. The signal strength, the background shape parameters, and the nuisance parameters representing the systematic uncertainties are set to be free parameters. The SM Higgs yields are taken from the SM predictions. The systematic uncertainty of each nuisance parameter is taken into account by multiplying the likelihood by a Gaussian penalty function centered on the nominal value of this parameter with a width set to its uncertainty. The nominal value of each SM Higgs-boson background nuisance parameter (including its yield) is taken from the simulation normalized to the SM theoretical predictions. Since adding all the other categories to the Mono-Higgs category only brings a $1 \%$ sensitivity improvement for both the $Z_{B}^{\prime}$ and $Z^{\prime}-2 \mathrm{HDM}$ signals, the results are only obtained from this category for these two simplified models. In contrast, results for the heavy-scalar model are obtained from a simultaneous fit of all the categories.

The event yields in the observed data, expected signal and backgrounds in the five categories within a window of $120<m_{\gamma \gamma}<130 \mathrm{GeV}$ are shown in Table IV. The signal samples shown correspond to a $Z_{B}^{\prime}$ signal with $m_{Z_{B}^{\prime}}=$ $200 \mathrm{GeV}$ and $m_{\chi}=1 \mathrm{GeV}$, a $Z^{\prime}-2 \mathrm{HDM}$ signal with
$m_{Z^{\prime}}=1000 \mathrm{GeV}, m_{A^{0}}=200 \mathrm{GeV}$, and $m_{\chi}=100 \mathrm{GeV}$, and a heavy-scalar signal with $m_{H}=275 \mathrm{GeV}$ and $m_{\chi}=60 \mathrm{GeV}$. For each benchmark signal model, the selection efficiency times acceptance denoted by " $\mathcal{A} \times \epsilon$ " is also shown. The yields for the nonresonant background are obtained from a fit to data while SM Higgs-boson events are estimated from the simulation. The uncertainties correspond to the quadrature sum of the statistical and systematic uncertainties.

\section{A. Limits on the visible cross section}

The observed yields agree with the SM background predictions, as shown in Table IV, and no significant excess of events is observed. Upper limits are set on the visible cross section $\sigma_{\text {vis }}^{\mathrm{BSM}} \equiv(\mathcal{A} \times \epsilon \times \sigma \times \mathcal{B})^{\mathrm{BSM}}$ for BSM physics processes producing missing transverse momentum and a SM Higgs boson decaying into two photons. The SM background prediction is excluded from this BSM visible cross section. Table V shows the observed and expected 95\% confidence level (C.L.) upper limits on $\sigma_{\text {vis }}^{\mathrm{BSM}}$, which are calculated using a one-sided profile-likelihood ratio and the C.L.s formalism $[64,65]$ with the asymptotic approximation in Ref. [66]. The same parametrizations for the BSM signal and the total SM Higgs-boson background are used in each of the five different categories. The fits are performed individually in each category. The statistical uncertainty is dominant. The systematic uncertainties worsen the limits by about $10 \%$ (7\% from the nonresonant

TABLE IV. Event yields in the range of $120<m_{\gamma \gamma}<130 \mathrm{GeV}$ for data, signal models, the SM Higgs-boson background and nonresonant background in each analysis category, for an integrated luminosity of $36.1 \mathrm{fb}^{-1}$. The signal samples shown correspond to a heavy-scalar sample with $m_{H}=275 \mathrm{GeV}$ and $m_{\chi}=60 \mathrm{GeV}$, a $Z_{B}^{\prime}$ signal with $m_{Z_{B}^{\prime}}=200 \mathrm{GeV}$ and $m_{\chi}=1 \mathrm{GeV}$ and a $Z^{\prime}-2 \mathrm{HDM}$ signal with $m_{Z^{\prime}}=1000 \mathrm{GeV}, m_{A^{0}}=200 \mathrm{GeV}, m_{H^{0, \pm}}=300 \mathrm{GeV}$ and $m_{\chi}=100 \mathrm{GeV}$. For each benchmark signal model, the selection efficiency times acceptance denoted as " $\mathcal{A} \times \epsilon$ " is also shown. The yields for nonresonant background are obtained from a fit to data while SM Higgs-boson events are estimated from the simulation. The uncertainties correspond to the quadrature sum of the statistical and systematic uncertainties.

\begin{tabular}{|c|c|c|c|c|c|}
\hline Category & Mono-Higgs & High- $E_{\mathrm{T}}^{\mathrm{miss}}$ & Intermediate- $E_{\mathrm{T}}^{\text {miss }}$ & Different-vertex & Rest \\
\hline Data & 9 & 72 & 464 & 1511 & 46804 \\
\hline \multicolumn{6}{|c|}{ Backgrounds } \\
\hline SM Higgs boson & $2.43 \pm 0.22$ & $4.2 \pm 0.6$ & $11.9 \pm 2.7$ & $44 \pm 10$ & $1360 \pm 110$ \\
\hline Nonresonant & $9.9 \pm 1.9$ & $62 \pm 5$ & $418 \pm 10$ & $1490 \pm 18$ & $45570 \pm 110$ \\
\hline Total background & $12.3 \pm 1.9$ & $67 \pm 5$ & $430 \pm 10$ & $1535 \pm 21$ & $46930 \pm 170$ \\
\hline \multicolumn{6}{|c|}{$Z_{B}^{\prime}$ model, $m_{Z_{B}^{\prime}}=200 \mathrm{GeV}, m_{\chi}=1 \mathrm{GeV}$} \\
\hline Expected yields & $20.0 \pm 4.5$ & $\ldots$ & $\ldots$ & $\ldots$ & $\ldots$ \\
\hline $\mathcal{A} \times \epsilon[\%]$ & $17.4 \pm 0.2$ & $\cdots$ & $\cdots$ & $\cdots$ & $\cdots$ \\
\hline \multicolumn{6}{|c|}{$Z^{\prime}-2 \mathrm{HDM}$ model, $m_{Z^{\prime}}=1000 \mathrm{GeV}, m_{A^{0}}=200 \mathrm{GeV}, m_{H^{0, \pm}}=300 \mathrm{GeV}$, and $m_{\chi}=100 \mathrm{GeV}$} \\
\hline Expected yields & $28.0 \pm 5.3$ & $\ldots$ & $\ldots$ & $\ldots$ & $\cdots$ \\
\hline $\mathcal{A} \times \epsilon[\%]$ & $70.7 \pm 0.2$ & $\cdots$ & $\cdots$ & $\cdots$ & $\cdots$ \\
\hline \multicolumn{6}{|c|}{ Heavy-scalar model, $m_{H}=275 \mathrm{GeV}, m_{\chi}=60 \mathrm{GeV}$} \\
\hline Expected yields & $10.9 \pm 1.4$ & $23.8 \pm 3.2$ & $43 \pm 5$ & $33 \pm 5$ & $222 \pm 20$ \\
\hline $\mathcal{A} \times \epsilon[\%]$ & $1.22 \pm 0.07$ & $2.67 \pm 0.10$ & $4.82 \pm 0.14$ & $3.65 \pm 0.13$ & $24.9 \pm 0.4$ \\
\hline
\end{tabular}


TABLE V. Observed and expected upper limits (at 95\% C.L.) on the visible cross section for BSM physics processes producing missing transverse momentum and a SM Higgs boson decaying into two photons. Limits are presented for the five different categories. The $\pm 1 \sigma$ exclusion from the expected limits are also given. For all the simulated signal points, the lowest and largest values of the acceptance times efficiency $(\mathcal{A} \times \epsilon)$ for all three models are presented as a range. For the $Z_{B}^{\prime}$ model, signals with DM mass $m_{\chi}$ between 1 and $1000 \mathrm{GeV}$ and mediator mass $m_{Z_{B}^{\prime}}$ between 1 and $2000 \mathrm{GeV}$ are taken into consideration. The samples with the mediator mass $m_{Z^{\prime}}=400-1400 \mathrm{GeV}$ and pseudoscalar boson mass $m_{A^{0}}=200-450 \mathrm{GeV}$ are added for the $Z^{\prime}$-2HDM model. For the heavy-scalar model, the values are taken from the signals points with $m_{H}=260$ to $350 \mathrm{GeV}$ and $m_{\chi}=60 \mathrm{GeV}$.

\begin{tabular}{|c|c|c|c|c|c|}
\hline \multirow[b]{2}{*}{ Category } & \multicolumn{2}{|c|}{$\sigma_{\mathrm{vis}}^{\mathrm{BSM}}[\mathrm{fb}]$} & \multicolumn{3}{|c|}{$\mathcal{A} \times \epsilon[\%]$} \\
\hline & Observed & Expected & $Z^{\prime}-2 \mathrm{HDM}$ & $Z_{B}^{\prime}$ & Heavy scalar \\
\hline Mono-Higgs & 0.19 & $0.23_{-0.07}^{+0.11}$ & $53-74$ & $15-63$ & $1.0-4.0$ \\
\hline High- $E_{\mathrm{T}}^{\text {miss }}$ & 0.67 & $0.52_{-0.15}^{+0.23}$ & $0.2-12$ & $1.3-7.1$ & $1.8-8.4$ \\
\hline Intermediate- $E_{\mathrm{T}}^{\text {miss }}$ & 1.6 & $1.2_{-0.3}^{+0.5}$ & $0.05-5.0$ & $0.6-5.5$ & $3.9-6.6$ \\
\hline Different-vertex & 1.5 & $2.5_{-0.7}^{+1.1}$ & $0.04-11$ & $0.9-10$ & $2.5-7.4$ \\
\hline Rest & 11 & $15_{-4}^{+6}$ & $0.06-5.5$ & $1.1-22$ & $14-27$ \\
\hline
\end{tabular}

background modeling and 3\% from the other systematic uncertainties). The $\pm 1 \sigma$ variations from the expected limits are also given. For the Mono-Higgs category, visible cross sections $\sigma_{\text {vis }}^{\mathrm{BSM}}>0.19 \mathrm{fb}$ are excluded. The ranges of the acceptance times efficiency $(\mathcal{A} \times \epsilon)$ for all three different models considered in this paper are also shown. For the $Z_{B}^{\prime}$ model, signals with DM mass $m_{\chi}$ between 1 and $1000 \mathrm{GeV}$ and mediator mass $m_{Z_{B}^{\prime}}$ between 1 and $2000 \mathrm{GeV}$ are taken into consideration. The samples with the mediator mass

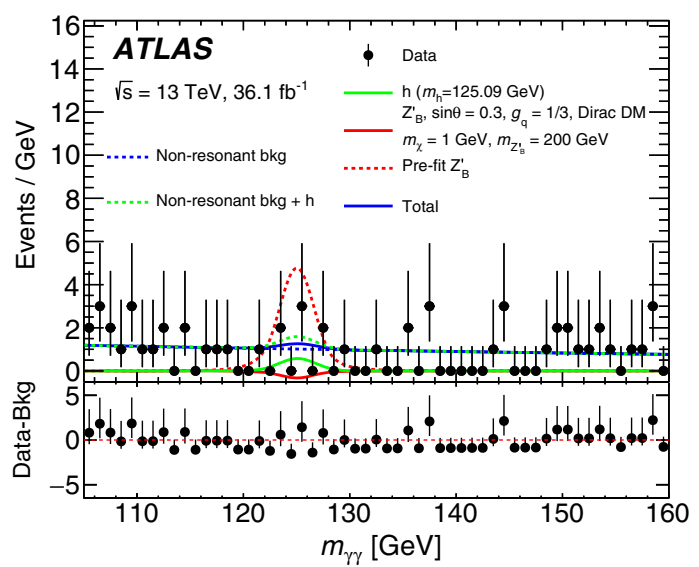

FIG. 3. Diphoton invariant mass distribution for data and the corresponding fitted signal and background in the Mono-Higgs category for the $Z_{B}^{\prime}$ benchmark model fit using $g_{q}=1 / 3, g_{\chi}=1$, $\sin \theta=0.3$, and Dirac fermion DM $m_{\chi}=1 \mathrm{GeV}$ as an illustration. A negative best-fit DM signal is found. The data is shown as dots with asymmetric error bars that represent central Poissonian confidence intervals at $68 \%$ C.L. The postfitted signal (solid red line), prefitted signal (dashed red line), SM Higgs boson (solid green line), nonresonant background (dashed blue line) and the nonresonant background plus the SM Higgs boson (dashed green line) are shown as well as the total of all those contributions (solid blue line). In the bottom panel, the "Bkg" represents the total background including the SM Higgs boson productions.
$m_{Z^{\prime}}=400-1400 \mathrm{GeV}$ and pseudoscalar boson mass $m_{A^{0}}=200-450 \mathrm{GeV}$ are added for the $Z^{\prime}-2$ HDM model. For the heavy-scalar model, the values are taken from the signals points with $m_{H}=260$ to $350 \mathrm{GeV}$ and $m_{\chi}=60 \mathrm{GeV}$.

\section{B. Interpretations of the $Z_{B}^{\prime}$ and $Z^{\prime}-2 H D M$ models}

Figure 3 shows the $m_{\gamma \gamma}$ distributions in the Mono-Higgs category as well as the fits for a $Z_{B}^{\prime}$ benchmark point with $m_{Z_{B}^{\prime}}=200 \mathrm{GeV}$ and $m_{\chi}=1 \mathrm{GeV}$. No significant excess is observed in this category. Upper limits are set on the production cross sections in the two theoretical models considered. Figure 4(a) shows the observed and median expected $95 \%$ C.L. upper limits on $\sigma(p p \rightarrow h \chi \bar{\chi}) \times$ $\mathcal{B}(h \rightarrow \gamma \gamma)$ as a function of the mediator mass $m_{Z_{B}^{\prime}}$ for a DM mass of $1 \mathrm{GeV}$. The cross sections times branching fraction of $h \rightarrow \gamma \gamma$ larger than $2.3 \mathrm{fb}$ are excluded for the full range of $m_{Z_{B}^{\prime}}$ between 10 and $2000 \mathrm{GeV}$ at $95 \%$ C.L., and the $Z_{B}^{\prime}$ model is excluded with $Z_{B}^{\prime}$ masses below $850 \mathrm{GeV}$ for a DM mass of $1 \mathrm{GeV}$.

In the $Z^{\prime}-2$ HDM scenario, the observed and median expected $95 \%$ C.L. upper limits on $\sigma(p p \rightarrow h \chi \bar{\chi}) \times$ $\mathcal{B}(h \rightarrow \gamma \gamma)$ are shown in Fig. 4(b), as a function of the pseudoscalar boson mass $m_{A^{0}}$ for $m_{\chi}=100 \mathrm{GeV}$ and $m_{Z^{\prime}}=1000 \mathrm{GeV}$. The masses of the neutral $C P$-even scalar $\left(H^{0}\right)$ and the charged scalars $\left(H^{ \pm}\right)$from $Z^{\prime}$-2HDM model are set to $300 \mathrm{GeV}$. The theoretical cross section starts from $m_{A^{0}}=201 \mathrm{GeV}$. The working point with $m_{A^{0}}=200 \mathrm{GeV}$ is excluded since the resonant production of DM particles at $m_{\chi}=100 \mathrm{GeV}$ significantly increases the cross section of the process. To avoid the resonant regime where $m_{A^{0}}=200 \mathrm{GeV}$ and $m_{\chi}=100 \mathrm{GeV}$ and allow a better limit interpolation, the point $m_{A^{0}}=201 \mathrm{GeV}$ is shown in this plot instead of $200 \mathrm{GeV}$. The drop of the theoretical prediction at $m_{A^{0}}=345 \mathrm{GeV}$ is due to a rapid change in the width when $A^{0}$ decaying to $t \bar{t}$ is kinematically 


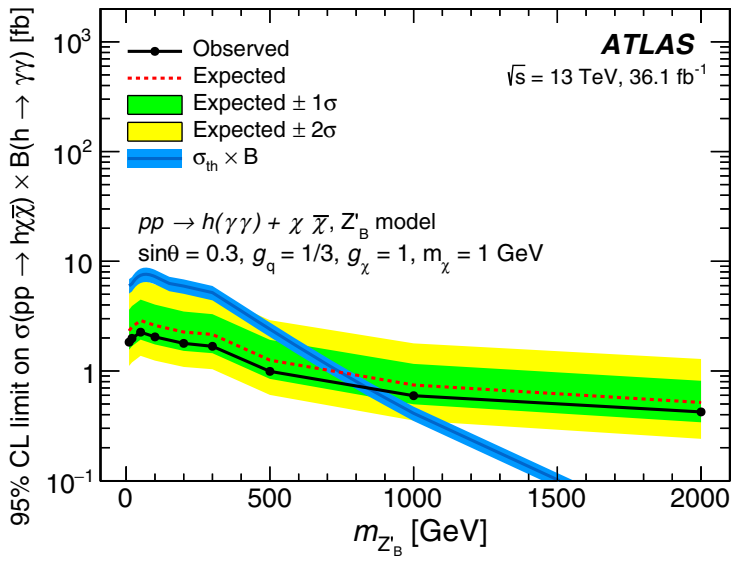

(a)

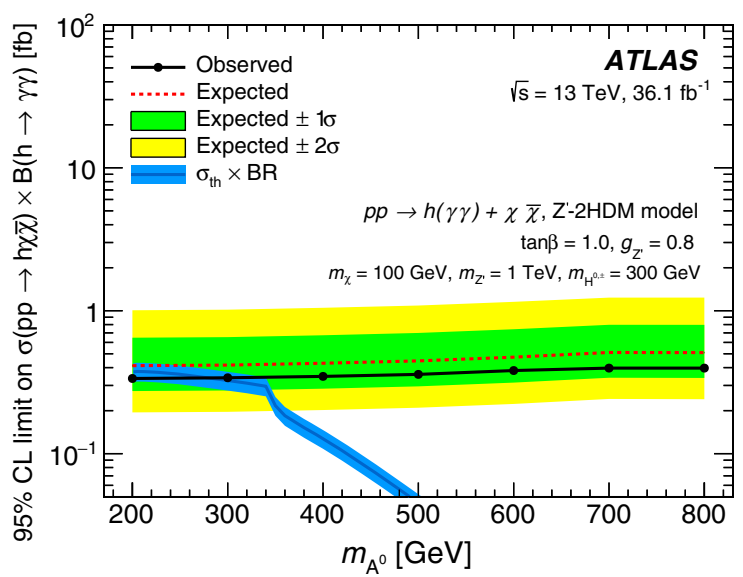

(b)

FIG. 4. Expected (dashed lines) and observed (solid lines) 95\% C.L. upper limits on $\sigma(p p \rightarrow h \chi \bar{\chi}) \times \mathcal{B}(h \rightarrow \gamma \gamma)$ for (a) the $Z_{B}^{\prime}$ model for $g_{q}=1 / 3, g_{\chi}=1, \sin \theta=0.3$ and Dirac fermion DM $m_{\chi}=1 \mathrm{GeV}$, and (b) the $Z^{\prime}-2 \mathrm{HDM}$ model for tan $\beta=1, g_{Z^{\prime}}=0.8, m_{Z^{\prime}}=$ $1000 \mathrm{GeV}$ and Dirac fermion DM $m_{\chi}=100 \mathrm{GeV}$, as a function of $m_{Z_{B}^{\prime}}$ and $m_{A^{0}}$, respectively. The masses of the neutral $C P$-even scalar $\left(H^{0}\right)$ and the charged scalars $\left(H^{ \pm}\right)$from $Z^{\prime}$-2HDM model are set to $300 \mathrm{GeV}$. The theoretical predictions of $\sigma(p p \rightarrow h \chi \bar{\chi}) \times \mathcal{B}(h \rightarrow \gamma \gamma)$ for these two models (dark-blue lines with blue bands representing their associated theoretical systematic uncertainties) are also shown. The inset shows a closeup view of the same figure in narrower ranges of both the $x$ and $y$ axes.

allowed. Pseudoscalar boson masses below $280 \mathrm{GeV}$ are excluded for $m_{Z^{\prime}}=1000 \mathrm{GeV}$ and $m_{\chi}=100 \mathrm{GeV}$. Tables VI and VII present the 95\% C.L. observed and median expected limits on $\sigma(p p \rightarrow h \chi \bar{\chi}) \times \mathcal{B}(h \rightarrow \gamma \gamma)$, respectively, for the $Z_{B}^{\prime}$ benchmark model for different $Z_{B}^{\prime}$ masses and the $Z^{\prime}$-2HDM model for different pseudoscalar $A^{0}$ masses. The corresponding expected limits with one standard deviation are also shown.

A two-dimensional exclusion region in the plane formed by the mediator masses and the DM particle mass is obtained by means of a reweighting technique based on generator-level variables. Samples for a variety of mass points are generated using the MADGRAPH generator matched to PYTHIA 8.186 using the A14 tune for parton showering and hadronization. Bin-by-bin weights for each of the different mass points are obtained by comparing the

TABLE VI. Observed and expected 95\% C.L. upper limits (in fb) on $\sigma(p p \rightarrow h \chi \bar{\chi}) \times \mathcal{B}(h \rightarrow \gamma \gamma)$ and associated expected $\pm 1 \sigma$ upper limits for the $Z_{B}^{\prime}$ benchmark model for different $m_{Z_{B}^{\prime}}$ and for a fixed mass $m_{\chi}=1 \mathrm{GeV}$.

\begin{tabular}{lcccc}
$\begin{array}{l}m_{Z_{B}^{\prime}} \\
{[\mathrm{GeV}]}\end{array}$ & Observed & Expected & Expected $+1 \sigma$ & Expected $-1 \sigma$ \\
\hline 10 & 1.83 & 2.33 & 3.61 & 1.56 \\
20 & 1.98 & 2.51 & 3.91 & 1.68 \\
50 & 2.26 & 2.88 & 4.47 & 1.93 \\
100 & 2.04 & 2.60 & 4.03 & 1.74 \\
200 & 1.78 & 2.26 & 3.48 & 1.52 \\
300 & 1.67 & 2.15 & 3.29 & 1.45 \\
500 & 0.99 & 1.25 & 1.92 & 0.85 \\
1000 & 0.59 & 0.74 & 1.16 & 0.50 \\
2000 & 0.42 & 0.51 & 0.81 & 0.34 \\
\hline \hline
\end{tabular}

generator level distribution of a given variable to the distribution for the same variable in a fully simulated benchmark sample. This procedure can be repeated for other variables. In the case of $Z_{B}^{\prime}$ samples, weights are derived using the true $E_{\mathrm{T}}^{\text {miss }}$. In the case of $Z^{\prime}-2 H D M$ samples, weights are derived in a two-dimensional plane of the true $E_{\mathrm{T}}^{\mathrm{miss}}$ and $p_{\mathrm{T}}^{\gamma \gamma}$. To validate this technique, several fully simulated and reconstructed samples are produced and their reconstruction-level variables are compared with the sample obtained from the reweighting technique. The acceptances of the samples after kinematic cuts agree within 5\%, and the residual difference is treated as an extra systematic uncertainty in the signal yield. The observed and expected 95\% C.L. limit contours for the signal strength $\sigma^{\text {obs }} / \sigma^{\text {th }}$ are shown in Fig. 5 for both the $Z_{B}^{\prime}$ and $Z^{\prime}$-2HDM models, in which $\sigma^{\text {obs }}$ is the observed limit on the model cross section at a given point of the parameter space and $\sigma^{\text {th }}$ is the predicted cross section in the model at the same point.

TABLE VII. Observed and expected 95\% C.L. upper limits (in fb) on $\sigma(p p \rightarrow h \chi \bar{\chi}) \times \mathcal{B}(h \rightarrow \gamma \gamma)$ and the associated expected $\pm 1 \sigma$ upper limits for the $Z^{\prime}-2 \mathrm{HDM}$ benchmark model for different $m_{A^{0}}$ and $m_{Z^{\prime}}=1000 \mathrm{GeV}, m_{\chi}=100 \mathrm{GeV}$.

$m_{A^{0}}$

$[\mathrm{GeV}]$ Observed Expected Expected $+1 \sigma \quad$ Expected $-1 \sigma$

\begin{tabular}{lllll}
\hline 200 & 0.33 & 0.41 & 0.65 & 0.27
\end{tabular}

$\begin{array}{lllll}300 & 0.34 & 0.42 & 0.65 & 0.28\end{array}$

$\begin{array}{lllll}400 & 0.35 & 0.43 & 0.67 & 0.28\end{array}$

$\begin{array}{lllll}500 & 0.38 & 0.45 & 0.70 & 0.30\end{array}$

$\begin{array}{lllll}600 & 0.39 & 0.47 & 0.74 & 0.31\end{array}$

$\begin{array}{lllll}700 & 0.40 & 0.51 & 0.80 & 0.34\end{array}$

$\begin{array}{lllll}800 & 0.40 & 0.51 & 0.80 & 0.34\end{array}$




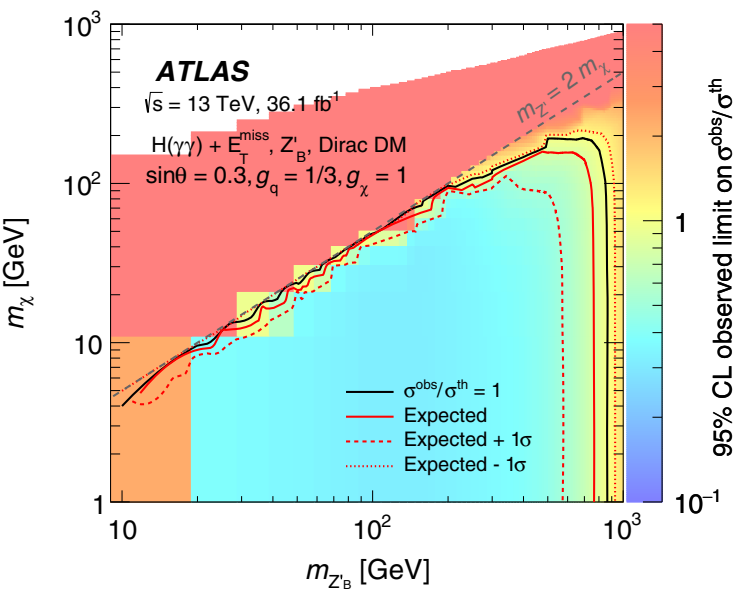

(a)

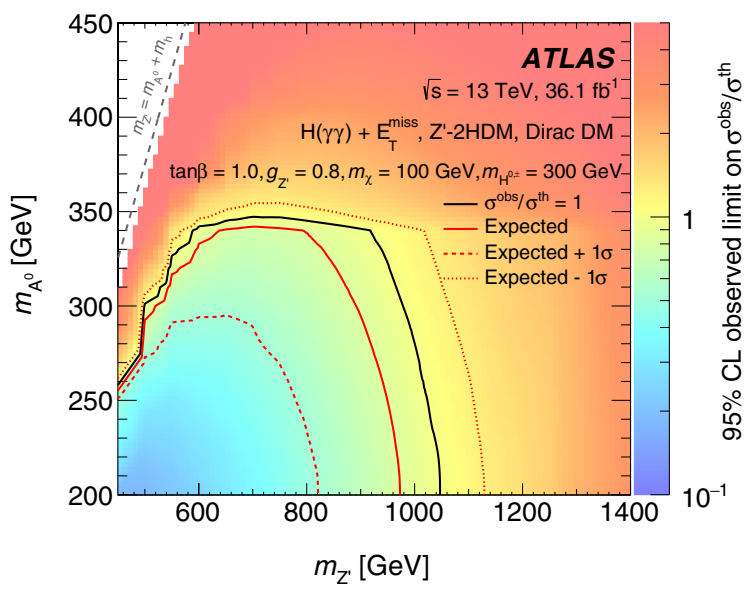

(b)

FIG. 5. The ratios of the observed and expected 95\% C.L. upper limits on the signal cross section to the predicted signal cross sections for (a) the $Z_{B}^{\prime}$ model in the $\left(m_{\chi}, m_{Z_{B}^{\prime}}\right)$ plane and (b) the $Z^{\prime}$-2HDM model in the $\left(m_{A^{0}}, m_{Z^{\prime}}\right)$ plane. For the $Z_{B}^{\prime}$ model, the mixing angle $\sin \theta=0.3$, and the coupling values $g_{q}=1 / 3$ and $g_{\chi}=1$ are used. In the scenario of $Z^{\prime}$-2HDM model, the ratio of the two-Higgs-doublet vacuum expectation values $\tan \beta=1.0$, Dirac fermion DM mass $m_{\chi}=100 \mathrm{GeV}$, and the coupling value $g_{Z^{\prime}}=0.8$ are used. The masses of the neutral $C P$-even scalar $\left(H^{0}\right)$ and the charged scalars $\left(H^{ \pm}\right)$from $Z^{\prime}$-2HDM model are set to $300 \mathrm{GeV}$. The plus and minus one standard deviation expected exclusion curves are also shown as red dashed and dotted lines. The regions below the lines (i.e. with $\sigma^{\text {obs }} / \sigma^{\text {th }}<1$ ) are excluded. In both figures, the gray dashed line corresponds to the boundary of the region above which the $Z^{\prime}$ boson is produced off shell.

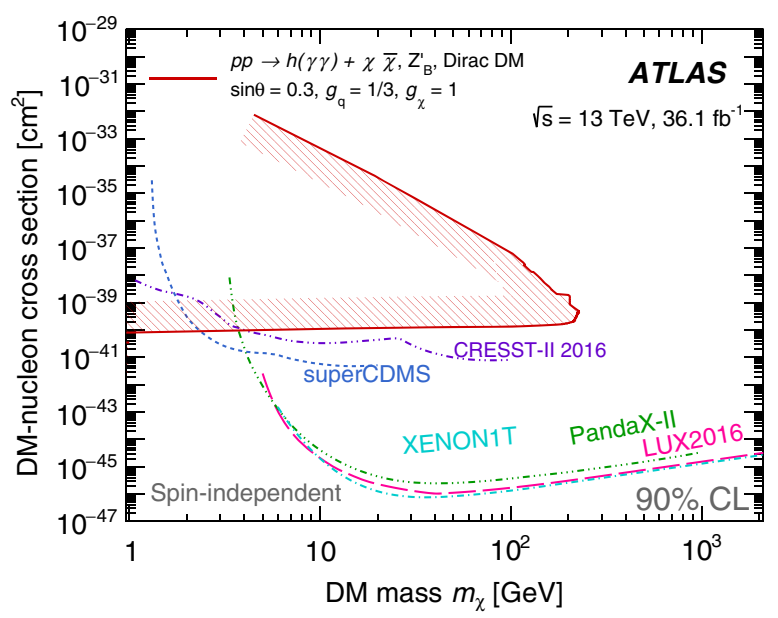

FIG. 6. A comparison of the inferred limits to the constraints from direct detection experiments on the spin-independent DM-nucleon cross section in the context of the $Z_{B}^{\prime}$ simplified model with vector couplings. Limits are shown at 90\% C.L. The results from this analysis, in which the region inside the contour is excluded, are compared with limits from the LUX [67], PandaX-II [68], XENON [69], superCDMS [70], and CRESST-II [71] experiments. The comparison is model dependent and solely valid in the context of this model, assuming Dirac fermion DM, mixing angle $\sin \theta=0.3$, and the coupling values $g_{q}=1 / 3$ and $g_{\chi}=1$. The impact of renormalization-group evolution effects $[72,73]$ when comparing collider and direct detection limits is not taken into consideration here.
Figure 6 shows a comparison of the inferred limits to the constraints from direct detection experiments on the spinindependent (SI) DM-nucleon cross section in the context of the $Z_{B}^{\prime}$ simplified model with vector couplings using the relation [5]

$$
\sigma_{N \chi}^{\mathrm{SI}}=\frac{\mu_{N \chi}^{2}}{\pi A^{2}}\left[Z f_{p}-(A-Z) f_{n}\right]^{2},
$$

in which $\mu_{N \chi}=m_{\chi} m_{N} /\left(m_{\chi}+m_{N}\right)$ is the reduced mass of the DM-nucleon system, and $f_{p, n}=3 g_{q} g_{\chi} / m_{Z_{B}^{\prime}}^{2}$ are the couplings between DM particles and protons and neutrons. The parameter $Z$ is the number of protons in the considered nucleus and $A$ the number of nucleons (both set to 1 ). Limits are shown at $90 \%$ C.L. For comparison, results from direct detection experiments (LUX [67], PandaX-II [68], XENON [69], superCDMS [70], and CRESST-II [71]) are also shown. The comparison is model dependent and solely valid in the context of this model. The results for the $Z_{B}^{\prime}$ model, with couplings $g_{q}=1 / 3$ and $g_{\chi}=1$ for this search, are more stringent than direct detection experiments for $m_{\chi}<2.5 \mathrm{GeV}$ and extend to DM masses well below $1 \mathrm{GeV}$. The shape of the exclusion line at DM mass $m_{\chi} \sim$ $200 \mathrm{GeV}$ to low masses is due to the loss of sensitivity in $Z_{B}^{\prime}$ models where DM particles are produced via an offshell process. The impact of renormalization-group evolution effects [72,73] when comparing collider and direct detection limits is not taken into consideration here. 


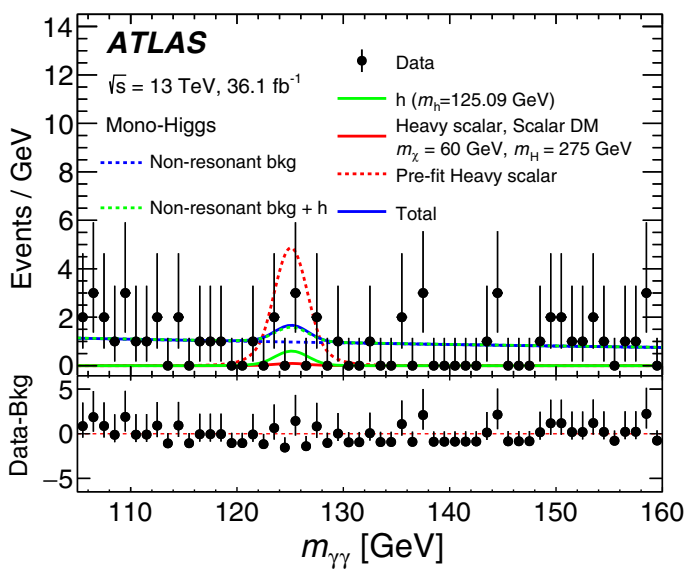

(a)

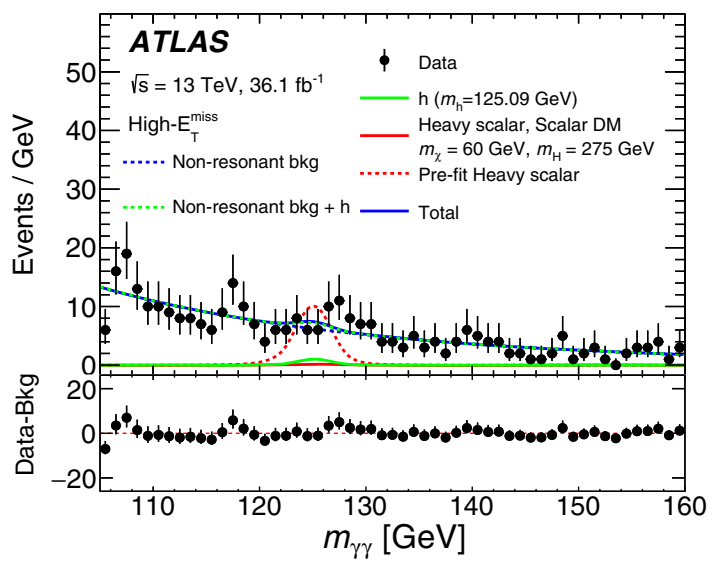

(b)

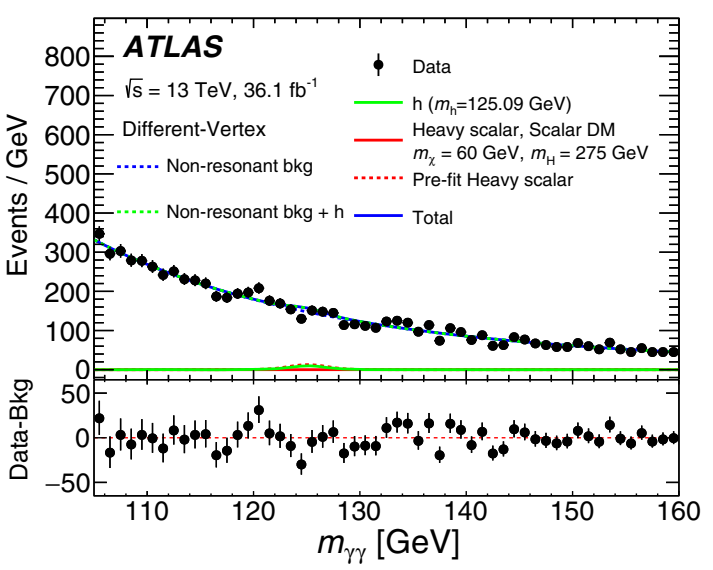

(d)

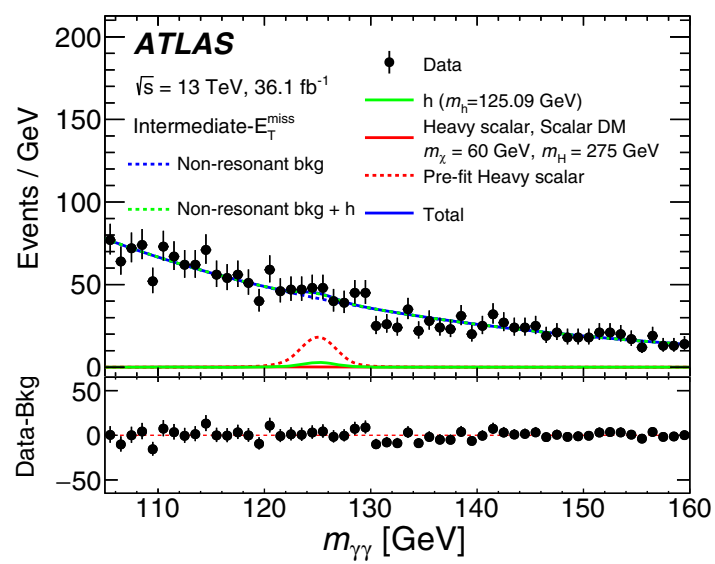

(c)

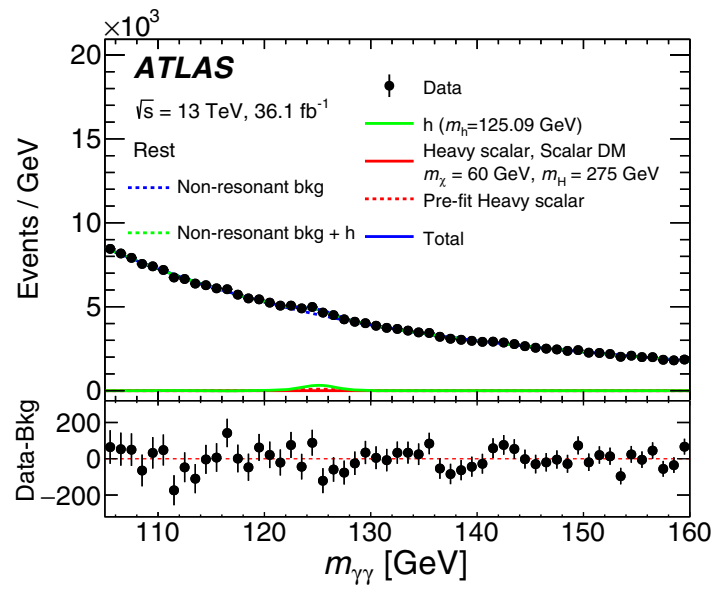

(e)

FIG. 7. Diphoton invariant mass distribution for data and the corresponding fitted signal and background in the five categories, (a) Mono-Higgs category, (b) high- $E_{\mathrm{T}}^{\text {miss }}$ category, (c) intermediate- $E_{\mathrm{T}}^{\text {miss }}$ category, (d) different-vertex category, and (e) rest category. In each plot, the data (dots with asymmetric error bars) is shown. The error bars represent the central Poissonian confidence intervals at $68 \%$ C.L. The simultaneous fit result including a heavy-scalar signal (solid red line), SM Higgs boson (solid green line), the nonresonant background (dashed blue line), and the nonresonant background plus the SM Higgs boson (dashed green line) are shown as well as the sum of all those contributions (solid blue line). In the bottom panel, the "Bkg" represents the total background including the SM Higgs boson productions. The both prefitted and postfitted heavy-scalar signals shown here correspond to $m_{H}=275 \mathrm{GeV}$ and scalar DM $m_{\chi}=60 \mathrm{GeV}$. 


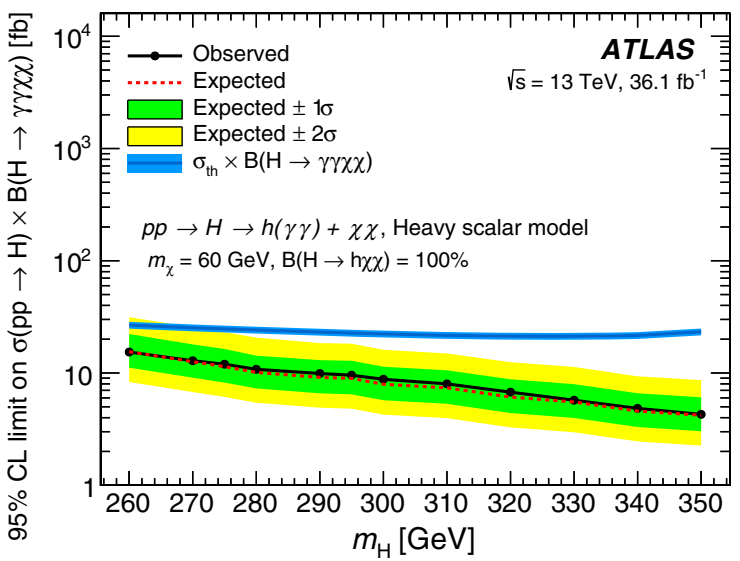

FIG. 8. Observed and expected $95 \%$ C.L. upper limits on the $\sigma(p p \rightarrow H) \times \mathcal{B}(H \rightarrow \gamma \gamma \chi \chi)$ with a scalar DM candidate mass of $60 \mathrm{GeV}$ as a function of the heavy-scalar-boson mass in the range $260<m_{H}<350 \mathrm{GeV}$. A $100 \%$ branching fraction is assumed for $H \rightarrow h \chi \chi$. The theoretical prediction for the model (dark blue lines with blue bands representing their associated theoretical systematic uncertainties) is also shown. The theoretical cross section is assumed to be equal to that of a SM Higgs boson with the same mass produced in gluon-gluon fusion.

\section{Interpretation of the heavy-scalar model}

Figure 7 shows the $m_{\gamma \gamma}$ distributions in the five categories as well as the fitted contribution of a heavy-scalar boson for illustration. No significant excess is observed in any category. In the heavy-scalar interpretation, the 95\% C.L. upper limits on the $\sigma(p p \rightarrow H) \times \mathcal{B}(H \rightarrow \gamma \gamma \chi \chi)$ as a function of $m_{H}$ for $m_{\chi}=60 \mathrm{GeV}$ are shown in Fig. 8 and Table VIII, where a $100 \%$ branching fraction is assumed for $H \rightarrow h \chi \chi$. The upper limit at $95 \%$ C.L. is $15.4 \mathrm{fb}$ for $m_{H}=260 \mathrm{GeV}$, and $4.3 \mathrm{fb}$ for $m_{H}=350 \mathrm{GeV}$.

TABLE VIII. Observed and expected 95\% C.L. upper limits (in fb) on the $\sigma(p p \rightarrow H) \times \mathcal{B}(H \rightarrow \gamma \gamma \chi \chi)$, where a $100 \%$ branching fraction is assumed for $H \rightarrow h \chi \chi$, and the associated expected $\pm 1 \sigma$ upper limits for the heavy-scalar model for different mediator masses and $m_{\chi}=60 \mathrm{GeV}$.

\begin{tabular}{lcccc}
\hline \hline$m_{H}[\mathrm{GeV}]$ & Observed & Expected & Expected $+1 \sigma$ & Expected $-1 \sigma$ \\
\hline 260 & 15.4 & 15.5 & 22.3 & 11.2 \\
270 & 12.9 & 12.6 & 18.0 & 9.1 \\
275 & 12.0 & 11.4 & 16.4 & 8.2 \\
285 & 10.8 & 11.1 & 14.3 & 7.3 \\
290 & 9.9 & 9.1 & 13.0 & 6.6 \\
295 & 9.6 & 9.0 & 12.8 & 6.5 \\
300 & 8.8 & 7.9 & 11.4 & 5.7 \\
310 & 8.0 & 7.4 & 10.6 & 5.3 \\
320 & 6.8 & 6.1 & 8.8 & 4.4 \\
330 & 5.7 & 5.5 & 7.9 & 4.0 \\
340 & 4.8 & 4.6 & 6.6 & 3.3 \\
350 & 4.3 & 4.2 & 6.1 & 3.0 \\
\hline \hline
\end{tabular}

\section{SUMMARY}

A search for dark matter in association with a Higgs boson decaying to two photons is presented. This study is based on data collected with the ATLAS detector, corresponding to an integrated luminosity of $36.1 \mathrm{fb}^{-1}$ of proton-proton collisions at the LHC at a center-of-mass energy of $13 \mathrm{TeV}$ in 2015 and 2016. No significant excess over the expected background is observed. For the MonoHiggs category, a visible cross section larger than $0.19 \mathrm{fb}$ is excluded at $95 \%$ C.L. for BSM physics processes producing missing transverse momentum and a SM Higgs boson decaying into two photons. Upper limits at $95 \%$ C.L. are also set on the production cross section times branching fraction of the Higgs boson decaying into two photons in association with missing transverse momentum in three different benchmark models: a $Z_{B}^{\prime}$ model, a $Z^{\prime}-2 \mathrm{HDM}$ model and a heavy scalar boson $(H)$ model. Limits at 95\% C.L. are also set on the observed signal strength in a two-dimensional $m_{\chi}-m_{Z_{B}^{\prime}}$ plane for the $Z_{B}^{\prime}$ model, and the $m_{A^{0}}-m_{Z^{\prime}}$ plane for the $Z^{\prime}$-2HDM model. Additionally, the results for the $Z_{B}^{\prime}$ model are interpreted in terms of $90 \%$ C.L. limits on the dark-matter-nucleon scattering cross section, as a function of the dark-matterparticle mass, for a spin-independent scenario. For a dark-matter mass lower than $2.5 \mathrm{GeV}$, the constraint with couplings $g_{q}=1 / 3$ and $g_{\chi}=1$ placed on the DMnucleon cross section is more stringent than limits from direct detection experiments. In the model involving the production of a heavy scalar boson, 95\% C.L. upper limits are set on the production cross section times the branching fraction of $H \rightarrow h \chi \chi \rightarrow \gamma \gamma \chi \chi$ for a dark-matter particle with mass of $60 \mathrm{GeV}$, where a $100 \%$ branching fraction is assumed for $H \rightarrow h \chi \chi$. The heavy-scalar model assuming $H$ production through gluon-gluon fusion with a cross section identical to that of a SM Higgs boson of the same mass, is excluded for all the benchmark points investigated.

\section{ACKNOWLEDGMENTS}

We thank CERN for the very successful operation of the LHC, as well as the support staff from our institutions without whom ATLAS could not be operated efficiently. We acknowledge the support of ANPCyT, Argentina; YerPhI, Armenia; ARC, Australia; BMWFW and FWF, Austria; ANAS, Azerbaijan; SSTC, Belarus; CNPq and FAPESP, Brazil; NSERC, NRC and CFI, Canada; CERN; CONICYT, Chile; CAS, MOST and NSFC, China; COLCIENCIAS, Colombia; MSMT CR, MPO CR and VSC CR, Czech Republic; DNRF and DNSRC, Denmark; IN2P3-CNRS, CEA-DSM/IRFU, France; SRNSF, Georgia; BMBF, HGF, and MPG, Germany; GSRT, Greece; RGC, Hong Kong SAR, China; ISF, I-CORE and Benoziyo Center, Israel; INFN, Italy; MEXT and JSPS, Japan; CNRST, Morocco; NWO, Netherlands; 
RCN, Norway; MNiSW and NCN, Poland; FCT, Portugal; MNE/IFA, Romania; MES of Russia and NRC KI, Russian Federation; JINR; MESTD, Serbia; MSSR, Slovakia; ARRS and MIZŠ, Slovenia; DST/NRF, South Africa; MINECO, Spain; SRC and Wallenberg Foundation, Sweden; SERI, SNSF and Cantons of Bern and Geneva, Switzerland; MOST, Taiwan; TAEK, Turkey; STFC, United Kingdom; DOE and NSF, United States of America. In addition, individual groups and members have received support from BCKDF, the Canada Council, CANARIE, CRC, Compute Canada, FQRNT, and the Ontario Innovation Trust, Canada; EPLANET, ERC, ERDF, FP7, Horizon 2020 and Marie Skłodowska-Curie Actions, European Union; Investissements d'Avenir Labex and Idex, ANR, Région Auvergne and Fondation Partager le Savoir, France; DFG and AvH Foundation, Germany; Herakleitos, Thales and Aristeia programmes co-financed by EU-ESF and the Greek NSRF; BSF, GIF and Minerva, Israel; BRF, Norway; CERCA Programme Generalitat de Catalunya, Generalitat Valenciana, Spain; the Royal Society and Leverhulme Trust, United Kingdom. The crucial computing support from all WLCG partners is acknowledged gratefully, in particular from CERN, the ATLAS Tier-1 facilities at TRIUMF (Canada), NDGF (Denmark, Norway, Sweden), CC-IN2P3 (France), KIT/ GridKA (Germany), INFN-CNAF (Italy), NL-T1 (Netherlands), PIC (Spain), ASGC (Taiwan), RAL (UK) and BNL (USA), the Tier-2 facilities worldwide and large non-WLCG resource providers. Major contributors of computing resources are listed in Ref. [74].
[1] ATLAS Collaboration, Observation of a new particle in the search for the Standard Model Higgs boson with the ATLAS detector at the LHC, Phys. Lett. B 716, 1 (2012).

[2] CMS Collaboration, Observation of a new boson at a mass of $125 \mathrm{GeV}$ with the CMS experiment at the LHC, Phys. Lett. B 716, 30 (2012).

[3] C. Patrignani et al., Review of Particle Physics, Chin. Phys. C 40, 100001 (2016).

[4] G. Bertone, D. Hooper, and J. Silk, Particle dark matter: evidence, candidates and constraints, Phys. Rep. 405, 279 (2005).

[5] L. Carpenter, A. DiFranzo, M. Mulhearn, C. Shimmin, S. Tulin, and D. Whiteson, Mono-Higgs-boson: A new collider probe of dark matter, Phys. Rev. D 89, 075017 (2014).

[6] A. A. Petrov and W. Shepherd, Searching for dark matter at LHC with Mono-Higgs production, Phys. Lett. B 730, 178 (2014).

[7] ATLAS Collaboration, Search for Dark Matter in Events with Missing Transverse Momentum and a Higgs Boson Decaying to Two Photons in $p p$ Collisions at $\sqrt{s}=8 \mathrm{TeV}$ with the ATLAS Detector, Phys. Rev. Lett. 115, 131801 (2015).

[8] ATLAS Collaboration, Search for dark matter produced in association with a Higgs boson decaying to two bottom quarks in $p p$ collisions at $\sqrt{s}=8 \mathrm{TeV}$ with the ATLAS detector, Phys. Rev. D 93, 072007 (2016).

[9] ATLAS Collaboration, Search for dark matter in association with a Higgs boson decaying to $b$-quarks in $p p$ collisions at $\sqrt{s}=13 \mathrm{TeV}$ with the ATLAS detector, Phys. Lett. B 765, 11 (2017).

[10] CMS Collaboration, Search for associated production of dark matter with a Higgs boson decaying to $b \bar{b}$ or $\gamma \gamma$ at $\sqrt{s}=13 \mathrm{TeV}$, arXiv:1703.05236.

[11] ATLAS Collaboration, Search for Dark Matter Produced in Association with a Higgs Boson Decaying to $b \bar{b}$ using $36 \mathrm{fb}^{-1}$ of $p$ Collisions at $\sqrt{s}=13 \mathrm{TeV}$ with the ATLAS Detector, Phys. Rev. Lett. 119, 181804 (2017).
[12] D. Abercrombie et al., Dark matter benchmark models for early LHC run-2 searches: Report of the ATLAS/CMS dark matter forum, arXiv:1507.00966.

[13] A. Berlin, T. Lin, and L.-T. Wang, Mono-Higgs detection of dark matter at the LHC, J. High Energy Phys. 06 (2014) 078 .

[14] S. von Buddenbrock, N. Chakrabarty, A. S. Cornell, D. Kar, M. Kumar, T. Mandal, B. Mellado, B. Mukhopadhyaya, R. G. Reed, and X. Ruan, Phenomenological signatures of additional scalar bosons at the LHC, Eur. Phys. J. C 76, 580 (2016).

[15] W.-L. Guo and Y.-L. Wu, The real singlet scalar dark matter model, J. High Energy Phys. 10 (2010), 083.

[16] ATLAS Collaboration, The ATLAS experiment at the CERN Large Hadron Collider, J. Instrum. 3, S08003 (2008).

[17] ATLAS Collaboration, Report No. ATLAS-TDR-19, https:// cds.cern.ch/record/1291633; Report No. ATLAS-TDR-023, https://cds.cern.ch/record/1602235.

[18] ATLAS Collaboration, Performance of the ATLAS Trigger System in 2015, Eur. Phys. J. C 77, 317 (2017).

[19] ATLAS Collaboration, Search for resonances in diphoton events at $\sqrt{s}=13 \mathrm{TeV}$ with the ATLAS detector, J. High Energy Phys. 09 (2016) 001.

[20] J. Alwall, R. Frederix, S. Frixione, V. Hirschi, F. Maltoni, O. Mattelaer, H.-S. Shao, T. Stelzer, P. Torrielli, and M. Zaro, The automated computation of tree-level and next-toleading order differential cross sections, and their matching to parton shower simulations, J. High Energy Phys. 07 (2014) 79.

[21] R. D. Ball et al., Parton distributions for the LHC run II, J. High Energy Phys. 04 (2015) 040.

[22] T. Sjostrand, S. Mrenna, and P. Skands, A brief introduction to PYTHIA 8.1, Comput. Phys. Commun. 178, 852 (2008).

[23] ATLAS Collaboration, Report No. ATL-PHYS-PUB-2014021, https://cds.cern.ch/record/1966419.

[24] R. D. Ball et al. (NNPDF Collaboration), Parton distributions with LHC data, Nucl. Phys. B867, 244 (2013). 
[25] P. Nason, A new method for combining NLO QCD with shower Monte Carlo algorithms, J. High Energy Phys. 11 (2004) 040.

[26] S. Alioli, P. Nason, C. Oleari, and E. Re, NLO Higgs boson production via gluon fusion matched with shower in POWHEG, J. High Energy Phys. 04 (2009) 002.

[27] P. Nason and C. Oleari, NLO Higgs boson production via vector-boson fusion matched with shower in POWHEG, J. High Energy Phys. 02 (2010) 037.

[28] S. Alioli, P. Nason, C. Oleari, and E. Re, A general framework for implementing NLO calculations in shower Monte Carlo programs: The POWHEG BOX, J. High Energy Phys. 06 (2010) 043.

[29] ATLAS Collaboration, Report No. ATL-PHYS-PUB-2013017, https://cds.cern.ch/record/1629317.

[30] H.-L. Lai, M. Guzzi, J. Huston, Z. Li, P. M. Nadolsky, J. Pumplin, and C.-P. Yuan, New parton distributions for collider physics, Phys. Rev. D 82, 074024 (2010).

[31] T. Gleisberg et al., Event generation with SHERPA 1.1, J. High Energy Phys. 02 (2009) 007.

[32] S. Schumann and F. Krauss, A Parton shower algorithm based on Catani-Seymour dipole factorisation, J. High Energy Phys. 03 (2008) 038.

[33] S. Höche, F. Krauss, S. Schumann, and F. Siegert, QCD matrix elements and truncated showers, J. High Energy Phys. 05 (2009) 053.

[34] D. de Florian et al., Handbook of LHC Higgs cross sections: 4. Deciphering the nature of the Higgs sector, arXiv:1610.07922.

[35] ATLAS and CMS Collaborations, Combined Measurement of the Higgs Boson Mass in $p p$ Collisions at $\sqrt{s}=7$ and $8 \mathrm{TeV}$ with the ATLAS and CMS Experiments, Phys. Rev. Lett. 114, 191803 (2015).

[36] D. J. Lange, The EvtGen particle decay simulation package, Nucl. Instrum. Methods Phys. Res., Sect. A 462, 152 (2001).

[37] ATLAS Collaboration, Report No. ATL-PHYS-PUB-2012003, https://cds.cern.ch/record/1474107/.

[38] ATLAS Collaboration, The ATLAS Simulation Infrastructure, Eur. Phys. J. C 70, 823 (2010).

[39] S. Agostinelli et al., GEANT4-A Simulation toolkit, Nucl. Instrum. Methods Phys. Res., Sect. A 506, 250 (2003).

[40] ATLAS Collaboration, Measurement of the photon identification efficiencies with the ATLAS detector using LHC Run-1 data, Eur. Phys. J. C 76, 666 (2016).

[41] ATLAS Collaboration, Electron and photon energy calibration with the ATLAS detector using LHC Run 1 data, Eur. Phys. J. C 74, 3071 (2014).

[42] ATLAS Collaboration, Report No. ATL-PHYS-PUB-2016014, https://cds.cern.ch/record/2203125.

[43] ATLAS Collaboration, Measurement of Higgs boson production in the diphoton decay channel in pp collisions at center-of-mass energies of 7 and $8 \mathrm{TeV}$ with the ATLAS detector, Phys. Rev. D 90, 112015 (2014).

[44] ATLAS Collaboration, Topological cell clustering in the ATLAS calorimeters and its performance in LHC Run 1, Eur. Phys. J. C 77, 490 (2017).

[45] M. Cacciari, G. P. Salam, and G. Soyez, The catchment area of jets, J. High Energy Phys. 04 (2008) 005.
[46] ATLAS Collaboration, Performance of pile-up mitigation techniques for jets in $p p$ collisions at $\sqrt{s}=8 \mathrm{TeV}$ using the ATLAS detector, Eur. Phys. J. C 76, 581 (2016).

[47] ATLAS Collaboration, Measurement of the Higgs boson mass from the $H \rightarrow \gamma \gamma$ and $H \rightarrow Z Z^{*} \rightarrow 4 \ell$ channels with the ATLAS detector, Phys. Rev. D 90, 052004 (2014).

[48] ATLAS Collaboration, Electron efficiency measurements with the ATLAS detector using 2012 LHC proton-proton collision data, Eur. Phys. J. C 77, 195 (2017).

[49] ATLAS Collaboration, Report No. ATL-PHYS-PUB-2015041, https://cds.cern.ch/record/2048202.

[50] ATLAS Collaboration, Measurements of fiducial and differential cross sections for Higgs boson production in the diphoton decay channel at $\sqrt{s}=8 \mathrm{TeV}$ with ATLAS, J. High Energy Phys. 09 (2014) 112.

[51] ATLAS Collaboration, Measurement of the muon reconstruction performance of the ATLAS detector using 2011 and 2012 LHC proton-proton collision data, Eur. Phys. J. C 74, 3130 (2014).

[52] ATLAS Collaboration, Muon reconstruction performance of the ATLAS detector in proton-proton collision data at $\sqrt{s}=13 \mathrm{TeV}$, Eur. Phys. J. C 76, 292 (2016).

[53] M. Cacciari, G. P. Salam, and G. Soyez, The anti-kt jet clustering algorithm, J. High Energy Phys. 04 (2008) 063.

[54] ATLAS Collaboration, Report No. ATL-PHYS-PUB-2014001, https://cds.cern.ch/record/1643929.

[55] ATLAS Collaboration, Jet energy scale measurements and their systematic uncertainties in proton-proton collisions at $\sqrt{s}=13 \mathrm{TeV}$ with the ATLAS detector, Phys. Rev. D 96, 072002 (2017).

[56] ATLAS Collaboration, Performance of missing transverse momentum reconstruction in proton-proton collisions at $\sqrt{s}=7 \mathrm{TeV}$ with ATLAS, Eur. Phys. J. C 72, 1844 (2012).

[57] ATLAS Collaboration, Report No. ATL-PHYS-PUB-2015027, https://cds.cern.ch/record/2037904.

[58] ATLAS Collaboration, Measurement of isolated-photon pair production in $p p$ collisions at $\sqrt{s}=7 \mathrm{TeV}$ with the ATLAS detector, J. High Energy Phys. 01 (2013) 086.

[59] T. Aaltonen et al. (CDF Collaboration), Search for new particles decaying into dijets in proton-antiproton collisions at $\sqrt{s}=1.96 \mathrm{TeV}$, Phys. Rev. D 79, 112002 (2009).

[60] ATLAS Collaboration, Luminosity determination in pp collisions at $\sqrt{s}=8 \mathrm{TeV}$ using the ATLAS detector at the LHC, Eur. Phys. J. C 76, 653 (2016).

[61] ATLAS Collaboration, Report No. ATL-PHYS-PUB-2015023, https://cds.cern.ch/record/2037700.

[62] S. Dulat, T.-J. Hou, J. Gao, M. Guzzi, J. Huston, P. Nadolsky, J. Pumplin, C. Schmidt, D. Stump, and C.-P. Yuan, New parton distribution functions from a global analysis of quantum chromodynamics, Phys. Rev. D 93, 033006 (2016).

[63] L. A. Harland-Lang, A. D. Martin, P. Motylinski, and R. S. Thorne, Parton distributions in the LHC era: MMHT 2014 PDFs, Eur. Phys. J. C 75, 204 (2015).

[64] A. L. Read, Presentation of search results: The CLs technique, J. Phys. G 28, 2693 (2002).

[65] T. Junk, Confidence level computation for combining searches with small statistics, Nucl. Instrum. Methods Phys. Res., Sect. A 434, 435 (1999). 
[66] G. Cowan, K. Cranmer, E. Gross, and O. Vitells, Asymptotic formulae for likelihood-based tests of new physics, Eur. Phys. J. C 71, 1554 (2011); Erratum, Eur. Phys. J. C 73, 2501 (2013).

[67] D. S. Akerib et al. (LUX Collaboration), Results from a Search for Dark Matter in the Complete LUX exposure, Phys. Rev. Lett. 118, 021303 (2017).

[68] A. Tan et al. (PandaX-II Collaboration), Dark Matter Results from First 98.7 Days of Data from the PandaX-II Experiment, Phys. Rev. Lett. 117, 121303 (2016).

[69] E. Aprile et al. (XENON Collaboration), First Dark Matter Search Results from the XENON1T Experiment, Phys. Rev. Lett. 119, 181301 (2017).

[70] R. Agnese et al. (SuperCDMS Collaboration), New Results from the Search for Low-Mass Weakly Interacting
Massive Particles with the CDMS Low Ionization Threshold Experiment, Phys. Rev. Lett. 116, 071301 (2016).

[71] G. Angloher et al. (CRESST Collaboration), Results on light dark matter particles with a low-threshold CRESST-II detector, Eur. Phys. J. C 76, 25 (2016).

[72] A. Crivellin, F. D’Eramo, and M. Procura, New Constraints on Dark Matter Effective Theories from Standard Model Loops, Phys. Rev. Lett. 112, 191304 (2014).

[73] F. D'Eramo and M. Procura, Connecting dark matter UV complete models to direct detection rates via effective field theory, J. High Energy Phys. 04 (2015) 054.

[74] ATLAS Collaboration, Report No. ATL-GEN-PUB-2016002, https://cds.cern.ch/record/2202407.

M. Aaboud, ${ }^{137 d}$ G. Aad ${ }^{88}$ B. Abbott, ${ }^{115}$ O. Abdinov, ${ }^{12, a}$ B. Abeloos, ${ }^{119}$ S. H. Abidi, ${ }^{161}$ O. S. AbouZeid, ${ }^{139}$ N. L. Abraham, ${ }^{151}$ H. Abramowicz, ${ }^{155}$ H. Abreu, ${ }^{154}$ R. Abreu, ${ }^{118}$ Y. Abulaiti, ${ }^{148 a, 148 b}$ B. S. Acharya, ${ }^{167 a, 167 b, b}$ S. Adachi, ${ }^{157}$ L. Adamczyk, ${ }^{41 a}$ J. Adelman, ${ }^{110}$ M. Adersberger, ${ }^{102}$ T. Adye, ${ }^{133}$ A. A. Affolder, ${ }^{139}$ T. Agatonovic-Jovin, ${ }^{14}$ C. Agheorghiesei, ${ }^{28 c}$

J. A. Aguilar-Saavedra, ${ }^{128 a, 128 f}$ S. P. Ahlen, ${ }^{24}$ F. Ahmadov, ${ }^{68, c}$ G. Aielli, ${ }^{135 a, 135 b}$ S. Akatsuka, ${ }^{71}$ H. Akerstedt, ${ }^{148 a, 148 b}$ T. P. A. Åkesson, ${ }^{84}$ E. Akilli, ${ }^{52}$ A. V. Akimov, ${ }^{98}$ G. L. Alberghi, ${ }^{22 a, 22 b}$ J. Albert, ${ }^{172}$ P. Albicocco,${ }^{50}$ M. J. Alconada Verzini, ${ }^{74}$ S. C. Alderweireldt, ${ }^{108}$ M. Aleksa, ${ }^{32}$ I. N. Aleksandrov, ${ }^{68}$ C. Alexa, ${ }^{28 b}$ G. Alexander, ${ }^{155}$ T. Alexopoulos,${ }^{10}$ M. Alhroob, ${ }^{115}$ B. Ali ${ }^{130}$ M. Aliev ${ }^{76 a, 76 b}$ G. Alimonti, ${ }^{94 a}$ J. Alison, ${ }^{33}$ S. P. Alkire, ${ }^{38}$ B. M. M. Allbrooke, ${ }^{151}$ B. W. Allen, ${ }^{118}$ P. P. Allport, ${ }^{19}$ A. Aloisio, ${ }^{106 a, 106 \mathrm{~b}}$ A. Alonso, ${ }^{39}$ F. Alonso, ${ }^{74}$ C. Alpigiani, ${ }^{140}$ A. A. Alshehri, ${ }^{56}$ M. Alstaty, ${ }^{88}$ B. Alvarez Gonzalez, ${ }^{32}$ D. Álvarez Piqueras, ${ }^{170}$ M. G. Alviggi, ${ }^{106 a, 106 b}$ B. T. Amadio, ${ }^{16}$ Y. Amaral Coutinho, ${ }^{26 a}$ C. Amelung, ${ }^{25}$ D. Amidei ${ }^{92}$ S. P. Amor Dos Santos, ${ }^{128 a, 128 c}$ A. Amorim, ${ }^{128 a, 128 b}$ S. Amoroso, ${ }^{32}$ G. Amundsen, ${ }^{25}$ C. Anastopoulos, ${ }^{141}$ L. S. Ancu, ${ }^{52}$

N. Andari, ${ }^{19}$ T. Andeen, ${ }^{11}$ C. F. Anders, ${ }^{60 b}$ J. K. Anders, ${ }^{77}$ K. J. Anderson, ${ }^{33}$ A. Andreazza, ${ }^{94 a, 94 b}$ V. Andrei, ${ }^{60 a}$ S. Angelidakis, ${ }^{9}$ I. Angelozzi, ${ }^{109}$ A. Angerami, ${ }^{38}$ A. V. Anisenkov, ${ }^{11, d}$ N. Anjos, ${ }^{13}$ A. Annovi, ${ }^{126 a, 126 b}$ C. Antel, ${ }^{60 a}$ M. Antonelli, ${ }^{50}$ A. Antonov, ${ }^{100, a}$ D. J. Antrim, ${ }^{166}$ F. Anulli, ${ }^{134 a}$ M. Aoki,${ }^{69}$ L. Aperio Bella, ${ }^{32}$ G. Arabidze,${ }^{93}$ Y. Arai, ${ }^{69}$ J. P. Araque, ${ }^{128 a}$ V. Araujo Ferraz, ${ }^{26 a}$ A. T. H. Arce, ${ }^{48}$ R. E. Ardell,${ }^{80}$ F. A. Arduh, ${ }^{74}$ J-F. Arguin, ${ }^{97}$ S. Argyropoulos, ${ }^{66}$ M. Arik, ${ }^{20 a}$ A. J. Armbruster, ${ }^{32}$ L. J. Armitage, ${ }^{79}$ O. Arnaez, ${ }^{161}$ H. Arnold, ${ }^{51}$ M. Arratia, ${ }^{30}$ O. Arslan ${ }^{23}$ A. Artamonov, ${ }^{99}$ G. Artoni, ${ }^{122}$ S. Artz ${ }^{86}$ S. Asai ${ }^{157}$ N. Asbah,${ }^{45}$ A. Ashkenazi,${ }^{155}$ L. Asquith ${ }^{151}$ K. Assamagan, ${ }^{27}$ R. Astalos, ${ }^{146 a}$ M. Atkinson, ${ }^{169}$ N. B. Atlay, ${ }^{143}$ K. Augsten, ${ }^{130}$ G. Avolio, ${ }^{32}$ B. Axen, ${ }^{16}$ M. K. Ayoub, ${ }^{119}$ G. Azuelos, ${ }^{97, e}$ A. E. Baas, ${ }^{60 a}$ M. J. Baca, ${ }^{19}$ H. Bachacou, ${ }^{138}$ K. Bachas, ${ }^{76 a, 76 b}$ M. Backes, ${ }^{122}$ M. Backhaus, ${ }^{32}$ P. Bagnaia, ${ }^{134 a, 134 b}$ M. Bahmani, ${ }^{42}$ H. Bahrasemani, ${ }^{144}$ J. T. Baines, ${ }^{133}$ M. Bajic, ${ }^{39}$ O. K. Baker, ${ }^{179}$ E. M. Baldin, ${ }^{11, d}$ P. Balek, ${ }^{175}$ F. Balli, ${ }^{138}$ W. K. Balunas, ${ }^{124}$ E. Banas, ${ }^{42}$ A. Bandyopadhyay, ${ }^{23}$ Sw. Banerjee, ${ }^{176, f}$ A. A. E. Bannoura, ${ }^{178}$ L. Barak,${ }^{32}$ E. L. Barberio, ${ }^{91}$ D. Barberis, ${ }^{53 a, 53 b}$ M. Barbero, ${ }^{88}$ T. Barillari, ${ }^{103}$ M-S Barisits, ${ }^{32}$ J. T. Barkeloo, ${ }^{118}$ T. Barklow, ${ }^{145}$ N. Barlow, ${ }^{30}$ S. L. Barnes, ${ }^{36 c}$ B. M. Barnett, ${ }^{133}$ R. M. Barnett, ${ }^{16}$ Z. Barnovska-Blenessy, ${ }^{36 a}$ A. Baroncelli, ${ }^{136 a}$ G. Barone ${ }^{25}$ A. J. Barr, ${ }^{122}$ L. Barranco Navarro, ${ }^{170}$ F. Barreiro, ${ }^{85}$ J. Barreiro Guimarães da Costa, ${ }^{35 a}$ R. Bartoldus, ${ }^{145}$ A. E. Barton, ${ }^{75}$ P. Bartos, ${ }^{146 a}$ A. Basalaev, ${ }^{125}$

A. Bassalat, ${ }^{19, g}$ R. L. Bates, ${ }^{56}$ S. J. Batista, ${ }^{161}$ J. R. Batley, ${ }^{30}$ M. Battaglia, ${ }^{139}$ M. Bauce, ${ }^{134 a, 134 b}$ F. Bauer, ${ }^{138}$ H. S. Bawa, ${ }^{145, h}$ J. B. Beacham, ${ }^{113}$ M. D. Beattie, ${ }^{75}$ T. Beau, ${ }^{83}$ P. H. Beauchemin, ${ }^{165}$ P. Bechtle,${ }^{23}$ H. P. Beck, ${ }^{18, \mathrm{i}}$ H. C. Beck,${ }^{57}$ K. Becker, ${ }^{122}$ M. Becker ${ }^{86}$ M. Beckingham, ${ }^{173}$ C. Becot ${ }^{112}$ A. J. Beddall, ${ }^{20 d}$ A. Beddall, ${ }^{20 b}$ V. A. Bednyakov,${ }^{68}$ M. Bedognetti, ${ }^{109}$ C. P. Bee,${ }^{150}$ T. A. Beermann, ${ }^{32}$ M. Begalli, ${ }^{26 a}$ M. Begel,${ }^{27}$ J. K. Behr, ${ }^{45}$ A. S. Bell, ${ }^{81}$ G. Bella, ${ }^{155}$ L. Bellagamba, ${ }^{22 a}$ A. Bellerive,${ }^{31}$ M. Bellomo, ${ }^{154}$ K. Belotskiy, ${ }^{100}$ O. Beltramello, ${ }^{32}$ N. L. Belyaev, ${ }^{100}$ O. Benary, ${ }^{155, a}$ D. Benchekroun, ${ }^{137 a}$ M. Bender, ${ }^{102}$ K. Bendtz, ${ }^{148 a, 148 b}$ N. Benekos, ${ }^{10}$ Y. Benhammou, ${ }^{155}$ E. Benhar Noccioli, ${ }^{179}$ J. Benitez, ${ }^{66}$ D. P. Benjamin,${ }^{48}$ M. Benoit, ${ }^{52}$ J. R. Bensinger, ${ }^{25}$ S. Bentvelsen, ${ }^{109}$ L. Beresford, ${ }^{122}$ M. Beretta, ${ }^{50}$ D. Berge, ${ }^{109}$ E. Bergeaas Kuutmann, ${ }^{168}$ N. Berger, ${ }^{5}$ J. Beringer, ${ }^{16}$ S. Berlendis, ${ }^{58}$ N. R. Bernard,${ }^{89}$ G. Bernardi, ${ }^{83}$ C. Bernius, ${ }^{145}$ F. U. Bernlochner, ${ }^{23}$ T. Berry,${ }^{80}$ P. Berta, ${ }^{131}$ C. Bertella, ${ }^{35 a}$ G. Bertoli, ${ }^{148 a, 148 b}$ F. Bertolucci, ${ }^{126 a, 126 b}$ I. A. Bertram, ${ }^{75}$ C. Bertsche, ${ }^{45}$ D. Bertsche,${ }^{15}$ G. J. Besjes, ${ }^{39}$ O. Bessidskaia Bylund, ${ }^{148 a, 148 b}$ M. Bessner, ${ }^{45}$ N. Besson, ${ }^{138}$ C. Betancourt, ${ }^{51}$ A. Bethani, ${ }^{87}$ S. Bethke, ${ }^{103}$ 
A. J. Bevan, ${ }^{79}$ J. Beyer,${ }^{103}$ R. M. Bianchi, ${ }^{127}$ O. Biebel,${ }^{102}$ D. Biedermann, ${ }^{17}$ R. Bielski, ${ }^{87}$ K. Bierwagen,${ }^{86}$ N. V. Biesuz, ${ }^{126 a, 126 b}$ M. Biglietti, ${ }^{136 a}$ T. R. V. Billoud,${ }^{97}$ H. Bilokon, ${ }^{50}$ M. Bindi, ${ }^{57}$ A. Bingul, ${ }^{20 b}$ C. Bini, ${ }^{134 a, 134 b}$ S. Biondi, ${ }^{22 a, 22 b}$ T. Bisanz, ${ }^{57}$ C. Bittrich, ${ }^{47}$ D. M. Bjergaard, ${ }^{48}$ C. W. Black, ${ }^{152}$ J. E. Black, ${ }^{145}$ K. M. Black, ${ }^{24}$ R. E. Blair, ${ }^{6}$ T. Blazek, ${ }^{146 a}$ I. Bloch, ${ }^{45}$ C. Blocker, ${ }^{25}$ A. Blue,${ }^{56}$ W. Blum, ${ }^{86, a}$ U. Blumenschein, ${ }^{79}$ S. Blunier, ${ }^{34 a}$ G. J. Bobbink, ${ }^{109}$ V. S. Bobrovnikov, ${ }^{11, d}$ S. S. Bocchetta, ${ }^{84}$ A. Bocci,${ }^{48}$ C. Bock, ${ }^{102}$ M. Boehler, ${ }^{51}$ D. Boerner, ${ }^{178}$ D. Bogavac, ${ }^{102}$ A. G. Bogdanchikov, ${ }^{111}$ C. Bohm, ${ }^{148 a}$ V. Boisvert,${ }^{80}$ P. Bokan, ${ }^{168, j}$ T. Bold, ${ }^{41 a}$ A. S. Boldyrev, ${ }^{101}$ A. E. Bolz,${ }^{60 b}$ M. Bomben,${ }^{83}$ M. Bona, ${ }^{79}$ M. Boonekamp, ${ }^{138}$ A. Borisov, ${ }^{132}$ G. Borissov, ${ }^{75}$ J. Bortfeldt,${ }^{32}$ D. Bortoletto, ${ }^{122}$ V. Bortolotto, ${ }^{62 a, 62 b, 62 c}$ D. Boscherini, ${ }^{22 a}$ M. Bosman, ${ }^{13}$ J. D. Bossio Sola, ${ }^{29}$ J. Boudreau, ${ }^{127} \mathrm{~J}$. Bouffard, ${ }^{2}$ E. V. Bouhova-Thacker, ${ }^{75}$ D. Boumediene, ${ }^{37}$ C. Bourdarios, ${ }^{119}$ S. K. Boutle,${ }^{56}$ A. Boveia, ${ }^{113}$ J. Boyd ${ }^{32}$ I. R. Boyko, ${ }^{68}$ J. Bracinik, ${ }^{19}$ A. Brandt, ${ }^{8}$ G. Brandt, ${ }^{57}$ O. Brandt, ${ }^{60 a}$ U. Bratzler, ${ }^{158}$ B. Brau, ${ }^{89}$ J. E. Brau, ${ }^{118}$ W. D. Breaden Madden, ${ }^{56}$ K. Brendlinger, ${ }^{45}$ A. J. Brennan, ${ }^{91}$ L. Brenner, ${ }^{109}$ R. Brenner, ${ }^{168}$ S. Bressler, ${ }^{175}$ D. L. Briglin, ${ }^{19}$ T. M. Bristow, ${ }^{49}$ D. Britton,${ }^{56}$ D. Britzger, ${ }^{45}$ F. M. Brochu ${ }^{30}$ I. Brock, ${ }^{23}$ R. Brock,${ }^{93}$ G. Brooijmans, ${ }^{38}$ T. Brooks, ${ }^{80}$ W. K. Brooks ${ }^{34 b}$ J. Brosamer, ${ }^{16}$ E. Brost, ${ }^{110}$ J. H Broughton, ${ }^{19}$ P. A. Bruckman de Renstrom, ${ }^{42}$ D. Bruncko, ${ }^{146 b}$ A. Bruni, ${ }^{22 a}$ G. Bruni, ${ }^{22 a}$ L. S. Bruni, ${ }^{109}$ BH Brunt, ${ }^{30}$ M. Bruschi, ${ }^{22 a}$ N. Bruscino, ${ }^{23}$ P. Bryant, ${ }^{33}$ L. Bryngemark, ${ }^{45}$ T. Buanes,${ }^{15}$ Q. Buat, ${ }^{144}$ P. Buchholz, ${ }^{143}$ A. G. Buckley, ${ }^{56}$ I. A. Budagov, ${ }^{68}$ F. Buehrer, ${ }^{51}$ M. K. Bugge, ${ }^{121}$ O. Bulekov, ${ }^{100}$ D. Bullock, ${ }^{8}$ T. J. Burch, ${ }^{110}$ S. Burdin, ${ }^{77}$ C. D. Burgard, ${ }^{51}$ A. M. Burger, ${ }^{5}$ B. Burghgrave, ${ }^{110}$ K. Burka, ${ }^{42}$ S. Burke, ${ }^{133}$ I. Burmeister, ${ }^{46}$ J. T. P. Burr, ${ }^{122}$ E. Busato, ${ }^{37}$ D. Büscher, ${ }^{51}$ V. Büscher ${ }^{86}$ P. Bussey, ${ }^{56}$ J. M. Butler, ${ }^{24}$ C. M. Buttar, ${ }^{56}$ J. M. Butterworth ${ }^{81}$ P. Butti, ${ }^{32}$ W. Buttinger, ${ }^{27}$

A. Buzatu, ${ }^{35 c}$ A. R. Buzykaev, ${ }^{111, d}$ S. Cabrera Urbán, ${ }^{170}$ D. Caforio, ${ }^{130}$ V. M. Cairo, ${ }^{40 a, 40 b}$ O. Cakir, ${ }^{4 a}$ N. Calace, ${ }^{52}$ P. Calafiura, ${ }^{16}$ A. Calandri, ${ }^{88}$ G. Calderini, ${ }^{83}$ P. Calfayan, ${ }^{64}$ G. Callea, ${ }^{40 a}{ }^{40 b}$ L. P. Caloba, ${ }^{26 a}$ S. Calvente Lopez, ${ }^{85}$ D. Calvet,${ }^{37}$ S. Calvet, ${ }^{37}$ T. P. Calvet, ${ }^{88}$ R. Camacho Toro, ${ }^{33}$ S. Camarda,${ }^{32}$ P. Camarri, ${ }^{135 a, 135 b}$ D. Cameron, ${ }^{121}$ R. Caminal Armadans, ${ }^{169}$ C. Camincher, ${ }^{58}$ S. Campana, ${ }^{32}$ M. Campanelli, ${ }^{81}$ A. Camplani, ${ }^{94 a, 94 b}$ A. Campoverde,${ }^{143}$ V. Canale, ${ }^{106 a, 106 \mathrm{~b}}$ M. Cano Bret, ${ }^{36 \mathrm{c}}$ J. Cantero, ${ }^{116}$ T. Cao, ${ }^{155}$ M. D. M. Capeans Garrido, ${ }^{32}$ I. Caprini, ${ }^{28 b}$ M. Caprini, ${ }^{28 b}$ M. Capua ${ }^{40 a, 40 b}$ R. M. Carbone, ${ }^{38}$ R. Cardarelli, ${ }^{135 a}$ F. Cardillo, ${ }^{51}$ I. Carli, ${ }^{131}$ T. Carli, ${ }^{32}$ G. Carlino, ${ }^{106 a}$ B. T. Carlson, ${ }^{127}$ L. Carminati, ${ }^{94 a, 94 b}$

R. M. D. Carney, ${ }^{148 a, 148 b}$ S. Caron, ${ }^{108}$ E. Carquin, ${ }^{34 b}$ S. Carrá, ${ }^{94 a, 94 b}$ G. D. Carrillo-Montoya, ${ }^{32}$ J. Carvalho, ${ }^{128 a, 128 c}$ D. Casadei, ${ }^{19}$ M. P. Casado, ${ }^{13, k}$ M. Casolino, ${ }^{13}$ D. W. Casper, ${ }^{166}$ R. Castelijn, ${ }^{109}$ V. Castillo Gimenez, ${ }^{170}$ N. F. Castro, ${ }^{128 a, 1}$ A. Catinaccio, ${ }^{32}$ J. R. Catmore, ${ }^{121}$ A. Cattai, ${ }^{32}$ J. Caudron, ${ }^{23}$ V. Cavaliere, ${ }^{169}$ E. Cavallaro, ${ }^{13}$ D. Cavalli, ${ }^{94 a}$

M. Cavalli-Sforza, ${ }^{13}$ V. Cavasinni, ${ }^{126 a, 126 b}$ E. Celebi, ${ }^{20 \mathrm{a}}$ F. Ceradini, ${ }^{136 a, 136 \mathrm{~b}}$ L. Cerda Alberich, ${ }^{170}$ A. S. Cerqueira, ${ }^{26 \mathrm{~b}}$ A. Cerri, ${ }^{151}$ L. Cerrito, ${ }^{135 a, 135 b}$ F. Cerutti, ${ }^{16}$ A. Cervelli, ${ }^{18}$ S. A. Cetin, ${ }^{20 c}$ A. Chafaq, ${ }^{137 a}$ D. Chakraborty, ${ }^{110}$ S. K. Chan,${ }^{59}$ W. S. Chan, ${ }^{109}$ Y. L. Chan, ${ }^{62 a}$ P. Chang, ${ }^{169}$ J. D. Chapman, ${ }^{30}$ D. G. Charlton, ${ }^{19}$ C. C. Chau, ${ }^{161}$ C. A. Chavez Barajas, ${ }^{151}$

S. Che, ${ }^{113}$ S. Cheatham, ${ }^{167 a, 167 \mathrm{c}}$ A. Chegwidden, ${ }^{93}$ S. Chekanov, ${ }^{6}$ S. V. Chekulaev, ${ }^{163 a}$ G. A. Chelkov, ${ }^{68, m}$

M. A. Chelstowska, ${ }^{32}$ C. Chen, ${ }^{67}$ H. Chen, ${ }^{27}$ J. Chen, ${ }^{36 a}$ S. Chen, ${ }^{35 b}$ S. Chen ${ }^{157}$ X. Chen,${ }^{35, n}$ Y. Chen, ${ }^{70}$ H. C. Cheng, ${ }^{92}$ H. J. Cheng, ${ }^{35 a}$ A. Cheplakov, ${ }^{68}$ E. Cheremushkina, ${ }^{132}$ R. Cherkaoui El Moursli, ${ }^{137 e}$ E. Cheu, ${ }^{7}$ K. Cheung, ${ }^{63}$ L. Chevalier, ${ }^{138}$ V. Chiarella, ${ }^{50}$ G. Chiarelli, ${ }^{126 a, 126 b}$ G. Chiodini, ${ }^{76 a}$ A. S. Chisholm, ${ }^{32}$ A. Chitan, ${ }^{28 b}$ Y. H. Chiu, ${ }^{172}$ M. V. Chizhov, ${ }^{68}$ K. Choi ${ }^{64}$ A. R. Chomont,${ }^{37}$ S. Chouridou, ${ }^{156}$ V. Christodoulou, ${ }^{81}$ D. Chromek-Burckhart, ${ }^{32}$ M. C. Chu, ${ }^{62 a}$ J. Chudoba, ${ }^{129}$ A. J. Chuinard, ${ }^{90}$ J. J. Chwastowski, ${ }^{42}$ L. Chytka, ${ }^{117}$ A. K. Ciftci, ${ }^{4 a}$ D. Cinca,${ }^{46}$ V. Cindro, ${ }^{78}$ I. A. Cioara, ${ }^{23}$ C. Ciocca, ${ }^{22 a, 22 b}$ A. Ciocio, ${ }^{16}$ F. Cirotto, ${ }^{106 a, 106 b}$ Z. H. Citron, ${ }^{175}$ M. Citterio, ${ }^{94 a}$ M. Ciubancan, ${ }^{28 b}$ A. Clark, ${ }^{52}$ B. L. Clark,${ }^{59}$ M. R. Clark, ${ }^{38}$ P. J. Clark,${ }^{49}$ R. N. Clarke,${ }^{16}$ C. Clement, ${ }^{148 a, 148 b}$ Y. Coadou, ${ }^{88}$ M. Cobal,${ }^{167 a, 167 c}$ A. Coccaro, ${ }^{52}$ J. Cochran, ${ }^{67}$ L. Colasurdo, ${ }^{108}$ B. Cole, ${ }^{38}$ A. P. Colijn, ${ }^{109}$ J. Collot, ${ }^{58}$ T. Colombo, ${ }^{166}$ P. Conde Muiño, ${ }^{128 a, 128 b}$ E. Coniavitis, ${ }^{51}$ S. H. Connell, ${ }^{147 b}$ I. A. Connelly, ${ }^{87}$ S. Constantinescu, ${ }^{28 b}$ G. Conti, ${ }^{32}$ F. Conventi, ${ }^{106 a, 0}$ M. Cooke, ${ }^{16}$ A. M. Cooper-Sarkar, ${ }^{122}$ F. Cormier, ${ }^{171}$ K. J. R. Cormier, ${ }^{161}$ M. Corradi, ${ }^{134 a, 134 b}$ F. Corriveau, ${ }^{90, p}$ A. Cortes-Gonzalez, ${ }^{32}$ G. Cortiana, ${ }^{103}$ G. Costa, ${ }^{94 a}$ M. J. Costa, ${ }^{170}$ D. Costanzo, ${ }^{141}$ G. Cottin, ${ }^{30}$ G. Cowan, ${ }^{80}$ B. E. Cox,${ }^{87}$ K. Cranmer, ${ }^{12}$ S. J. Crawley, ${ }^{56}$ R. A. Creager, ${ }^{124}$ G. Cree, ${ }^{31}$ S. Crépé-Renaudin, ${ }^{58}$ F. Crescioli, ${ }^{83}$ W. A. Cribbs, ${ }^{148 a, 148 b}$ M. Cristinziani, ${ }^{23}$ V. Croft, ${ }^{108}$ G. Crosetti, ${ }^{40 a, 40 b}$ A. Cueto, ${ }^{85}$

T. Cuhadar Donszelmann, ${ }^{141}$ A. R. Cukierman, ${ }^{145}$ J. Cummings, ${ }^{179}$ M. Curatolo, ${ }^{50}$ J. Cúth ${ }^{86}$ S. Czekierda, ${ }^{42}$

P. Czodrowski, ${ }^{32}$ G. D'amen, ${ }^{22 a, 22 b}$ S. D'Auria ${ }^{56}$ L. D'eramo, ${ }^{83}$ M. D’Onofrio, ${ }^{77}$ M. J. Da Cunha Sargedas De Sousa, ${ }^{128 a, 128 b}$ C. Da Via, ${ }^{87}$ W. Dabrowski, ${ }^{41 a}$ T. Dado, ${ }^{146 a}$ T. Dai,${ }^{92}$ O. Dale, ${ }^{15}$ F. Dallaire, ${ }^{97}$ C. Dallapiccola, ${ }^{89}$ M. Dam,${ }^{39}$ J. R. Dandoy, ${ }^{124}$ M. F. Daneri, ${ }^{29}$ N. P. Dang, ${ }^{176}$ A. C. Daniells, ${ }^{19}$ N. S. Dann, ${ }^{87}$ M. Danninger, ${ }^{171}$ M. Dano Hoffmann, ${ }^{138}$ V. Dao, ${ }^{150}$ G. Darbo, ${ }^{53 a}$ S. Darmora, ${ }^{8}$ J. Dassoulas, ${ }^{3}$ A. Dattagupta, ${ }^{118}$ T. Daubney, ${ }^{45}$ W. Davey, ${ }^{23}$ C. David ${ }^{45}$ T. Davidek,${ }^{131}$

D. R. Davis, ${ }^{48}$ P. Davison, ${ }^{81}$ E. Dawe, ${ }^{91}$ I. Dawson, ${ }^{141}$ K. De,${ }^{8}$ R. de Asmundis, ${ }^{106 a}$ A. De Benedetti, ${ }^{115}$ S. De Castro, ${ }^{22 a, 22 b}$ S. De Cecco, ${ }^{83}$ N. De Groot, ${ }^{108}$ P. de Jong, ${ }^{109}$ H. De la Torre, ${ }^{93}$ F. De Lorenzi, ${ }^{67}$ A. De Maria, ${ }^{57}$ D. De Pedis, ${ }^{134 a}$ 
A. De Salvo, ${ }^{134 a}$ U. De Sanctis, ${ }^{135 a, 135 b}$ A. De Santo, ${ }^{151}$ K. De Vasconcelos Corga ${ }^{88}$ J. B. De Vivie De Regie, ${ }^{119}$ W. J. Dearnaley, ${ }^{75}$ R. Debbe, ${ }^{27}$ C. Debenedetti, ${ }^{139}$ D. V. Dedovich, ${ }^{68}$ N. Dehghanian, ${ }^{3}$ I. Deigaard, ${ }^{109}$ M. Del Gaudio,,${ }^{40,40 b}$

J. Del Peso, ${ }^{85}$ D. Delgove, ${ }^{119}$ F. Deliot,${ }^{138}$ C. M. Delitzsch, ${ }^{7}$ A. Dell'Acqua,${ }^{32}$ L. Dell'Asta, ${ }^{24}$ M. Dell'Orso, ${ }^{126 a, 126 b}$ M. Della Pietra, ${ }^{106 a, 106 b}$ D. della Volpe,${ }^{52}$ M. Delmastro, ${ }^{5}$ C. Delporte, ${ }^{119}$ P. A. Delsart, ${ }^{58}$ D. A. DeMarco, ${ }^{161}$ S. Demers, ${ }^{179}$ M. Demichev ${ }^{68}$ A. Demilly, ${ }^{83}$ S. P. Denisov, ${ }^{132}$ D. Denysiuk, ${ }^{138}$ D. Derendarz,${ }^{42}$ J. E. Derkaoui, ${ }^{137 d}$ F. Derue,${ }^{83}$ P. Dervan, ${ }^{77}$ K. Desch, ${ }^{23}$ C. Deterre, ${ }^{45}$ K. Dette, ${ }^{46}$ M. R. Devesa, ${ }^{29}$ P. O. Deviveiros, ${ }^{32}$ A. Dewhurst, ${ }^{133}$ S. Dhaliwal, ${ }^{25}$ F. A. Di Bello, ${ }^{52}$ A. Di Ciaccio, ${ }^{135 a, 135 b}$ L. Di Ciaccio, ${ }^{5}$ W. K. Di Clemente, ${ }^{124}$ C. Di Donato, ${ }^{106 a, 106 b}$ A. Di Girolamo,${ }^{32}$ B. Di Girolamo, ${ }^{32}$ B. Di Micco, ${ }^{136 a, 136 b}$ R. Di Nardo,${ }^{32}$ K. F. Di Petrillo, ${ }^{59}$ A. Di Simone, ${ }^{51}$ R. Di Sipio, ${ }^{161}$ D. Di Valentino, ${ }^{31}$ C. Diaconu ${ }^{88}$ M. Diamond ${ }^{161}$ F. A. Dias, ${ }^{39}$ M. A. Diaz,${ }^{34 a}$ E. B. Diehl,${ }^{92}$ J. Dietrich, ${ }^{17}$ S. Díez Cornell, ${ }^{45}$ A. Dimitrievska, ${ }^{14}$

J. Dingfelder, ${ }^{23}$ P. Dita, ${ }^{28 \mathrm{~b}}$ S. Dita, ${ }^{28 \mathrm{~b}}$ F. Dittus, ${ }^{32}$ F. Djama ${ }^{88}$ T. Djobava,${ }^{54 \mathrm{~b}}$ J. I. Djuvsland, ${ }^{60 \mathrm{a}}$ M. A. B. do Vale, ${ }^{26 \mathrm{c}}$ D. Dobos, ${ }^{32}$ M. Dobre, ${ }^{28 b}$ C. Doglioni, ${ }^{84}$ J. Dolejsi, ${ }^{131}$ Z. Dolezal, ${ }^{131}$ M. Donadelli, ${ }^{26 \mathrm{~d}}$ S. Donati, ${ }^{126 a, 126 b}$ P. Dondero, ${ }^{123 a, 123 b}$ J. Donini, ${ }^{37}$ J. Dopke, ${ }^{133}$ A. Doria, ${ }^{106 a}$ M. T. Dova,${ }^{74}$ A. T. Doyle,${ }^{56}$ E. Drechsler, ${ }^{57}$ M. Dris, ${ }^{10}$ Y. Du, ${ }^{36 b}$ J. Duarte-Campderros, ${ }^{155}$ A. Dubreuil,${ }^{52}$ E. Duchovni, ${ }^{175}$ G. Duckeck, ${ }^{102}$ A. Ducourthial, ${ }^{83}$ O. A. Ducu, ${ }^{97, q}$ D. Duda, ${ }^{109}$ A. Dudarev, ${ }^{32}$ A. Chr. Dudder, ${ }^{86}$ E. M. Duffield, ${ }^{16}$ L. Duflot, ${ }^{119}$ M. Dührssen, ${ }^{32}$ M. Dumancic, ${ }^{175}$ A. E. Dumitriu, ${ }^{28 b}$ A. K. Duncan, ${ }^{56}$ M. Dunford, ${ }^{60 \mathrm{a}}$ H. Duran Yildiz, ${ }^{4 \mathrm{a}}$ M. Düren, ${ }^{55}$ A. Durglishvili, ${ }^{54 \mathrm{~b}}$ D. Duschinger, ${ }^{47}$ B. Dutta, ${ }^{45}$ M. Dyndal, ${ }^{45}$ B. S. Dziedzic, ${ }^{42}$ C. Eckardt,${ }^{45}$ K. M. Ecker ${ }^{103}$ R. C. Edgar,${ }^{92}$ T. Eifert, ${ }^{32}$ G. Eigen, ${ }^{15}$ K. Einsweiler, ${ }^{16}$ T. Ekelof, ${ }^{168}$ M. El Kacimi, ${ }^{137 \mathrm{c}}$ R. El Kosseifi, ${ }^{88}$ V. Ellajosyula ${ }^{88}$ M. Ellert, ${ }^{168}$ S. Elles, ${ }^{5}$ F. Ellinghaus, ${ }_{178}^{178}$ A. A. Elliot, ${ }^{172}$ N. Ellis, ${ }^{32}$ J. Elmsheuser, ${ }^{27}$ M. Elsing, ${ }^{32}$ D. Emeliyanov, ${ }^{133}$ Y. Enari, ${ }^{157}$ O. C. Endner, ${ }^{86}$ J. S. Ennis, ${ }^{173}$ J. Erdmann, ${ }^{46}$ A. Ereditato, ${ }^{18}$ M. Ernst, ${ }^{27}$ S. Errede,${ }^{169}$ M. Escalier, ${ }^{119}$ C. Escobar,${ }^{170}$ B. Esposito, ${ }^{50}$ O. Estrada Pastor ${ }^{170}$ A. I. Etienvre, ${ }^{138}$ E. Etzion, ${ }^{155}$ H. Evans,${ }^{64}$ A. Ezhilov, ${ }^{125}$ M. Ezzi ${ }^{137 \mathrm{e}}$ F. Fabbri, ${ }^{22 a, 22 \mathrm{~b}}$ L. Fabbri, ${ }^{22 a, 22 b}$ V. Fabiani, ${ }^{108}$ G. Facini, ${ }^{81}$ R. M. Fakhrutdinov, ${ }^{132}$ S. Falciano, ${ }^{134 a}$ R. J. Falla, ${ }^{81}$ J. Faltova, ${ }^{32}$ Y. Fang, ${ }^{35 a}$ M. Fanti, ${ }^{94 a}, 94 b$ A. Farbin, ${ }^{8}$ A. Farilla, ${ }^{136 a}$ C. Farina, ${ }^{127}$ E. M. Farina, ${ }^{123 a, 123 b}$ T. Farooque, ${ }^{93}$ S. Farrell,${ }^{16}$ S. M. Farrington, ${ }^{173}$ P. Farthouat,${ }^{32}$ F. Fassi,${ }^{137 \mathrm{e}}$ P. Fassnacht,${ }^{32}$ D. Fassouliotis, ${ }^{9}$ M. Faucci Giannelli, ${ }^{80}$ A. Favareto, ${ }^{53 a, 53 \mathrm{~b}}$ W. J. Fawcett, ${ }^{122}$ L. Fayard, ${ }^{119}$ O. L. Fedin, ${ }^{125, \mathrm{r}}$ W. Fedorko, ${ }^{171}$ S. Feigl, ${ }^{121}$ L. Feligioni, ${ }^{88}$ C. Feng, ${ }^{36 b}$ E. J. Feng, ${ }^{32}$ H. Feng, ${ }^{92}$ M. J. Fenton, ${ }^{56}$ A. B. Fenyuk, ${ }^{132}$ L. Feremenga, ${ }^{8}$

P. Fernandez Martinez, ${ }^{170}$ S. Fernandez Perez, ${ }^{13}$ J. Ferrando, ${ }^{45}$ A. Ferrari, ${ }^{168}$ P. Ferrari, ${ }^{109}$ R. Ferrari, ${ }^{123 a}$

D. E. Ferreira de Lima, ${ }^{60 \mathrm{~b}}$ A. Ferrer ${ }^{170}$ D. Ferrere ${ }^{52}$ C. Ferretti,${ }^{92}$ F. Fiedler, ${ }^{86}$ A. Filipčičc ${ }^{78}$ M. Filipuzzi ${ }^{45}$ F. Filthaut, ${ }^{108}$ M. Fincke-Keeler, ${ }^{172}$ K. D. Finelli, ${ }^{152}$ M. C. N. Fiolhais, ${ }^{128 a, 128 c, s}$ L. Fiorini, ${ }^{170}$ A. Fischer, ${ }^{2}$ C. Fischer, ${ }^{13}$ J. Fischer, ${ }^{178}$ W. C. Fisher ${ }^{93}$ N. Flaschel,${ }^{45}$ I. Fleck,${ }^{143}$ P. Fleischmann, ${ }^{92}$ R. R. M. Fletcher, ${ }^{124}$ T. Flick, ${ }^{178}$ B. M. Flierl, ${ }^{102}$ L. R. Flores Castillo, ${ }^{62 a}$ M. J. Flowerdew, ${ }^{103}$ G. T. Forcolin, ${ }^{87}$ A. Formica, ${ }^{138}$ F. A. Förster, ${ }^{13}$ A. Forti, ${ }^{87}$ A. G. Foster, ${ }^{19}$ D. Fournier, ${ }^{119}$ H. Fox, ${ }^{75}$ S. Fracchia, ${ }^{141}$ P. Francavilla, ${ }^{83}$ M. Franchini, ${ }^{22 a, 22 b}$ S. Franchino, ${ }^{60 a}$ D. Francis,${ }^{32}$ L. Franconi, ${ }^{121}$ M. Franklin, ${ }^{59}$ M. Frate, ${ }^{166}$ M. Fraternali, ${ }^{123 a, 123 b}$ D. Freeborn, ${ }^{81}$ S. M. Fressard-Batraneanu, ${ }^{32}$ B. Freund, ${ }^{97}$ D. Froidevaux, ${ }^{32}$ J. A. Frost, ${ }^{122}$ C. Fukunaga, ${ }^{158}$ T. Fusayasu, ${ }^{104}$ J. Fuster, ${ }^{170}$ C. Gabaldon, ${ }^{58}$ O. Gabizon, ${ }^{154}$ A. Gabrielli, ${ }^{22 a, 22 b}$ A. Gabrielli, ${ }^{16}$ G. P. Gach, ${ }^{41 \mathrm{a}}$ S. Gadatsch, ${ }^{32}$ S. Gadomski, ${ }^{80}$ G. Gagliardi, ${ }^{53 \mathrm{a}, 53 \mathrm{~b}}$ L. G. Gagnon, ${ }^{97}$ C. Galea, ${ }^{108}$ B. Galhardo, ${ }^{128 \mathrm{a}, 128 \mathrm{c}}$ E. J. Gallas, ${ }^{122}$ B. J. Gallop, ${ }^{133}$ P. Gallus, ${ }^{130}$ G. Galster, ${ }^{39}$ K. K. Gan, ${ }^{113}$ S. Ganguly, ${ }^{37}$ Y. Gao, ${ }^{77}$ Y. S. Gao, ${ }^{145, h}$ F. M. Garay Walls, ${ }^{49}$ C. García ${ }^{170}$ J. E. García Navarro, ${ }^{170}$ J. A. García Pascual,${ }^{35 a}$ M. Garcia-Sciveres, ${ }^{16}$ R. W. Gardner, ${ }^{33}$ N. Garelli, ${ }^{145}$ V. Garonne, ${ }^{121}$ A. Gascon Bravo,${ }^{45}$ K. Gasnikova, ${ }^{45}$ C. Gatti, ${ }^{50}$ A. Gaudiello, ${ }^{53 a, 53 b}$ G. Gaudio, ${ }^{123 a}$ I. L. Gavrilenko, ${ }^{98}$ C. Gay, ${ }^{171}$ G. Gaycken, ${ }^{23}$ E. N. Gazis, ${ }^{10}$ C. N. P. Gee, ${ }^{133}$ J. Geisen, ${ }^{57}$ M. Geisen, ${ }^{86}$ M. P. Geisler, ${ }^{60 a}$ K. Gellerstedt, ${ }^{148 a, 148 b}$ C. Gemme, ${ }^{53 a}$ M. H. Genest ${ }^{58}$ C. Geng, ${ }^{92}$ S. Gentile, ${ }^{134 a, 134 b}$ C. Gentsos, ${ }^{156}$ S. George ${ }^{80}$ D. Gerbaudo, ${ }^{13}$ A. Gershon, ${ }^{155}$ G. Geßner, ${ }^{46}$ S. Ghasemi, ${ }^{143}$ M. Ghneimat, ${ }^{23}$ B. Giacobbe, ${ }^{22 a}$ S. Giagu, ${ }^{134 a, 134 b}$ N. Giangiacomi, ${ }^{22 a, 22 b}$ P. Giannetti, ${ }^{126 a, 126 b}$ S. M. Gibson, ${ }^{80}$ M. Gignac, ${ }^{171}$ M. Gilchriese, ${ }^{16}$ D. Gillberg, ${ }^{31}$ G. Gilles, ${ }^{178}$ D. M. Gingrich, ${ }^{3, e}$ N. Giokaris, ${ }^{9, a}$ M. P. Giordani, ${ }^{167 a, 167 c}$ F. M. Giorgi, ${ }^{22 a}$ P. F. Giraud ${ }^{138}$ P. Giromini, ${ }^{59}$ D. Giugni, ${ }^{94 a}$

F. Giuli, ${ }^{122}$ C. Giuliani, ${ }^{103}$ M. Giulini, ${ }^{60 b}$ B. K. Gjelsten, ${ }^{121}$ S. Gkaitatzis, ${ }^{156}$ I. Gkialas, ${ }^{9, t}$ E. L. Gkougkousis, ${ }^{139}$ P. Gkountoumis, ${ }^{10}$ L. K. Gladilin, ${ }^{101}$ C. Glasman, ${ }^{85}$ J. Glatzer, ${ }^{13}$ P. C. F. Glaysher, ${ }^{45}$ A. Glazov, ${ }^{45}$ M. Goblirsch-Kolb, ${ }^{25}$ J. Godlewski, ${ }^{42}$ S. Goldfarb, ${ }^{91}$ T. Golling, ${ }^{52}$ D. Golubkov, ${ }^{132}$ A. Gomes, ${ }^{128 a, 128 b, 128 d}$ R. Gonçalo, ${ }^{128 a}$ R. Goncalves Gama, ${ }^{26 a}$ J. Goncalves Pinto Firmino Da Costa, ${ }^{138}$ G. Gonella, ${ }^{51}$ L. Gonella, ${ }^{19}$ A. Gongadze, ${ }^{68}$ S. González de la Hoz, ${ }^{170}$ S. Gonzalez-Sevilla, ${ }^{52}$ L. Goossens, ${ }^{32}$ P. A. Gorbounov, ${ }^{99}$ H. A. Gordon, ${ }^{27}$ I. Gorelov, ${ }^{107}$ B. Gorini, ${ }^{32}$ E. Gorini, ${ }^{76 a, 76 b}$ A. Gorišek, ${ }^{78}$ A. T. Goshaw, ${ }^{48}$ C. Gössling, ${ }^{46}$ M. I. Gostkin, ${ }^{68}$ C. A. Gottardo ${ }^{23}$ C. R. Goudet, ${ }^{119}$ D. Goujdami, ${ }^{137 c}$ A. G. Goussiou, ${ }^{140}$ N. Govender, ${ }^{147 b, u}$ E. Gozani, ${ }^{154}$ L. Graber,${ }^{57}$ I. Grabowska-Bold, ${ }^{41 a}$ P. O. J. Gradin, ${ }^{168}$ J. Gramling, ${ }^{166}$ E. Gramstad, ${ }^{121}$ S. Grancagnolo, ${ }^{17}$ V. Gratchev, ${ }^{125}$ P. M. Gravila, ${ }^{28 f}$ C. Gray, ${ }^{56}$ H. M. Gray, ${ }^{16}$ Z. D. Greenwood, ${ }^{82, v}$ 
C. Grefe, ${ }^{23}$ K. Gregersen, ${ }^{81}$ I. M. Gregor ${ }^{45}$ P. Grenier, ${ }^{145}$ K. Grevtsov, ${ }^{5}$ J. Griffiths, ${ }^{8}$ A. A. Grillo, ${ }^{139}$ K. Grimm, ${ }^{75}$ S. Grinstein, ${ }^{13, w}$ Ph. Gris, ${ }^{37}$ J.-F. Grivaz, ${ }^{119}$ S. Groh ${ }^{86}$ E. Gross, ${ }^{175}$ J. Grosse-Knetter,${ }^{57}$ G. C. Grossi,${ }^{82}$ Z. J. Grout ${ }^{81}$ A. Grummer, ${ }^{107}$ L. Guan, ${ }^{92}$ W. Guan, ${ }^{176}$ J. Guenther, ${ }^{65}$ F. Guescini, ${ }^{163 a}$ D. Guest, ${ }^{166}$ O. Gueta, ${ }^{155}$ B. Gui, ${ }^{113}$ E. Guido,${ }^{53 a, 53 b}$ T. Guillemin, ${ }^{5}$ S. Guindon, ${ }^{2}$ U. Gul, ${ }^{56}$ C. Gumpert, ${ }^{32}$ J. Guo, ${ }^{36 c}$ W. Guo, ${ }^{92}$ Y. Guo, ${ }^{36 a}$ R. Gupta, ${ }^{43}$ S. Gupta, ${ }^{122}$ G. Gustavino, ${ }^{134 a, 134 b}$ P. Gutierrez, ${ }^{115}$ N. G. Gutierrez Ortiz,${ }^{81}$ C. Gutschow,${ }^{81}$ C. Guyot, ${ }^{138}$ M. P. Guzik,${ }^{41 a}$ C. Gwenlan, ${ }^{122}$ C. B. Gwilliam, ${ }^{77}$ A. Haas, ${ }^{112}$ C. Haber,${ }^{16}$ H. K. Hadavand, ${ }^{8}$ N. Haddad, ${ }^{137 e}$ A. Hadef, ${ }^{88}$ S. Hageböck, ${ }^{23}$ M. Hagihara, ${ }^{164}$ H. Hakobyan, ${ }^{180, a}$ M. Haleem, ${ }^{45}$ J. Haley, ${ }^{16}$ G. Halladjian, ${ }^{93}$ G. D. Hallewell, ${ }^{88}$ K. Hamacher, ${ }^{178}$ P. Hamal, ${ }^{117}$ K. Hamano, ${ }^{172}$ A. Hamilton, ${ }^{147 a}$ G. N. Hamity, ${ }^{141}$ P. G. Hamnett, ${ }^{45}$ L. Han, ${ }^{36 a}$ S. Han, ${ }^{35 a}$ K. Hanagaki, ${ }^{69, x}$ K. Hanawa, ${ }^{157}$ M. Hance, ${ }^{139}$ B. Haney, ${ }^{124}$ P. Hanke, ${ }^{60 a}$ J. B. Hansen, ${ }^{39}$ J. D. Hansen, ${ }^{39}$ M. C. Hansen,${ }^{23}$ P. H. Hansen, ${ }^{39}$ K. Hara, ${ }^{164}$ A. S. Hard, ${ }^{176}$ T. Harenberg, ${ }^{178}$ F. Hariri, ${ }^{119}$ S. Harkusha, ${ }^{95}$ R. D. Harrington, ${ }^{49}$ P. F. Harrison, ${ }^{173}$ N. M. Hartmann, ${ }^{102}$ M. Hasegawa, ${ }^{70}$ Y. Hasegawa, ${ }^{142}$ A. Hasib, ${ }^{49}$ S. Hassani, ${ }^{138}$ S. Haug, ${ }^{18}$ R. Hauser, ${ }^{93}$ L. Hauswald, ${ }^{47}$ L. B. Havener, ${ }^{38}$ M. Havranek, ${ }^{130}$ C. M. Hawkes, ${ }^{19}$ R. J. Hawkings,${ }^{32}$ D. Hayakawa, ${ }^{159}$ D. Hayden,${ }^{93}$ C. P. Hays, ${ }^{122}$ J. M. Hays,${ }^{79}$ H. S. Hayward, ${ }^{77}$ S. J. Haywood, ${ }^{133}$ S. J. Head, ${ }^{19}$ T. Heck ${ }^{86}$ V. Hedberg, ${ }^{84}$ L. Heelan, ${ }^{8}$ S. Heer, ${ }^{23}$ K. K. Heidegger,${ }^{51}$ S. Heim, ${ }^{45}$ T. Heim ${ }^{16}$ B. Heinemann, ${ }^{45, y}$ J. J. Heinrich, ${ }^{102}$ L. Heinrich,${ }^{12}$ C. Heinz ${ }^{55}$ J. Hejbal, ${ }^{129}$ L. Helary, ${ }^{32}$ A. Held, ${ }^{171}$ S. Hellman, ${ }^{148 a, 148 b}$ C. Helsens, ${ }^{32}$ R. C. W. Henderson, ${ }^{75}$ Y. Heng, ${ }^{176}$ S. Henkelmann, ${ }^{171}$ A. M. Henriques Correia, ${ }^{32}$ S. Henrot-Versille, ${ }^{119}$ G. H. Herbert, ${ }^{17}$ H. Herde ${ }^{25}$ V. Herget, ${ }^{177}$ Y. Hernández Jiménez, ${ }^{147 \mathrm{c}}$ H. Herr, ${ }^{86}$ G. Herten, ${ }^{51}$ R. Hertenberger, ${ }^{102}$ L. Hervas, ${ }^{32}$ T. C. Herwig, ${ }^{124}$ G. G. Hesketh, ${ }^{81}$ N. P. Hessey, ${ }^{163 a}$ J. W. Hetherly, ${ }^{43}$ S. Higashino, ${ }^{69}$ E. Higón-Rodriguez, ${ }^{170}$ K. Hildebrand ${ }^{33}$ E. Hill, ${ }^{172}$ J. C. Hill, ${ }^{30}$ K. H. Hiller ${ }^{45}$ S. J. Hillier, ${ }^{19}$ M. Hils,${ }^{47}$ I. Hinchliffe, ${ }^{16}$ M. Hirose,${ }^{51}$ D. Hirschbuehl, ${ }^{178}$ B. Hiti, ${ }^{78}$ O. Hladik, ${ }^{129}$ X. Hoad,${ }^{49}$ J. Hobbs, ${ }^{150}$ N. Hod, ${ }^{163 a}$ M. C. Hodgkinson, ${ }^{141}$ P. Hodgson, ${ }^{141}$ A. Hoecker, ${ }^{32}$ M. R. Hoeferkamp, ${ }^{107}$ F. Hoenig, ${ }^{102}$ D. Hohn, ${ }^{23}$ T. R. Holmes,${ }^{33}$ M. Homann, ${ }^{46}$ S. Honda, ${ }^{164}$ T. Honda, ${ }^{69}$ T. M. Hong, ${ }^{127}$ B. H. Hooberman, ${ }^{169}$ W. H. Hopkins, ${ }^{118}$ Y. Horii, ${ }^{105}$ A. J. Horton, ${ }^{144}$ J-Y. Hostachy, ${ }^{58}$ S. Hou, ${ }^{153}$ A. Hoummada, ${ }^{137 a}$ J. Howarth, ${ }^{87}$ J. Hoya ${ }^{74}$ M. Hrabovsky, ${ }^{117}$ J. Hrdinka, ${ }^{32}$ I. Hristova, ${ }^{17}$ J. Hrivnac, ${ }^{119}$ T. Hryn'ova, ${ }^{5}$ A. Hrynevich, ${ }^{96}$ P. J. Hsu, ${ }^{63}$ S.-C. Hsu, ${ }^{140}$ Q. Hu, ${ }^{36 a}$ S. Hu, ${ }^{36 c}$ Y. Huang, ${ }^{35 a}$ Z. Hubacek, ${ }^{130}$ F. Hubaut, ${ }^{88}$ F. Huegging, ${ }^{23}$ T. B. Huffman, ${ }^{122}$ E. W. Hughes,${ }^{38}$ G. Hughes,${ }^{75}$ M. Huhtinen, ${ }^{32}$ P. Huo,${ }^{150}$ N. Huseynov, ${ }^{68, \mathrm{c}}$ J. Huston, ${ }^{93}$ J. Huth ${ }^{59}$ G. Iacobucci, ${ }^{52}$ G. Iakovidis, ${ }^{27}$ I. Ibragimov, ${ }^{143}$ L. Iconomidou-Fayard, ${ }^{119}$ Z. Idrissi, ${ }^{137 e}$ P. Iengo, ${ }^{32}$ O. Igonkina, ${ }^{109, z}$ T. Iizawa, ${ }^{174}$ Y. Ikegami, ${ }^{69}$ M. Ikeno, ${ }^{69}$ Y. Ilchenko, ${ }^{11, a a}$ D. Iliadis, ${ }^{156}$ N. Ilic, ${ }^{145}$ G. Introzzi, ${ }^{123 a, 123 b}$ P. Ioannou, ${ }^{9, a}$ M. Iodice, ${ }^{136 a} \mathrm{~K}$. Iordanidou, ${ }^{38} \mathrm{~V}$. Ippolito, ${ }^{59} \mathrm{M}$. F. Isacson, ${ }^{168} \mathrm{~N}$. Ishijima, ${ }^{120} \mathrm{M}$. Ishino, ${ }^{157} \mathrm{M}$. Ishitsuka, ${ }^{159} \mathrm{C}$. Issever, ${ }^{122}$ S. Istin, ${ }^{20 a}$ F. Ito, ${ }^{164} \mathrm{~J}$. M. Iturbe Ponce, ${ }^{62 a}$ R. Iuppa, ${ }^{162 a, 162 b}$ H. Iwasaki, ${ }^{69} \mathrm{~J}$. M. Izen, ${ }^{44}$ V. Izzo, ${ }^{106 a} \mathrm{~S}$. Jabbar, ${ }^{3}$ P. Jackson, ${ }^{1}$ R. M. Jacobs, ${ }^{23}$ V. Jain, ${ }^{2}$ K. B. Jakobi,${ }^{86}$ K. Jakobs ${ }^{51}$ S. Jakobsen, ${ }^{65}$ T. Jakoubek, ${ }^{129}$ D. O. Jamin, ${ }^{116}$ D. K. Jana, ${ }^{82}$ R. Jansky, ${ }^{52}$ J. Janssen, ${ }^{23}$ M. Janus ${ }^{57}$ P. A. Janus, ${ }^{41 a}$ G. Jarlskog, ${ }^{84}$ N. Javadov, ${ }^{68, c}$ T. Javůrek, ${ }^{51}$ M. Javurkova, ${ }^{51}$ F. Jeanneau, ${ }^{138}$ L. Jeanty, ${ }^{16}$ J. Jejelava, ${ }^{54 a, b b}$ A. Jelinskas, ${ }^{173}$ P. Jenni,,${ }^{51, c c}$ C. Jeske, ${ }^{173}$ S. Jézéquel, ${ }^{5}$ H. Ji, ${ }^{176}$ J. Jia, ${ }^{150}$ H. Jiang, ${ }^{67}$ Y. Jiang, ${ }^{36 \mathrm{a}}$ Z. Jiang, ${ }^{145}$ S. Jiggins, ${ }^{81}$ J. Jimenez Pena, ${ }^{170}$ S. Jin, ${ }^{35 \mathrm{a}}$ A. Jinaru, ${ }^{28 \mathrm{~b}}$ O. Jinnouchi, ${ }^{159}$ H. Jivan, ${ }^{147 \mathrm{c}}$ P. Johansson, ${ }^{141}$ K. A. Johns, ${ }^{7}$ C. A. Johnson, ${ }^{64}$ W. J. Johnson, ${ }^{140}$ K. Jon-And, ${ }^{148 a, 148 b}$ R. W. L. Jones, ${ }^{75}$ S. D. Jones, ${ }^{151}$ S. Jones, ${ }^{7}$ T. J. Jones,${ }^{77}$ J. Jongmanns, ${ }^{60 \mathrm{a}}$ P. M. Jorge, ${ }^{128 \mathrm{a}, 128 \mathrm{~b}}$ J. Jovicevic, ${ }^{163 \mathrm{a}}$ X. Ju, ${ }^{176}$ A. Juste Rozas, ${ }^{13, w}$ M. K. Köhler, ${ }^{175}$ A. Kaczmarska, ${ }^{42}$ M. Kado, ${ }^{119}$ H. Kagan, ${ }^{113}$ M. Kagan, ${ }^{145}$ S. J. Kahn,${ }^{88}$ T. Kaji, ${ }^{174}$ E. Kajomovitz, ${ }^{48}$ C. W. Kalderon, ${ }^{84}$ A. Kaluza, ${ }^{86}$ S. Kama, ${ }^{43}$ A. Kamenshchikov, ${ }^{132}$ N. Kanaya,${ }^{157}$ L. Kanjir, ${ }^{78}$ V. A. Kantserov, ${ }^{100}$ J. Kanzaki, ${ }^{69}$ B. Kaplan, ${ }^{112}$ L. S. Kaplan, ${ }^{176}$ D. Kar, ${ }^{147 \mathrm{c}}$ K. Karakostas, ${ }^{10}$ N. Karastathis, ${ }^{10}$ M. J. Kareem, ${ }^{57}$ E. Karentzos, ${ }^{10}$ S. N. Karpov, ${ }^{68}$ Z. M. Karpova ${ }^{68}$ K. Karthik, ${ }^{112}$ V. Kartvelishvili, ${ }^{75}$ A. N. Karyukhin, ${ }^{132}$ K. Kasahara, ${ }^{164}$ L. Kashif, ${ }^{176}$ R. D. Kass ${ }^{113}$ A. Kastanas, ${ }^{149}$ Y. Kataoka, ${ }^{157}$ C. Kato,${ }^{157}$ A. Katre,${ }^{52}$ J. Katzy, ${ }^{45}$ K. Kawade, ${ }^{70}$ K. Kawagoe, ${ }^{73}$ T. Kawamoto, ${ }^{157}$ G. Kawamura, ${ }^{57}$ E. F. Kay, ${ }^{77}$ V. F. Kazanin, ${ }^{111, d}$ R. Keeler, ${ }^{172}$ R. Kehoe, ${ }^{43}$ J. S. Keller, ${ }^{31}$ J. J. Kempster ${ }^{80}$ J Kendrick, ${ }^{19}$ H. Keoshkerian, ${ }^{161}$ O. Kepka, ${ }^{129}$ B. P. Kerševan, ${ }^{78}$ S. Kersten, ${ }^{178}$ R. A. Keyes, ${ }^{90}$ M. Khader, ${ }^{169}$ F. Khalil-zada, ${ }^{12}$ A. Khanov, ${ }^{116}$ A. G. Kharlamov, ${ }^{111, d}$ T. Kharlamova, ${ }^{111, d}$ A. Khodinov, ${ }^{160}$ T. J. Khoo, ${ }^{52}$ V. Khovanskiy, ${ }^{99, a}$ E. Khramov, ${ }^{68}$ J. Khubua, ${ }^{54 b, d d}$ S. Kido, ${ }^{70}$ C. R. Kilby, ${ }^{80}$ H. Y. Kim, ${ }^{8}$ S. H. Kim, ${ }^{164}$ Y. K. Kim, ${ }^{33}$ N. Kimura, ${ }^{156}$ O. M. Kind, ${ }^{17}$ B. T. King,${ }^{77}$ D. Kirchmeier, ${ }^{47}$ J. Kirk, ${ }^{133}$ A. E. Kiryunin, ${ }^{103}$ T. Kishimoto, ${ }^{157}$ D. Kisielewska, ${ }^{41 a}$ V. Kitali, ${ }^{45}$ K. Kiuchi, ${ }^{164}$ O. Kivernyk, ${ }^{5}$ E. Kladiva, ${ }^{146 \mathrm{~b}}$ T. Klapdor-Kleingrothaus, ${ }^{51}$ M. H. Klein, ${ }^{38}$ M. Klein, ${ }^{77}$ U. Klein, ${ }^{77}$ K. Kleinknecht,${ }^{86}$ P. Klimek, ${ }^{110}$ A. Klimentov, ${ }^{27}$ R. Klingenberg, ${ }^{46}$ T. Klingl, ${ }^{23}$ T. Klioutchnikova, ${ }^{32}$ E.-E. Kluge, ${ }^{60 a}$ P. Kluit, ${ }^{109}$ S. Kluth,${ }^{103}$ E. Kneringer, ${ }^{65}$ E. B. F. G. Knoops, ${ }^{88}$ A. Knue, ${ }^{103}$ A. Kobayashi, ${ }^{157}$ D. Kobayashi, ${ }^{159}$ T. Kobayashi, ${ }^{157}$ M. Kobel,${ }^{47}$ M. Kocian, ${ }^{145}$ P. Kodys, ${ }^{131}$ T. Koffas, ${ }^{31}$ E. Koffeman, ${ }^{109}$ N. M. Köhler, ${ }^{103}$ T. Koi, ${ }^{145}$ M. Kolb, ${ }^{60 b}$ I. Koletsou, ${ }^{5}$ A. A. Komar, ${ }^{98, a}$ Y. Komori, ${ }^{157}$ T. Kondo, ${ }^{69}$ N. Kondrashova, ${ }^{36 c}$ K. Köneke, ${ }^{51}$ A. C. König, ${ }^{108}$ T. Kono, ${ }^{69, \text { ee }}$ R. Konoplich, ${ }^{112, f f}$ 
N. Konstantinidis, ${ }^{81}$ R. Kopeliansky, ${ }^{64}$ S. Koperny, ${ }^{41 a}$ A. K. Kopp,${ }^{51}$ K. Korcyl,${ }^{42}$ K. Kordas, ${ }^{156}$ A. Korn, ${ }^{81}$ A. A. Korol, ${ }^{11, d}$ I. Korolkov, ${ }^{13}$ E. V. Korolkova, ${ }^{141}$ O. Kortner, ${ }^{103}$ S. Kortner, ${ }^{103}$ T. Kosek, ${ }^{131}$ V. V. Kostyukhin, ${ }^{23}$ A. Kotwal, ${ }^{48}$ A. Koulouris, ${ }^{10}$ A. Kourkoumeli-Charalampidi, ${ }^{123 a, 123 b}$ C. Kourkoumelis, ${ }^{9}$ E. Kourlitis, ${ }^{141}$ V. Kouskoura, ${ }^{27}$ A. B. Kowalewska, ${ }^{42}$ R. Kowalewski, ${ }^{172}$ T. Z. Kowalski, ${ }^{41 a}$ C. Kozakai, ${ }^{157}$ W. Kozanecki, ${ }^{138}$ A. S. Kozhin, ${ }^{132}$ V. A. Kramarenko, ${ }^{101}$ G. Kramberger ${ }^{78}$ D. Krasnopevtsev, ${ }^{100}$ M. W. Krasny, ${ }^{83}$ A. Krasznahorkay, ${ }^{32}$ D. Krauss, ${ }^{103}$ J. A. Kremer, ${ }^{41 a}$ J. Kretzschmar, ${ }^{77}$ K. Kreutzfeldt, ${ }^{55}$ P. Krieger, ${ }^{161}$ K. Krizka, ${ }^{33}$ K. Kroeninger, ${ }^{46}$ H. Kroha, ${ }^{103}$ J. Kroll, ${ }^{129}$ J. Kroll, ${ }^{124}$ J. Kroseberg, ${ }^{23}$ J. Krstic, ${ }^{14}$ U. Kruchonak, ${ }^{68}$ H. Krüger, ${ }^{23}$ N. Krumnack, ${ }^{67}$ M. C. Kruse, ${ }^{48}$ T. Kubota, ${ }^{91}$ H. Kucuk, ${ }^{81}$ S. Kuday, ${ }^{46}$ J. T. Kuechler, ${ }^{178}$ S. Kuehn, ${ }^{32}$ A. Kugel, ${ }^{60 a}$ F. Kuger, ${ }^{177}$ T. Kuhl, ${ }^{45}$ V. Kukhtin, ${ }^{68}$ R. Kukla, ${ }^{88}$ Y. Kulchitsky, ${ }^{95}$ S. Kuleshov, ${ }^{34 \mathrm{~b}}$ Y. P. Kulinich, ${ }^{169}$ M. Kuna, ${ }^{14 \mathrm{a}, 134 \mathrm{~b}}$ T. Kunigo, ${ }^{71}$ A. Kupco, ${ }^{129}$ T. Kupfer, ${ }^{46}$ O. Kuprash, ${ }^{155}$ H. Kurashige, ${ }^{70}$ L. L. Kurchaninov, ${ }^{163}$ Y. A. Kurochkin, ${ }^{95}$ M. G. Kurth, ${ }^{35 a}$ V. Kus, ${ }^{129}$ E. S. Kuwertz, ${ }^{172}$ M. Kuze, ${ }^{159}$ J. Kvita, ${ }^{117}$ T. Kwan, ${ }^{172}$ D. Kyriazopoulos, ${ }^{141}$ A. La Rosa, ${ }^{103}$ J. L. La Rosa Navarro, ${ }^{26 \mathrm{~d}}$ L. La Rotonda, ${ }^{40,40 \mathrm{~b}}$ F. La Ruffa ${ }^{40 \mathrm{a}, 40 \mathrm{~b}}$ C. Lacasta, ${ }^{170}$ F. Lacava, ${ }^{134 a, 134 \mathrm{~b}}$ J. Lacey, ${ }^{45}$ H. Lacker, ${ }^{17}$ D. Lacour, ${ }^{83}$ E. Ladygin, ${ }^{68}$ R. Lafaye, ${ }^{5}$ B. Laforge, ${ }^{83}$ T. Lagouri, ${ }^{179}$ S. Lai, ${ }^{57}$ S. Lammers, ${ }^{64}$ W. Lampl, ${ }^{7}$ E. Lançon, ${ }^{27}$ U. Landgraf, ${ }^{51}$ M. P. J. Landon, ${ }^{79}$ M. C. Lanfermann, ${ }^{52}$ V. S. Lang, ${ }^{60 a}$ J. C. Lange, ${ }^{13}$ R. J. Langenberg, ${ }^{32}$ A. J. Lankford, ${ }^{166}$ F. Lanni, ${ }^{27}$ K. Lantzsch, ${ }^{23}$ A. Lanza, ${ }^{123 a}$ A. Lapertosa, ${ }^{53 a, 53 b}$ S. Laplace, ${ }^{83}$ J. F. Laporte, ${ }^{138}$ T. Lari, ${ }^{94 a}$ F. Lasagni Manghi, ${ }^{22 a, 22 b}$ M. Lassnig, ${ }^{32}$

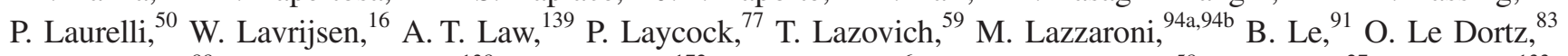
E. Le Guirriec, ${ }^{88}$ E. P. Le Quilleuc, ${ }^{138}$ M. LeBlanc, ${ }^{172}$ T. LeCompte,${ }^{6}$ F. Ledroit-Guillon, ${ }^{58}$ C. A. Lee,${ }^{27}$ G. R. Lee, ${ }^{133, g g}$ S. C. Lee ${ }^{153}$ L. Lee ${ }^{59}$ B. Lefebvre, ${ }^{90}$ G. Lefebvre, ${ }^{83}$ M. Lefebvre, ${ }^{172}$ F. Legger, ${ }^{102}$ C. Leggett,,${ }^{16}$ G. Lehmann Miotto, ${ }^{32}$ X. Lei, ${ }^{7}$ W. A. Leight, ${ }^{45}$ M. A. L. Leite, ${ }^{26 d}$ R. Leitner, ${ }^{131}$ D. Lellouch, ${ }^{175}$ B. Lemmer, ${ }^{57}$ K. J. C. Leney, ${ }^{81}$ T. Lenz, ${ }^{23}$ B. Lenzi, ${ }^{32}$ R. Leone, ${ }^{7}$ S. Leone, ${ }^{126 a, 126 b}$ C. Leonidopoulos, ${ }^{49}$ G. Lerner, ${ }^{151}$ C. Leroy, ${ }^{97}$ A. A. J. Lesage, ${ }^{138}$ C. G. Lester, ${ }^{30}$ M. Levchenko, ${ }^{125}$ J. Levêque, ${ }^{5}$ D. Levin, ${ }^{92}$ L. J. Levinson, ${ }^{175}$ M. Levy, ${ }^{19}$ D. Lewis, ${ }^{79}$ B. Li,${ }^{36 a, h h}$ Changqiao Li, ${ }^{36 a} \mathrm{H} . \mathrm{Li}^{1}{ }^{150}$ L. Li, ${ }^{36 \mathrm{c}}$ Q. $\mathrm{Li},{ }^{35 \mathrm{a}} \mathrm{S} . \mathrm{Li},{ }^{48}$ X. Li, ${ }^{36 \mathrm{c}}$ Y. Li, ${ }^{143}$ Z. Liang, ${ }^{35 \mathrm{a}}$ B. Liberti, ${ }^{135 \mathrm{a}}$ A. Liblong, ${ }^{161} \mathrm{~K}$. Lie, ${ }^{62 \mathrm{c}}$ J. Liebal, ${ }^{23}$ W. Liebig, ${ }^{15}$ A. Limosani, ${ }^{152}$ S. C. Lin ${ }^{182}$ T. H. Lin,${ }^{86}$ B. E. Lindquist, ${ }^{150}$ A. E. Lionti, ${ }^{52}$ E. Lipeles, ${ }^{124}$ A. Lipniacka, ${ }^{15}$ M. Lisovyi, ${ }^{60 b}$ T. M. Liss, ${ }^{169, i i}$ A. Lister, ${ }^{171}$ A. M. Litke, ${ }^{139}$ B. Liu, ${ }^{153, j \mathrm{j}}$ H. Liu, ${ }^{92}$ H. Liu, ${ }^{27}$ J. K. K. Liu, ${ }^{122}$ J. Liu, ${ }^{36 \mathrm{~b}}$ J. B. Liu, ${ }^{36 \mathrm{a}}$ K. Liu, ${ }^{88}$ L. Liu, ${ }^{169}$ M. Liu, ${ }^{36 a}$ Y. L. Liu, ${ }^{36 a}$ Y. Liu, ${ }^{36 a}$ M. Livan, ${ }^{123 a, 123 b}$ A. Lleres, ${ }^{58}$ J. Llorente Merino, ${ }^{35 a}$ S. L. Lloyd ${ }^{79}$ C. Y. Lo, ${ }^{62 b}$ F. Lo Sterzo, ${ }^{153}$ E. M. Lobodzinska, ${ }^{45}$ P. Loch, ${ }^{7}$ F. K. Loebinger,${ }^{87}$ A. Loesle, ${ }^{51}$ K. M. Loew, ${ }^{25}$ A. Loginov, ${ }^{179, a}$ T. Lohse, ${ }^{17}$

K. Lohwasser, ${ }^{141}$ M. Lokajicek, ${ }^{129}$ B. A. Long, ${ }^{24}$ J. D. Long, ${ }^{169}$ R. E. Long, ${ }^{75}$ L. Longo, ${ }^{76 a, 76 b}$ K. A. Looper, ${ }^{113}$ J. A. Lopez, ${ }^{34 b}$ D. Lopez Mateos ${ }^{59}$ I. Lopez Paz, ${ }^{13}$ A. Lopez Solis ${ }^{83}$ J. Lorenz, ${ }^{102}$ N. Lorenzo Martinez,${ }^{5}$ M. Losada, ${ }^{21}$ P. J. Lösel, ${ }^{102}$ X. Lou, ${ }^{35 a}$ A. Lounis, ${ }^{119}$ J. Love, ${ }^{6}$ P. A. Love, ${ }^{75}$ H. Lu, ${ }^{62 a}$ N. Lu, ${ }^{92}$ Y. J. Lu, ${ }^{63}$ H. J. Lubatti, ${ }^{140}$ C. Luci, ${ }^{134 a, 134 b}$ A. Lucotte,${ }^{58}$ C. Luedtke, ${ }^{51}$ F. Luehring, ${ }^{64}$ W. Lukas, ${ }^{65}$ L. Luminari, ${ }^{134 a}$ O. Lundberg, ${ }^{148 a, 148 b}$ B. Lund-Jensen, ${ }^{149}$ M. S. Lutz ${ }^{89}$ P. M. Luzi ${ }^{83}$ D. Lynn, ${ }^{27}$ R. Lysak, ${ }^{129}$ E. Lytken, ${ }^{84}$ F. Lyu, ${ }^{35 a}$ V. Lyubushkin, ${ }^{68}$ H. Ma, ${ }^{27}$ L. L. Ma,${ }^{36 b}$ Y. Ma,${ }^{36 b}$ G. Maccarrone, ${ }^{50}$ A. Macchiolo, ${ }^{103}$ C. M. Macdonald, ${ }^{141}$ B. Maček, ${ }^{78}$ J. Machado Miguens, ${ }^{124,128 b}$ D. Madaffari, ${ }^{170}$ R. Madar, ${ }^{37}$ W. F. Mader ${ }^{47}$ A. Madsen, ${ }^{45}$ J. Maeda, ${ }^{70}$ S. Maeland, ${ }^{15}$ T. Maeno, ${ }^{27}$ A. S. Maevskiy, ${ }^{101}$ V. Magerl, ${ }^{51}$ J. Mahlstedt, ${ }^{109}$ C. Maiani, ${ }^{119}$ C. Maidantchik, ${ }^{26 a}$ A. A. Maier, ${ }^{103}$ T. Maier, ${ }^{102}$ A. Maio, ${ }^{128 a, 128 b, 128 d}$ O. Majersky, ${ }^{146 a}$ S. Majewski, ${ }^{118}$ Y. Makida, ${ }^{69}$ N. Makovec, ${ }^{119}$ B. Malaescu, ${ }^{83}$ Pa. Malecki, ${ }^{42}$ V. P. Maleev, ${ }^{125}$ F. Malek, ${ }^{58}$ U. Mallik, ${ }^{66}$ D. Malon, ${ }^{6}$ C. Malone, ${ }^{30}$ S. Maltezos, ${ }^{10}$ S. Malyukov, ${ }^{32}$ J. Mamuzic, ${ }^{170}$ G. Mancini, ${ }^{50}$ I. Mandić, ${ }^{78}$ J. Maneira, ${ }^{128 a, 128 b}$ L. Manhaes de Andrade Filho, ${ }^{26 \mathrm{~b}}$ J. Manjarres Ramos, ${ }^{47}$ K. H. Mankinen, ${ }^{84}$ A. Mann, ${ }^{102}$ A. Manousos, ${ }^{32}$ B. Mansoulie, ${ }^{138}$ J. D. Mansour, ${ }^{35 a}$ R. Mantifel, ${ }^{90}$ M. Mantoani, ${ }^{57}$ S. Manzoni, ${ }^{94 a, 94 b}$ L. Mapelli, ${ }^{32}$ G. Marceca,${ }^{29}$ L. March, ${ }^{52}$ L. Marchese, ${ }^{122}$ G. Marchiori, ${ }^{83}$ M. Marcisovsky, ${ }^{129}$ M. Marjanovic, ${ }^{37}$ D. E. Marley, ${ }^{92}$ F. Marroquim, ${ }^{26 a}$ S. P. Marsden, ${ }^{87}$ Z. Marshall, ${ }^{16}$ M. U. F Martensson, ${ }^{168}$ S. Marti-Garcia, ${ }^{170}$ C. B. Martin, ${ }^{113}$ T. A. Martin, ${ }^{173}$ V. J. Martin, ${ }^{49}$ B. Martin dit Latour, ${ }^{15}$ M. Martinez, ${ }^{13, w}$ V. I. Martinez Outschoorn, ${ }^{169}$ S. Martin-Haugh, ${ }^{133}$ V. S. Martoiu, ${ }^{28 b}$ A. C. Martyniuk, ${ }^{81}$ A. Marzin, ${ }^{32}$ L. Masetti, ${ }^{86}$ T. Mashimo, ${ }^{157}$ R. Mashinistov, ${ }^{98}$ J. Masik, ${ }^{87}$ A. L. Maslennikov, ${ }^{111, d}$ L. Massa, ${ }^{135 a, 135 b}$ P. Mastrandrea, ${ }^{5}$ A. Mastroberardino, ${ }^{40 a, 40 b}$ T. Masubuchi, ${ }^{157}$ P. Mättig, ${ }^{178}$ J. Maurer ${ }^{28 b}$ S. J. Maxfield, ${ }^{77}$ D. A. Maximov, ${ }^{111, d}$ R. Mazini, ${ }^{153}$ I. Maznas, ${ }^{156}$ S. M. Mazza, ${ }^{94 a, 94 b}$ N. C. Mc Fadden, ${ }^{107}$ G. Mc Goldrick, ${ }^{161}$ S. P. Mc Kee, ${ }^{92}$ A. McCarn, ${ }^{92}$ R. L. McCarthy, ${ }^{150}$ T. G. McCarthy, ${ }^{103}$ L. I. McClymont, ${ }^{81}$ E. F. McDonald, ${ }^{91}$ J. A. Mcfayden, ${ }^{81}$ G. Mchedlidze,${ }^{57}$ S. J. McMahon, ${ }^{133}$ P. C. McNamara, ${ }^{91}$ R. A. McPherson, ${ }^{172, p}$ S. Meehan, ${ }^{140}$ T. J. Megy,${ }^{51}$ S. Mehlhase, ${ }^{102}$ A. Mehta, ${ }^{77}$ T. Meideck, ${ }^{58}$ K. Meier, ${ }^{60 \mathrm{a}}$ B. Meirose, ${ }^{44}$ D. Melini, ${ }^{170, k \mathrm{kk}}$ B. R. Mellado Garcia,${ }^{147 \mathrm{c}}$ J. D. Mellenthin, ${ }^{57}$ M. Melo, ${ }^{146 \mathrm{a}}$ F. Meloni, ${ }^{18}$ A. Melzer, ${ }^{23}$ S. B. Menary, ${ }^{87}$ L. Meng, ${ }^{77}$ X. T. Meng, ${ }^{92}$ A. Mengarelli, ${ }^{22 a, 22 b}$ S. Menke, ${ }^{103}$ E. Meoni, ${ }^{40 a, 40 b}$ S. Mergelmeyer, ${ }^{17}$ P. Mermod, ${ }^{52}$ L. Merola, ${ }^{106 a, 106 b}$ C. Meroni, ${ }^{94 a}$ F. S. Merritt, ${ }^{33}$ A. Messina, ${ }^{134 a, 134 b}$ J. Metcalfe, ${ }^{6}$ A. S. Mete, ${ }^{166}$ C. Meyer, ${ }^{124}$ 
J-P. Meyer, ${ }^{138}$ J. Meyer, ${ }^{109}$ H. Meyer Zu Theenhausen ${ }^{60 a}$ F. Miano, ${ }^{151}$ R. P. Middleton, ${ }^{133}$ S. Miglioranzi, ${ }^{53 a, 53 b}$ L. Mijović, ${ }^{49}$ G. Mikenberg, ${ }^{175}$ M. Mikestikova, ${ }^{129}$ M. Mikuž ${ }^{78}$ M. Milesi, ${ }^{91}$ A. Milic, ${ }^{161}$ D. W. Miller,${ }^{33}$ C. Mills, ${ }^{49}$ A. Milov, ${ }^{175}$

D. A. Milstead, ${ }^{148 a, 148 b}$ A. A. Minaenko, ${ }^{132}$ Y. Minami, ${ }^{157}$ I. A. Minashvili, ${ }^{68}$ A. I. Mincer ${ }^{112}$ B. Mindur ${ }^{41 a}$ M. Mineev, ${ }^{68}$ Y. Minegishi, ${ }^{157}$ Y. Ming, ${ }^{176}$ L. M. Mir, ${ }^{13}$ K. P. Mistry, ${ }^{124}$ T. Mitani, ${ }^{174}$ J. Mitrevski, ${ }^{102}$ V. A. Mitsou, ${ }^{170}$ A. Miucci, ${ }^{18}$

P. S. Miyagawa, ${ }^{141}$ A. Mizukami, ${ }^{69}$ J. U. Mjörnmark, ${ }^{84}$ T. Mkrtchyan, ${ }^{180}$ M. Mlynarikova, ${ }^{131}$ T. Moa, ${ }^{148 a, 148 b}$

K. Mochizuki, ${ }^{97}$ P. Mogg,${ }^{51}$ S. Mohapatra ${ }^{38}$ S. Molander, ${ }^{148 a, 148 b}$ R. Moles-Valls, ${ }^{23}$ R. Monden,${ }^{71}$ M. C. Mondragon, ${ }^{93}$ K. Mönig, ${ }^{45}$ J. Monk, ${ }^{39}$ E. Monnier, ${ }^{88}$ A. Montalbano, ${ }^{150}$ J. Montejo Berlingen, ${ }^{32}$ F. Monticelli, ${ }^{74}$ S. Monzani, ${ }^{94 a, 94 b}$ R. W. Moore, ${ }^{3}$ N. Morange, ${ }^{119}$ D. Moreno, ${ }^{21}$ M. Moreno Llácer, ${ }^{32}$ P. Morettini, ${ }^{53 a}$ S. Morgenstern, ${ }^{32}$ D. Mori, ${ }^{144}$ T. Mori, ${ }^{157}$

M. Morii, ${ }^{59}$ M. Morinaga, ${ }^{157}$ V. Morisbak, ${ }^{121}$ A. K. Morley, ${ }^{32}$ G. Mornacchi, ${ }^{32}$ J. D. Morris, ${ }^{79}$ L. Morvaj, ${ }^{150}$

P. Moschovakos, ${ }^{10}$ M. Mosidze,${ }^{54 b}$ H. J. Moss, ${ }^{141}$ J. Moss, ${ }^{145,11}$ K. Motohashi,${ }^{159}$ R. Mount, ${ }^{145}$ E. Mountricha, ${ }^{27}$

E. J. W. Moyse, ${ }^{89}$ S. Muanza, ${ }^{88}$ F. Mueller, ${ }^{103}$ J. Mueller ${ }^{127}$ R. S. P. Mueller, ${ }^{102}$ D. Muenstermann, ${ }^{75}$ P. Mullen, ${ }^{56}$

G. A. Mullier, ${ }^{18}$ F. J. Munoz Sanchez ${ }^{87}$ W. J. Murray, ${ }^{173,133}$ H. Musheghyan, ${ }^{32}$ M. Muškinja ${ }^{78}$ A. G. Myagkov, ${ }^{132, m m}$

M. Myska, ${ }^{130}$ B. P. Nachman, ${ }^{16}$ O. Nackenhorst,${ }^{52}$ K. Nagai, ${ }^{122}$ R. Nagai, ${ }^{69, e e}$ K. Nagano, ${ }^{69}$ Y. Nagasaka,${ }^{61}$ K. Nagata, ${ }^{164}$

M. Nagel, ${ }^{51}$ E. Nagy, ${ }^{88}$ A. M. Nairz, ${ }^{32}$ Y. Nakahama, ${ }^{105}$ K. Nakamura, ${ }^{69}$ T. Nakamura, ${ }^{157}$ I. Nakano, ${ }^{114}$

R. F. Naranjo Garcia, ${ }^{45}$ R. Narayan, ${ }^{11}$ D. I. Narrias Villar, ${ }^{60 a}$ I. Naryshkin, ${ }^{125}$ T. Naumann, ${ }^{45}$ G. Navarro, ${ }^{21}$ R. Nayyar, ${ }^{7}$ H. A. Neal,${ }^{92}$ P. Yu. Nechaeva, ${ }^{98}$ T. J. Neep,${ }^{138}$ A. Negri, ${ }^{123 a, 123 b}$ M. Negrini, ${ }^{22 a}$ S. Nektarijevic, ${ }^{108}$ C. Nellist, ${ }^{119}$ A. Nelson, ${ }^{166}$ M. E. Nelson, ${ }^{122}$ S. Nemecek, ${ }^{129}$ P. Nemethy, ${ }^{112}$ M. Nessi, ${ }^{32, n n}$ M. S. Neubauer, ${ }^{169}$ M. Neumann, ${ }^{178}$ P. R. Newman, ${ }^{19}$ T. Y. Ng, ${ }^{62 \mathrm{c}}$ T. Nguyen Manh,${ }^{97}$ R. B. Nickerson, ${ }^{122}$ R. Nicolaidou, ${ }^{138}$ J. Nielsen, ${ }^{139}$ V. Nikolaenko, ${ }^{132, m m}$ I. Nikolic-Audit, ${ }^{83}$ K. Nikolopoulos, ${ }^{19}$ J. K. Nilsen, ${ }^{121}$ P. Nilsson, ${ }^{27}$ Y. Ninomiya, ${ }^{157}$ A. Nisati, ${ }^{134 a}$ N. Nishu, ${ }^{35 c}$ R. Nisius, ${ }^{103}$ I. Nitsche, ${ }^{46}$ T. Nitta, ${ }^{174}$ T. Nobe, ${ }^{157}$ Y. Noguchi, ${ }^{71}$ M. Nomachi, ${ }^{120}$ I. Nomidis, ${ }^{31}$ M. A. Nomura, ${ }^{27}$ T. Nooney, ${ }^{79}$ M. Nordberg, ${ }^{32}$ N. Norjoharuddeen, ${ }^{122}$ O. Novgorodova, ${ }^{47}$ S. Nowak,${ }^{103}$ M. Nozaki, ${ }^{69}$ L. Nozka, ${ }^{117}$ K. Ntekas, ${ }^{166}$ E. Nurse,${ }^{81}$ F. Nuti, ${ }^{91}$ K. O'connor, ${ }^{25}$ D. C. O'Neil, ${ }^{144}$ A. A. O'Rourke, ${ }^{45}$ V. O'Shea, ${ }^{56}$ F. G. Oakham, ${ }^{31, e}$ H. Oberlack, ${ }^{103}$ T. Obermann, ${ }^{23}$ J. Ocariz, ${ }^{83}$ A. Ochi ${ }^{70}$ I. Ochoa, ${ }^{38}$ J. P. Ochoa-Ricoux ${ }^{34 a}$ S. Oda ${ }^{73}$ S. Odaka ${ }^{69}$ A. Oh ${ }^{87}$ S. H. Oh, ${ }^{48}$ C. C. Ohm, ${ }^{16}$ H. Ohman, ${ }^{168}$ H. Oide, ${ }^{53 a, 53 b}$ H. Okawa, ${ }^{164}$ Y. Okumura, ${ }^{157}$ T. Okuyama, ${ }^{69}$ A. Olariu, ${ }^{28 b}$ L. F. Oleiro Seabra, ${ }^{128 a}$ S. A. Olivares Pino, ${ }^{34 a}$ D. Oliveira Damazio, ${ }^{27}$ A. Olszewski, ${ }^{42}$ J. Olszowska, ${ }^{42}$ A. Onofre, ${ }^{128 a, 128 e}$ K. Onogi, ${ }^{105}$ P. U. E. Onyisi, ${ }^{11, a a}$ H. Oppen, ${ }^{121}$ M. J. Oreglia, ${ }^{33}$ Y. Oren, ${ }^{155}$ D. Orestano, ${ }^{136 a, 136 b}$ N. Orlando, ${ }^{62 b}$ R. S. Orr, ${ }^{161}$ B. Osculati, ${ }^{53 a, 53 b, a}$ R. Ospanov, ${ }^{36 a}$ G. Otero y Garzon, ${ }^{29}$ H. Otono,${ }^{73}$ M. Ouchrif, ${ }^{137 d}$ F. Ould-Saada, ${ }^{121}$ A. Ouraou, ${ }^{138}$ K. P. Oussoren, ${ }^{109}$ Q. Ouyang, ${ }^{35 a}$ M. Owen, ${ }^{56}$ R. E. Owen, ${ }^{19}$ V. E. Ozcan, ${ }^{20 a}$ N. Ozturk, ${ }^{8}$ K. Pachal, ${ }^{144}$ A. Pacheco Pages ${ }^{13}$ L. Pacheco Rodriguez, ${ }^{138}$ C. Padilla Aranda,${ }^{13}$ S. Pagan Griso,${ }^{16}$ M. Paganini,${ }^{179}$ F. Paige, ${ }^{27}$ G. Palacino, ${ }^{64}$ S. Palazzo, ${ }^{40 a, 40 b}$ S. Palestini, ${ }^{32}$ M. Palka, ${ }^{41 b}$ D. Pallin, ${ }^{37}$ E. St. Panagiotopoulou, ${ }^{10}$ I. Panagoulias, ${ }^{10}$ C. E. Pandini, ${ }^{126 a, 126 b}$ J. G. Panduro Vazquez, ${ }^{80}$ P. Pani, ${ }^{32}$ S. Panitkin, ${ }^{27}$ D. Pantea, ${ }^{28 b}$ L. Paolozzi, ${ }^{52}$ Th. D. Papadopoulou, ${ }^{10}$ K. Papageorgiou, ${ }^{9, t}$ A. Paramonov, ${ }^{6}$ D. Paredes Hernandez, ${ }^{179}$ A. J. Parker, ${ }^{75}$ M. A. Parker, ${ }^{30}$ K. A. Parker ${ }^{45}$ F. Parodi, ${ }^{53 a, 53 b}$ J. A. Parsons, ${ }^{38}$ U. Parzefall, ${ }^{51}$ V. R. Pascuzzi, ${ }^{161}$ J. M. Pasner ${ }^{139}$ E. Pasqualucci,${ }^{134 a}$ S. Passaggio,${ }^{53 a}$ Fr. Pastore ${ }^{80}$ S. Pataraia,${ }^{86}$ J. R. Pater ${ }^{87}$ T. Pauly,${ }^{32}$ B. Pearson, ${ }^{103}$ S. Pedraza Lopez,${ }^{170}$ R. Pedro, ${ }^{128 a, 128 b}$ S. V. Peleganchuk, ${ }^{111, d}$ O. Penc,${ }^{129}$ C. Peng, ${ }^{35 a}$ H. Peng, ${ }^{36 a}$

J. Penwell ${ }^{64}$ B. S. Peralva, ${ }^{26 \mathrm{~b}}$ M. M. Perego, ${ }^{138}$ D. V. Perepelitsa, ${ }^{27}$ F. Peri, ${ }^{17}$ L. Perini, ${ }^{94 a, 94 b}$ H. Pernegger, ${ }^{32}$ S. Perrella, ${ }^{106 a, 106 b}$ R. Peschke, ${ }^{45}$ V. D. Peshekhonov,${ }^{68, a}$ K. Peters,${ }^{45}$ R. F. Y. Peters, ${ }^{87}$ B. A. Petersen, ${ }^{32}$ T. C. Petersen, ${ }^{39}$ E. Petit ${ }^{58}$ A. Petridis, ${ }^{1}$ C. Petridou, ${ }^{156}$ P. Petroff, ${ }^{119}$ E. Petrolo, ${ }^{134 a}$ M. Petrov ${ }^{122}$ F. Petrucci, ${ }^{136 a, 136 b}$ N. E. Pettersson, ${ }^{89}$ A. Peyaud ${ }^{138}$ R. Pezoa, ${ }^{34 b}$ F. H. Phillips,${ }^{93}$ P. W. Phillips, ${ }^{133}$ G. Piacquadio, ${ }^{150}$ E. Pianori, ${ }^{173}$ A. Picazio,${ }^{89}$ E. Piccaro, ${ }^{79}$ M. A. Pickering, ${ }^{122}$ R. Piegaia, ${ }^{29}$ J. E. Pilcher, ${ }^{33}$ A. D. Pilkington, ${ }^{87}$ A. W. J. Pin, ${ }^{87}$ M. Pinamonti, ${ }^{135 a, 135 b}$ J. L. Pinfold, ${ }^{3}$ H. Pirumov, ${ }^{45}$ M. Pitt, ${ }^{175}$ L. Plazak, ${ }^{146 a}$ M.-A. Pleier,${ }^{27}$ V. Pleskot,${ }^{86}$ E. Plotnikova,${ }^{68}$ D. Pluth, ${ }^{67}$ P. Podberezko, ${ }^{111}$

R. Poettgen, ${ }^{148 a, 148 b}$ R. Poggi, ${ }^{123 a, 123 b}$ L. Poggioli, ${ }^{119}$ D. Pohl,${ }^{23}$ G. Polesello, ${ }^{123 a}$ A. Poley, ${ }^{45}$ A. Policicchio, ${ }^{40 a, 40 b}$ R. Polifka, ${ }^{32}$ A. Polini, ${ }^{22 a}$ C. S. Pollard, ${ }^{56}$ V. Polychronakos, ${ }^{27}$ K. Pommès, ${ }^{32}$ D. Ponomarenko, ${ }^{100}$ L. Pontecorvo, ${ }^{134 a}$ G. A. Popeneciu, ${ }^{28 d}$ A. Poppleton, ${ }^{32}$ S. Pospisil, ${ }^{130}$ K. Potamianos, ${ }^{16}$ I. N. Potrap,${ }^{68}$ C. J. Potter, ${ }^{30}$ G. Poulard ${ }^{32}$ T. Poulsen,${ }^{84}$ J. Poveda ${ }^{32}$ M. E. Pozo Astigarraga, ${ }^{32}$ P. Pralavorio, ${ }^{88}$ A. Pranko, ${ }^{16}$ S. Prell, ${ }^{67}$ D. Price, ${ }^{87}$ M. Primavera, ${ }^{76 a}$ S. Prince, ${ }^{90}$ N. Proklova, ${ }^{100}$ K. Prokofiev, ${ }^{62 \mathrm{c}}$ F. Prokoshin, ${ }^{34 \mathrm{~b}}$ S. Protopopescu, ${ }^{27}$ J. Proudfoot, ${ }^{6}$ M. Przybycien, ${ }^{41 \mathrm{a}}$ A. Puri, ${ }^{169}$ P. Puzo, ${ }^{119}$ J. Qian, ${ }^{92}$ G. Qin, ${ }^{56}$ Y. Qin ${ }^{87}$ A. Quadt,${ }^{57}$ M. Queitsch-Maitland, ${ }^{45}$ D. Quilty, ${ }^{56}$ S. Raddum, ${ }^{121}$ V. Radeka, ${ }^{27}$ V. Radescu, ${ }^{122}$ S. K. Radhakrishnan ${ }^{150}$ P. Radloff, ${ }^{118}$ P. Rados, ${ }^{91}$ F. Ragusa, ${ }^{94 a, 94 b}$ G. Rahal,${ }^{181}$ J. A. Raine, ${ }^{87}$ S. Rajagopalan ${ }^{27}$ C. Rangel-Smith, ${ }^{168}$ T. Rashid, ${ }^{119}$ S. Raspopov, ${ }^{5}$ M. G. Ratti, ${ }^{94,94 b}$ D. M. Rauch, ${ }^{45}$ F. Rauscher, ${ }^{102}$ S. Rave,${ }^{86}$ I. Ravinovich, ${ }^{175}$ J. H. Rawling, ${ }^{87}$ M. Raymond, ${ }^{32}$ A. L. Read, ${ }^{121}$ N. P. Readioff, ${ }^{58}$ M. Reale,${ }^{76 a, 76 b}$ D. M. Rebuzzi, ${ }^{123 a, 123 b}$ 
A. Redelbach, ${ }^{177}$ G. Redlinger, ${ }^{27}$ R. Reece, ${ }^{139}$ R. G. Reed,${ }^{147 c}$ K. Reeves, ${ }^{44}$ L. Rehnisch, ${ }^{17}$ J. Reichert, ${ }^{124}$ A. Reiss, ${ }^{86}$ C. Rembser, ${ }^{32}$ H. Ren,${ }^{35 a}$ M. Rescigno, ${ }^{134 a}$ S. Resconi, ${ }^{94 a}$ E. D. Resseguie, ${ }^{124}$ S. Rettie, ${ }^{171}$ E. Reynolds, ${ }^{19}$ O. L. Rezanova, ${ }^{111, \mathrm{~d}}$ P. Reznicek, ${ }^{131}$ R. Rezvani, ${ }^{97}$ R. Richter, ${ }^{103}$ S. Richter, ${ }^{81}$ E. Richter-Was, ${ }^{41 \mathrm{~b}}$ O. Ricken, ${ }^{23}$ M. Ridel, ${ }^{83}$ P. Rieck, ${ }^{103}$ C. J. Riegel, ${ }^{178}$ J. Rieger,${ }^{57}$ O. Rifki, ${ }^{115}$ M. Rijssenbeek, ${ }^{150}$ A. Rimoldi, ${ }^{123 a, 123 b}$ M. Rimoldi, ${ }^{18}$ L. Rinaldi, ${ }^{22 a}$ G. Ripellino, ${ }^{149}$ B. Ristić, ${ }^{32}$ E. Ritsch, ${ }^{32}$ I. Riu, ${ }^{13}$ F. Rizatdinova, ${ }^{116}$ E. Rizvi, ${ }^{79}$ C. Rizzi, ${ }^{13}$ R. T. Roberts,${ }^{87}$

S. H. Robertson, ${ }^{90, p}$ A. Robichaud-Veronneau, ${ }^{90}$ D. Robinson, ${ }^{30}$ J. E. M. Robinson, ${ }^{45}$ A. Robson, ${ }^{56}$ E. Rocco, ${ }^{86}$ C. Roda, ${ }^{126 a, 126 b}$ Y. Rodina, ${ }^{88,00}$ S. Rodriguez Bosca, ${ }^{170}$ A. Rodriguez Perez, ${ }^{13}$ D. Rodriguez Rodriguez, ${ }^{170}$ S. Roe, ${ }^{32}$ C. S. Rogan, ${ }^{59}$ O. Røhne, ${ }^{121}$ J. Roloff,${ }^{59}$ A. Romaniouk, ${ }^{100}$ M. Romano, ${ }^{22 a, 22 b}$ S. M. Romano Saez, ${ }^{37}$ E. Romero Adam, ${ }^{170}$ N. Rompotis, ${ }^{77}$ M. Ronzani, ${ }^{51}$ L. Roos, ${ }^{83}$ S. Rosati, ${ }^{134 a}$ K. Rosbach, ${ }^{51}$ P. Rose, ${ }^{139}$ N.-A. Rosien, ${ }^{57}$ E. Rossi, ${ }^{106 a, 106 b}$ L. P. Rossi, ${ }^{53 \mathrm{a}}$ J. H. N. Rosten, ${ }^{30}$ R. Rosten, ${ }^{140}$ M. Rotaru, ${ }^{28 b}$ J. Rothberg, ${ }^{140}$ D. Rousseau, ${ }^{119}$ A. Rozanov, ${ }^{88}$ Y. Rozen, ${ }^{154}$ X. Ruan, ${ }^{147 \mathrm{c}}$ F. Rubbo, ${ }^{145}$ F. Rühr, ${ }^{51}$ A. Ruiz-Martinez, ${ }^{31}$ Z. Rurikova, ${ }^{51}$ N. A. Rusakovich, ${ }^{68}$ H. L. Russell, ${ }^{90}$

J. P. Rutherfoord, ${ }^{7}$ N. Ruthmann, ${ }^{32}$ Y. F. Ryabov, ${ }^{125}$ M. Rybar, ${ }^{169}$ G. Rybkin, ${ }^{119}$ S. Ryu, ${ }^{6}$ A. Ryzhov, ${ }^{132}$ G. F. Rzehorz,${ }^{57}$ A. F. Saavedra, ${ }^{152}$ G. Sabato, ${ }^{109}$ S. Sacerdoti, ${ }^{29}$ H. F-W. Sadrozinski, ${ }^{139}$ R. Sadykov, ${ }^{68}$ F. Safai Tehrani, ${ }^{134 a}$ P. Saha, ${ }^{110}$ M. Sahinsoy, ${ }^{60 a}$ M. Saimpert, ${ }^{45}$ M. Saito, ${ }^{157}$ T. Saito, ${ }^{157}$ H. Sakamoto, ${ }^{157}$ Y. Sakurai, ${ }^{174}$ G. Salamanna, ${ }^{136 a, 136 b}$

J. E. Salazar Loyola, ${ }^{34 \mathrm{~b}}$ D. Salek,${ }^{109}$ P. H. Sales De Bruin, ${ }^{168}$ D. Salihagic, ${ }^{103}$ A. Salnikov, ${ }^{145}$ J. Salt, ${ }^{170}$ D. Salvatore, ${ }^{40 a, 40 b}$ F. Salvatore, ${ }^{151}$ A. Salvucci, ${ }^{62 a, 62 b, 62 c}$ A. Salzburger, ${ }^{32}$ D. Sammel, ${ }^{51}$ D. Sampsonidis, ${ }^{156}$ D. Sampsonidou, ${ }^{156}$ J. Sánchez, ${ }^{170}$ V. Sanchez Martinez, ${ }^{170}$ A. Sanchez Pineda, ${ }^{167 a, 167 c}$ H. Sandaker, ${ }^{121}$ R. L. Sandbach ${ }^{79}$ C. O. Sander, ${ }^{45}$ M. Sandhoff, ${ }^{178}$ C. Sandoval, ${ }^{21}$ D. P. C. Sankey, ${ }^{133}$ M. Sannino,${ }^{53 a, 53 b}$ Y. Sano, ${ }^{105}$ A. Sansoni,${ }^{50}$ C. Santoni, ${ }^{37}$ H. Santos, ${ }^{128 a}$ I. Santoyo Castillo, ${ }^{151}$ A. Sapronov, ${ }^{68}$ J. G. Saraiva, ${ }^{128 a, 128 d}$ B. Sarrazin, ${ }^{23}$ O. Sasaki, ${ }^{69}$ K. Sato, ${ }^{164}$ E. Sauvan, ${ }^{5}$ G. Savage ${ }^{80}$

P. Savard, ${ }^{161, \mathrm{e}}$ N. Savic, ${ }^{103}$ C. Sawyer, ${ }^{133}$ L. Sawyer, ${ }^{82, \mathrm{v}}$ J. Saxon, ${ }^{33}$ C. Sbarra, ${ }^{2 \mathrm{a}}$ A. Sbrizzi, ${ }^{22 \mathrm{a}, 22 \mathrm{~b}}$ T. Scanlon, ${ }^{81}$ D. A. Scannicchio, ${ }^{166}$ M. Scarcella, ${ }^{152}$ J. Schaarschmidt, ${ }^{140}$ P. Schacht, ${ }^{103}$ B. M. Schachtner, ${ }^{102}$ D. Schaefer, ${ }^{32}$ L. Schaefer, ${ }^{124}$ R. Schaefer, ${ }^{45}$ J. Schaeffer, ${ }^{86}$ S. Schaepe ${ }^{23}$ S. Schaetzel, ${ }^{60 b}$ U. Schäfer, ${ }^{86}$ A. C. Schaffer, ${ }^{119}$ D. Schaile, ${ }^{102}$ R. D. Schamberger, ${ }^{150}$ V. A. Schegelsky, ${ }^{125}$ D. Scheirich, ${ }^{131}$ M. Schernau, ${ }^{166}$ C. Schiavi, ${ }^{53 a, 53 b}$ S. Schier, ${ }^{139}$ L. K. Schildgen, ${ }^{23}$ C. Schillo, ${ }^{51}$ M. Schioppa ${ }^{40 a, 40 b}$ S. Schlenker, ${ }^{32}$ K. R. Schmidt-Sommerfeld, ${ }^{103}$ K. Schmieden, ${ }^{32}$ C. Schmitt, ${ }^{86}$ S. Schmitt, ${ }^{45}$ S. Schmitz, ${ }^{86}$ U. Schnoor, ${ }^{51}$ L. Schoeffel, ${ }^{138}$ A. Schoening ${ }^{60 b}$ B. D. Schoenrock, ${ }^{93}$ E. Schopf,${ }^{23}$ M. Schott, ${ }^{86}$ J. F. P. Schouwenberg, ${ }^{108}$ J. Schovancova, ${ }^{32}$ S. Schramm, ${ }^{52}$ N. Schuh, ${ }^{86}$ A. Schulte, ${ }^{86}$ M. J. Schultens, ${ }^{23}$

H.-C. Schultz-Coulon, ${ }^{60 a}$ H. Schulz, ${ }^{17}$ M. Schumacher, ${ }^{51}$ B. A. Schumm, ${ }^{139}$ Ph. Schune, ${ }^{138}$ A. Schwartzman, ${ }^{145}$

T. A. Schwarz, ${ }^{92}$ H. Schweiger ${ }^{87}$ Ph. Schwemling, ${ }^{138}$ R. Schwienhorst, ${ }^{93}$ J. Schwindling, ${ }^{138}$ A. Sciandra, ${ }^{23}$ G. Sciolla, ${ }^{25}$ M. Scornajenghi, ${ }^{40 a, 40 b}$ F. Scuri, ${ }^{126 a, 126 b}$ F. Scutti, ${ }^{91}$ J. Searcy, ${ }^{92}$ P. Seema, ${ }^{23}$ S. C. Seidel,${ }^{107}$ A. Seiden, ${ }^{139}$ J. M. Seixas, ${ }^{26 a}$ G. Sekhniaidze, ${ }^{106 a}$ K. Sekhon, ${ }^{92}$ S. J. Sekula, ${ }^{43}$ N. Semprini-Cesari, ${ }^{22 a, 22 b}$ S. Senkin, ${ }^{37}$ C. Serfon, ${ }^{121}$ L. Serin, ${ }^{119}$

L. Serkin, ${ }^{167 a, 167 b}$ M. Sessa, ${ }^{136 a, 136 b}$ R. Seuster, ${ }^{172}$ H. Severini, ${ }^{115}$ T. Sfiligoj, ${ }^{78}$ F. Sforza, ${ }^{32}$ A. Sfyrla, ${ }^{52}$ E. Shabalina, ${ }^{57}$ N. W. Shaikh, ${ }^{148 a, 148 b}$ L. Y. Shan, ${ }^{35 a}$ R. Shang, ${ }^{169}$ J. T. Shank, ${ }^{24}$ M. Shapiro, ${ }^{16}$ P. B. Shatalov, ${ }^{99}$ K. Shaw, ${ }^{167 a, 167 b}$ S. M. Shaw,${ }^{87}$ A. Shcherbakova, ${ }^{148 a, 148 b}$ C. Y. Shehu, ${ }^{151}$ Y. Shen, ${ }^{115}$ N. Sherafati, ${ }^{31}$ P. Sherwood, ${ }^{81}$ L. Shi ${ }^{153, p p}$ S. Shimizu, ${ }^{70}$ C. O. Shimmin, ${ }^{179}$ M. Shimojima, ${ }^{104}$ I. P. J. Shipsey, ${ }^{122}$ S. Shirabe, ${ }^{73}$ M. Shiyakova, ${ }^{68, q 9}$ J. Shlomi, ${ }^{175}$ A. Shmeleva, ${ }^{98}$ D. Shoaleh Saadi, ${ }^{97}$ M. J. Shochet, ${ }^{33}$ S. Shojaii, ${ }^{94 a}$ D. R. Shope, ${ }^{115}$ S. Shrestha, ${ }^{113}$ E. Shulga, ${ }^{100}$ M. A. Shupe, ${ }^{7}$ P. Sicho, ${ }^{129}$ A. M. Sickles, ${ }^{169}$ P. E. Sidebo, ${ }^{149}$ E. Sideras Haddad ${ }^{147 \mathrm{c}}$ O. Sidiropoulou, ${ }^{177}$ A. Sidoti, ${ }^{22 a, 22 b}$ F. Siegert, ${ }^{47}$ Dj. Sijacki, ${ }^{14}$ J. Silva, ${ }^{128 a, 128 d}$ S. B. Silverstein, ${ }^{148 a}$ V. Simak, ${ }^{130}$ Lj. Simic, ${ }^{14}$ S. Simion, ${ }^{119}$ E. Simioni, ${ }^{86}$ B. Simmons, ${ }^{81}$ M. Simon, ${ }^{86}$ P. Sinervo, ${ }^{161}$ N. B. Sinev, ${ }^{118}$ M. Sioli, ${ }^{22 a, 22 b}$ G. Siragusa, ${ }^{177}$ I. Siral, ${ }^{92}$ S. Yu. Sivoklokov, ${ }^{101}$ J. Sjölin, ${ }^{148 a, 148 b}$ M. B. Skinner, ${ }^{75}$ P. Skubic, ${ }^{115}$ M. Slater, ${ }^{19}$ T. Slavicek, ${ }^{130}$ M. Slawinska, ${ }^{42}$ K. Sliwa, ${ }^{165}$ R. Slovak, ${ }^{131}$ V. Smakhtin, ${ }^{175}$ B. H. Smart, ${ }^{5}$ J. Smiesko, ${ }^{146 a}$ N. Smirnov, ${ }^{100}$ S. Yu. Smirnov, ${ }^{100}$ Y. Smirnov, ${ }^{100}$ L. N. Smirnova, ${ }^{101, r \mathrm{r}}$ O. Smirnova, ${ }^{84}$ J. W. Smith, ${ }^{57}$

M. N. K. Smith, ${ }^{38}$ R. W. Smith, ${ }^{38}$ M. Smizanska, ${ }^{75}$ K. Smolek, ${ }^{130}$ A. A. Snesarev, ${ }^{98}$ I. M. Snyder, ${ }^{118}$ S. Snyder, ${ }^{27}$ R. Sobie, ${ }^{172, p}$ F. Socher, ${ }^{47}$ A. Soffer, ${ }^{155}$ A. Søgaard,${ }^{49}$ D. A. Soh, ${ }^{153}$ G. Sokhrannyi, ${ }^{78}$ C. A. Solans Sanchez, ${ }^{32}$ M. Solar, ${ }^{130}$ E. Yu. Soldatov, ${ }^{100}$ U. Soldevila, ${ }^{170}$ A. A. Solodkov, ${ }^{132}$ A. Soloshenko, ${ }^{68}$ O. V. Solovyanov, ${ }^{132}$ V. Solovyev, ${ }^{125}$ P. Sommer, ${ }^{51}$ H. Son, ${ }^{165}$ A. Sopczak, ${ }^{130}$ D. Sosa, ${ }^{60 b}$ C. L. Sotiropoulou, ${ }^{126 a, 126 b}$ R. Soualah, ${ }^{167 a, 167 c}$ A. M. Soukharev, ${ }^{11, d}$ D. South, ${ }^{45}$

B. C. Sowden, ${ }^{80}$ S. Spagnolo, ${ }^{76 a, 76 b}$ M. Spalla, ${ }^{126 a, 126 b}$ M. Spangenberg, ${ }^{173}$ F. Spanò, ${ }^{80}$ D. Sperlich, ${ }^{17}$ F. Spettel,${ }^{103}$

T. M. Spieker, ${ }^{60 a}$ R. Spighi, ${ }^{22 a}$ G. Spigo, ${ }^{32}$ L. A. Spiller, ${ }^{91}$ M. Spousta, ${ }^{131}$ R. D. St. Denis, ${ }^{56, a}$ A. Stabile,${ }^{94 a}$ R. Stamen, ${ }^{60 a}$ S. Stamm, ${ }^{17}$ E. Stanecka, ${ }^{42}$ R. W. Stanek, ${ }^{6}$ C. Stanescu, ${ }^{136 a}$ M. M. Stanitzki, ${ }^{45}$ B. S. Stapf,${ }^{109}$ S. Stapnes, ${ }^{121}$

E. A. Starchenko, ${ }^{132}$ G. H. Stark, ${ }^{33}$ J. Stark ${ }^{58}$ S. H Stark, ${ }^{39}$ P. Staroba,${ }^{129}$ P. Starovoitov, ${ }^{60 a}$ S. Stärz, ${ }^{32}$ R. Staszewski, ${ }^{42}$ P. Steinberg, ${ }^{27}$ B. Stelzer, ${ }^{144}$ H. J. Stelzer, ${ }^{32}$ O. Stelzer-Chilton, ${ }^{163 a}$ H. Stenzel, ${ }^{55}$ G. A. Stewart,${ }^{56}$ M. C. Stockton, ${ }^{118}$ 
M. Stoebe ${ }^{90}$ G. Stoicea, ${ }^{28 b}$ P. Stolte, ${ }^{57}$ S. Stonjek, ${ }^{103}$ A. R. Stradling, ${ }^{8}$ A. Straessner, ${ }^{47}$ M. E. Stramaglia, ${ }^{18}$ J. Strandberg, ${ }^{149}$ S. Strandberg, ${ }^{148 a, 148 b}$ M. Strauss, ${ }^{115}$ P. Strizenec, ${ }^{146 b}$ R. Ströhmer, ${ }^{177}$ D. M. Strom, ${ }^{118}$ R. Stroynowski, ${ }^{43}$ A. Strubig, ${ }^{49}$

S. A. Stucci, ${ }^{27}$ B. Stugu, ${ }^{15}$ N. A. Styles, ${ }^{45}$ D. Su, ${ }^{145}$ J. Su, ${ }^{127}$ S. Suchek,${ }^{60 a}$ Y. Sugaya, ${ }^{120}$ M. Suk, ${ }^{130}$ V. V. Sulin, ${ }^{98}$

DMS Sultan, ${ }^{162 a, 162 b}$ S. Sultansoy, ${ }^{4 \mathrm{c}}$ T. Sumida, ${ }^{71}$ S. Sun, ${ }^{59}$ X. Sun, ${ }^{3}$ K. Suruliz, ${ }^{151}$ C. J. E. Suster, ${ }^{152}$ M. R. Sutton, ${ }^{151}$ S. Suzuki, ${ }^{69}$ M. Svatos, ${ }^{129}$ M. Swiatlowski, ${ }^{33}$ S. P. Swift, ${ }^{2}$ I. Sykora, ${ }^{146 a}$ T. Sykora, ${ }^{131}$ D. Ta, ${ }^{51}$ K. Tackmann,${ }^{45}$ J. Taenzer, ${ }^{155}$ A. Taffard, ${ }^{166}$ R. Tafirout, ${ }^{163 a}$ N. Taiblum, ${ }^{155}$ H. Takai, ${ }^{27}$ R. Takashima, ${ }^{72}$ E. H. Takasugi, ${ }^{103}$ T. Takeshita, ${ }^{142}$ Y. Takubo, ${ }^{69}$ M. Talby, ${ }^{88}$ A. A. Talyshev, ${ }^{11, d}$ J. Tanaka, ${ }^{157}$ M. Tanaka, ${ }^{159}$ R. Tanaka, ${ }^{119}$ S. Tanaka ${ }^{69}$ R. Tanioka, ${ }^{70}$ B. B. Tannenwald, ${ }^{113}$ S. Tapia Araya, ${ }^{34 \mathrm{~b}}$ S. Tapprogge ${ }^{86}$ S. Tarem, ${ }^{154}$ G. F. Tartarelli, ${ }^{94 a}$ P. Tas, ${ }^{131}$ M. Tasevsky, ${ }^{129}$ T. Tashiro, ${ }^{71}$ E. Tassi, ${ }^{40 a, 40 b}$

A. Tavares Delgado, ${ }^{128 a, 128 b}$ Y. Tayalati, ${ }^{137 \mathrm{e}}$ A. C. Taylor, ${ }^{107}$ G. N. Taylor, ${ }^{91}$ P. T. E. Taylor, ${ }^{91}$ W. Taylor, ${ }^{163 b}$

P. Teixeira-Dias,${ }^{80}$ D. Temple, ${ }^{144}$ H. Ten Kate,${ }^{32}$ P. K. Teng, ${ }^{153}$ J. J. Teoh,${ }^{120}$ F. Tepel, ${ }^{178}$ S. Terada, ${ }^{69}$ K. Terashi, ${ }^{157}$

J. Terron, ${ }^{85}$ S. Terzo, ${ }^{13}$ M. Testa, ${ }^{50}$ R. J. Teuscher ${ }^{161, p}$ T. Theveneaux-Pelzer, ${ }^{88}$ F. Thiele, ${ }^{39}$ J. P. Thomas, ${ }^{19}$

J. Thomas-Wilsker, ${ }^{80}$ P. D. Thompson, ${ }^{19}$ A. S. Thompson, ${ }^{56}$ L. A. Thomsen, ${ }^{179}$ E. Thomson, ${ }^{124}$ M. J. Tibbetts, ${ }^{16}$

R. E. Ticse Torres ${ }^{88}$ V. O. Tikhomirov, ${ }^{98, s s}$ Yu. A. Tikhonov, ${ }^{11, d}$ S. Timoshenko, ${ }^{100}$ P. Tipton, ${ }^{179}$ S. Tisserant ${ }^{88}$

K. Todome, ${ }^{159}$ S. Todorova-Nova, ${ }^{5}$ S. Todt, ${ }^{47}$ J. Tojo, ${ }^{73}$ S. Tokár, ${ }^{16 a}$ K. Tokushuku, ${ }^{69}$ E. Tolley, ${ }^{59}$ L. Tomlinson, ${ }^{87}$ M. Tomoto, ${ }^{105}$ L. Tompkins, ${ }^{145, \text { tt }}$ K. Toms,${ }^{107}$ B. Tong, ${ }^{59}$ P. Tornambe,${ }^{51}$ E. Torrence, ${ }^{118}$ H. Torres,${ }^{144}$ E. Torró Pastor, ${ }^{140}$ J. Toth,${ }^{88, \text { uu }}$ F. Touchard ${ }^{88}$ D. R. Tovey, ${ }^{141}$ C. J. Treado, ${ }^{112}$ T. Trefzger, ${ }^{177}$ F. Tresoldi, ${ }^{151}$ A. Tricoli, ${ }^{27}$ I. M. Trigger, ${ }^{163 a}$ S. Trincaz-Duvoid,${ }^{83}$ M. F. Tripiana,${ }^{13}$ W. Trischuk, ${ }^{161}$ B. Trocmé, ${ }^{58}$ A. Trofymov, ${ }^{45}$ C. Troncon, ${ }^{94 a}$ M. Trottier-McDonald, ${ }^{16}$ M. Trovatelli, ${ }^{172}$ L. Truong, ${ }^{147 b}$ M. Trzebinski, ${ }^{42}$ A. Trzupek, ${ }^{42}$ K. W. Tsang, ${ }^{62 \mathrm{a}}$ J. C-L. Tseng, ${ }^{122}$ P. V. Tsiareshka, ${ }^{95}$ G. Tsipolitis, ${ }^{10}$ N. Tsirintanis, ${ }^{9}$ S. Tsiskaridze, ${ }^{13}$ V. Tsiskaridze,${ }^{51}$ E. G. Tskhadadze,${ }^{54 a}$ K. M. Tsui, ${ }^{62 a}$ I. I. Tsukerman, ${ }^{99}$ V. Tsulaia, ${ }^{16}$ S. Tsuno, ${ }^{69}$ D. Tsybychev, ${ }^{150} \mathrm{Y}$. Tu, ${ }^{62 \mathrm{~b}}$ A. Tudorache, ${ }^{28 \mathrm{~b}}$ V. Tudorache, ${ }^{28 \mathrm{~b}}$ T. T. Tulbure, ${ }^{28 \mathrm{a}}$ A. N. Tuna, ${ }^{59}$ S. A. Tupputi, ${ }^{22 a, 22 b}$ S. Turchikhin, ${ }^{68}$ D. Turgeman, ${ }^{175}$ I. Turk Cakir, ${ }^{4 b, v v}$ R. Turra, ${ }^{94 a}$ P. M. Tuts, ${ }^{38}$ G. Ucchielli, ${ }^{22 a, 22 b}$ I. Ueda, ${ }^{69}$ M. Ughetto, ${ }^{148 a, 148 b}$ F. Ukegawa, ${ }^{164}$ G. Unal, ${ }^{32}$ A. Undrus, ${ }^{27}$ G. Unel, ${ }^{166}$ F. C. Ungaro, ${ }^{91}$ Y. Unno, ${ }^{69}$ C. Unverdorben, ${ }^{102}$ J. Urban, ${ }^{146 b}$ P. Urquijo, ${ }^{91}$ P. Urrejola, ${ }^{86}$ G. Usai, ${ }^{8}$ J. Usui, ${ }^{69}$ L. Vacavant, ${ }^{88}$ V. Vacek, ${ }^{130}$ B. Vachon, ${ }^{90}$ K. O. H. Vadla, ${ }^{121}$ A. Vaidya, ${ }^{81}$ C. Valderanis, ${ }^{102}$ E. Valdes Santurio, ${ }^{148 a, 148 b}$ S. Valentinetti, ${ }^{22 a, 22 b}$ A. Valero, ${ }^{170}$ L. Valéry, ${ }^{13}$ S. Valkar, ${ }^{131}$ A. Vallier, ${ }^{5}$ J. A. Valls Ferrer, ${ }^{170}$ W. Van Den Wollenberg, ${ }^{109}$ H. van der Graaf, ${ }^{109}$ P. van Gemmeren, ${ }^{6}$ J. Van Nieuwkoop, ${ }^{144}$ I. van Vulpen, ${ }^{109}$ M. C. van Woerden, ${ }^{109}$ M. Vanadia, ${ }^{135 a, 135 b}$ W. Vandelli, ${ }^{32}$ A. Vaniachine, ${ }^{160}$ P. Vankov, ${ }^{109}$ G. Vardanyan, ${ }^{180}$ R. Vari, ${ }^{134 a}$ E. W. Varnes, ${ }^{7}$ C. Varni, ${ }^{53 a, 53 b}$ T. Varol, ${ }^{43}$ D. Varouchas, ${ }^{119}$ A. Vartapetian, ${ }^{8}$ K. E. Varvell, ${ }^{152}$ J. G. Vasquez, ${ }^{179}$ G. A. Vasquez, ${ }^{34 b}$ F. Vazeille, ${ }^{37}$ T. Vazquez Schroeder ${ }^{90}$ J. Veatch,${ }^{57}$ V. Veeraraghavan, ${ }^{7}$ L. M. Veloce,${ }^{161}$ F. Veloso, ${ }^{128 a, 128 \mathrm{c}}$ S. Veneziano, ${ }^{134 \mathrm{a}}$ A. Ventura, ${ }^{76 \mathrm{a}, 76 \mathrm{~b}}$ M. Venturi, ${ }^{172}$ N. Venturi, ${ }^{32}$ A. Venturini, ${ }^{25}$ V. Vercesi, ${ }^{123 a}$ M. Verducci, ${ }^{136 a, 136 b}$ W. Verkerke, ${ }^{109}$ A. T. Vermeulen, ${ }^{109}$ J. C. Vermeulen, ${ }^{109}$ M. C. Vetterli, ${ }^{144, \mathrm{e}}$ N. Viaux Maira, ${ }^{34 \mathrm{~b}}$ O. Viazlo, ${ }^{84}$ I. Vichou, ${ }^{169, \mathrm{a}}$ T. Vickey, ${ }^{141}$ O. E. Vickey Boeriu, ${ }^{141}$ G. H. A. Viehhauser, ${ }^{122}$ S. Viel, ${ }^{16}$ L. Vigani, ${ }^{122}$ M. Villa, ${ }^{22 a, 22 b}$ M. Villaplana Perez,${ }^{94 a, 94 b}$ E. Vilucchi, ${ }^{50}$ M. G. Vincter, ${ }^{31}$ V. B. Vinogradov, ${ }^{68}$ A. Vishwakarma, ${ }^{45}$ C. Vittori, ${ }^{22 a, 22 b}$ I. Vivarelli, ${ }^{151}$ S. Vlachos, ${ }^{10}$ M. Vogel, ${ }^{178}$ P. Vokac,,${ }^{130}$ G. Volpi ${ }^{126 a, 126 b}$ H. von der Schmitt, ${ }^{103}$ E. von Toerne, ${ }^{23}$ V. Vorobel, ${ }^{131}$ K. Vorobev, ${ }^{100}$ M. Vos, ${ }^{170}$ R. Voss, ${ }^{32}$

J. H. Vossebeld, ${ }^{77}$ N. Vranjes, ${ }^{14}$ M. Vranjes Milosavljevic, ${ }^{14}$ V. Vrba, ${ }^{130}$ M. Vreeswijk, ${ }^{109}$ R. Vuillermet, ${ }^{32}$ I. Vukotic, ${ }^{33}$ P. Wagner, ${ }^{23}$ W. Wagner, ${ }^{178}$ J. Wagner-Kuhr, ${ }^{102}$ H. Wahlberg, ${ }^{74}$ S. Wahrmund ${ }^{47}$ J. Wakabayashi, ${ }^{105}$ J. Walder ${ }^{75}$ R. Walker, ${ }^{102}$ W. Walkowiak, ${ }^{143}$ V. Wallangen, ${ }^{148 a, 148 b}$ C. Wang, ${ }^{35 b}$ C. Wang,${ }^{36 b, w w}$ F. Wang, ${ }^{176}$ H. Wang, ${ }^{16}$ H. Wang, ${ }^{3}$ J. Wang, ${ }^{45}$ J. Wang, ${ }^{152}$ Q. Wang, ${ }^{115}$ R.-J. Wang, ${ }^{83}$ R. Wang, ${ }^{6}$ S. M. Wang, ${ }^{153}$ T. Wang, ${ }^{38}$ W. Wang, ${ }^{153, x x}$ W. Wang, ${ }^{36 a}$ Z. Wang, ${ }^{36 c}$ C. Wanotayaroj, ${ }^{118}$ A. Warburton, ${ }^{90}$ C. P. Ward, ${ }^{30}$ D. R. Wardrope, ${ }^{81}$ A. Washbrook, ${ }^{49}$ P. M. Watkins, ${ }^{19}$ A. T. Watson, ${ }^{19}$ M. F. Watson, ${ }^{19}$ G. Watts, ${ }^{140}$ S. Watts,${ }^{87}$ B. M. Waugh,${ }^{81}$ A. F. Webb, ${ }^{11}$ S. Webb, ${ }^{86}$ M. S. Weber, ${ }^{18}$ S. W. Weber, ${ }^{177}$ S. A. Weber, ${ }^{31}$ J. S. Webster, ${ }^{6}$ A. R. Weidberg, ${ }^{122}$ B. Weinert, ${ }^{64}$ J. Weingarten, ${ }^{57}$ M. Weirich,${ }^{86}$ C. Weiser, ${ }^{51}$ H. Weits, ${ }^{109}$ P. S. Wells, ${ }^{32}$ T. Wenaus, ${ }^{27}$ T. Wengler, ${ }^{32}$ S. Wenig, ${ }^{32}$ N. Wermes, ${ }^{23}$ M. D. Werner, ${ }^{67}$ P. Werner, ${ }^{32}$ M. Wessels, ${ }^{60 a}$ K. Whalen, ${ }^{118}$ N. L. Whallon, ${ }^{140}$ A. M. Wharton, ${ }^{75}$ A. S. White, ${ }^{92}$ A. White, ${ }^{8}$ M. J. White, ${ }^{1}$ R. White, ${ }^{34 b}$ D. Whiteson, ${ }^{166}$ B. W. Whitmore, ${ }^{75}$ F. J. Wickens, ${ }^{133}$ W. Wiedenmann, ${ }^{176}$ M. Wielers, ${ }^{133}$ C. Wiglesworth, ${ }^{39}$ L. A. M. Wiik-Fuchs, ${ }^{51}$ A. Wildauer, ${ }^{103}$ F. Wilk, ${ }^{87}$ H. G. Wilkens, ${ }^{32}$ H. H. Williams, ${ }^{124}$ S. Williams, ${ }^{109}$ C. Willis, ${ }^{93}$ S. Willocq, ${ }^{89}$ J. A. Wilson, ${ }^{19}$ I. Wingerter-Seez, ${ }^{5}$ E. Winkels, ${ }^{151}$ F. Winklmeier, ${ }^{118}$ O. J. Winston, ${ }^{151}$ B. T. Winter ${ }^{23}$ M. Wittgen, ${ }^{145}$ M. Wobisch, ${ }^{82, v}$ T. M. H. Wolf, ${ }^{109}$ R. Wolff, ${ }^{88}$ M. W. Wolter, ${ }^{42}$ H. Wolters, ${ }^{128 a, 128 c}$ V. W. S. Wong, ${ }^{171}$ S. D. Worm, ${ }^{19}$ B. K. Wosiek, ${ }^{42}$ J. Wotschack, ${ }^{32}$ K. W. Wozniak, ${ }^{42}$ M. Wu, ${ }^{33}$ S. L. Wu, ${ }^{176}$ X. Wu, ${ }^{52}$ Y. Wu, ${ }^{92}$ T. R. Wyatt, ${ }^{87}$ B. M. Wynne, ${ }^{49}$ S. Xella, ${ }^{39}$ Z. Xi, ${ }^{92}$ L. Xia, ${ }^{35 c}$ D. Xu, ${ }^{35 a}$ L. Xu, ${ }^{27}$ T. Xu, ${ }^{138}$ B. Yabsley, ${ }^{152}$ S. Yacoob,${ }^{147 a}$ D. Yamaguchi, ${ }^{159}$ Y. Yamaguchi, ${ }^{120}$ 
A. Yamamoto ${ }^{69}$ S. Yamamoto, ${ }^{157}$ T. Yamanaka, ${ }^{157}$ M. Yamatani, ${ }^{157}$ K. Yamauchi, ${ }^{105}$ Y. Yamazaki, ${ }^{70}$ Z. Yan, ${ }^{24}$ H. Yang, ${ }^{36 c}$ H. Yang, ${ }^{16}$ Y. Yang, ${ }^{153}$ Z. Yang, ${ }^{15}$ W-M. Yao, ${ }^{16}$ Y. C. Yap ${ }^{83}$ Y. Yasu, ${ }^{69}$ E. Yatsenko, ${ }^{5}$ K. H. Yau Wong, ${ }^{23}$ J. Ye, ${ }^{43}$ S. Ye, ${ }^{27}$ I. Yeletskikh, ${ }^{68}$ E. Yigitbasi, ${ }^{24}$ E. Yildirim, ${ }^{86}$ K. Yorita, ${ }^{174}$ K. Yoshihara, ${ }^{124}$ C. Young, ${ }^{145}$ C. J. S. Young, ${ }^{32}$ J. Yu, ${ }^{8}$ J. Yu,${ }^{67}$ S. P. Y. Yuen, ${ }^{23}$ I. Yusuff, ${ }^{30, y y}$ B. Zabinski, ${ }^{42}$ G. Zacharis, ${ }^{10}$ R. Zaidan, ${ }^{13}$ A. M. Zaitsev, ${ }^{132, m m}$ N. Zakharchuk, ${ }^{45}$ J. Zalieckas, ${ }^{15}$ A. Zaman, ${ }^{150}$ S. Zambito, ${ }^{59}$ D. Zanzi, ${ }^{91}$ C. Zeitnitz, ${ }^{178}$ G. Zemaityte, ${ }^{122}$ A. Zemla, ${ }^{41 a}$ J. C. Zeng, ${ }^{169}$ Q. Zeng, ${ }^{145}$ O. Zenin, ${ }^{132}$ T. Ženiš, ${ }^{146 a}$ D. Zerwas, ${ }^{119}$ D. Zhang, ${ }^{92}$ F. Zhang, ${ }^{176}$ G. Zhang, ${ }^{36 a, z z}$ H. Zhang, ${ }^{35 b}$ J. Zhang, ${ }^{6}$ L. Zhang, ${ }^{51}$ L. Zhang, ${ }^{36 a}$ M. Zhang, ${ }^{169}$ P. Zhang, ${ }^{35 b}$ R. Zhang, ${ }^{23}$ R. Zhang, ${ }^{36 a, w w}$ X. Zhang, ${ }^{36 b}$ Y. Zhang, ${ }^{35 a}$ Z. Zhang, ${ }^{119}$ X. Zhao, ${ }^{43}$ Y. Zhao, ${ }^{36 b, a a a}$ Z. Zhao, ${ }^{36 a}$ A. Zhemchugov, ${ }^{68}$ B. Zhou, ${ }^{92}$ C. Zhou, ${ }^{176}$ L. Zhou, ${ }^{43}$ M. Zhou, ${ }^{35 a}$ M. Zhou, ${ }^{150}$ N. Zhou, ${ }^{35 \mathrm{c}}$ C. G. Zhu, ${ }^{36 \mathrm{~b}}$ H. Zhu, ${ }^{35 \mathrm{a}}$ J. Zhu, ${ }^{92}$ Y. Zhu, ${ }^{36 \mathrm{a}}$ X. Zhuang, ${ }^{35 \mathrm{a}}$ K. Zhukov, ${ }^{98}$ A. Zibell, ${ }^{177}$ D. Zieminska, ${ }^{64}$ N. I. Zimine, ${ }^{68}$ C. Zimmermann, ${ }^{86}$ S. Zimmermann, ${ }^{51}$ Z. Zinonos, ${ }^{103}$ M. Zinser, ${ }^{86}$ M. Ziolkowski, ${ }^{143}$ L. Živković, ${ }^{14}$ G. Zobernig, ${ }^{176}$ A. Zoccoli, ${ }^{22 a, 22 b}$ R. Zou, ${ }^{33}$ M. zur Nedden, ${ }^{17}$ and L. Zwalinski ${ }^{32}$

(ATLAS Collaboration)

${ }^{1}$ Department of Physics, University of Adelaide, Adelaide, Australia

${ }^{2}$ Physics Department, SUNY Albany, Albany New York, USA

${ }^{3}$ Department of Physics, University of Alberta, Edmonton Alberta, Canada

${ }^{4 a}$ Department of Physics, Ankara University, Ankara, Turkey

${ }^{4 \mathrm{~b}}$ Istanbul Aydin University, Istanbul, Turkey

${ }^{4 \mathrm{c}}$ Division of Physics, TOBB University of Economics and Technology, Ankara, Turkey

${ }^{5}$ LAPP, CNRS/IN2P3 and Université Savoie Mont Blanc, Annecy-le-Vieux, France

${ }^{6}$ High Energy Physics Division, Argonne National Laboratory, Argonne Illinois, USA

${ }^{7}$ Department of Physics, University of Arizona, Tucson Arizona, USA

${ }^{8}$ Department of Physics, The University of Texas at Arlington, Arlington Texas, USA

${ }^{9}$ Physics Department, National and Kapodistrian University of Athens, Athens, Greece

${ }^{10}$ Physics Department, National Technical University of Athens, Zografou, Greece

${ }^{11}$ Department of Physics, The University of Texas at Austin, Austin Texas, USA

${ }^{12}$ Institute of Physics, Azerbaijan Academy of Sciences, Baku, Azerbaijan

${ }^{13}$ Institut de Física d'Altes Energies (IFAE), The Barcelona Institute of Science and Technology, Barcelona, Spain

${ }^{14}$ Institute of Physics, University of Belgrade, Belgrade, Serbia

${ }^{15}$ Department for Physics and Technology, University of Bergen, Bergen, Norway

${ }^{16}$ Physics Division, Lawrence Berkeley National Laboratory and University of California, Berkeley California, USA

${ }^{17}$ Department of Physics, Humboldt University, Berlin, Germany

${ }^{18}$ Albert Einstein Center for Fundamental Physics and Laboratory for High Energy Physics, University of Bern, Bern, Switzerland

${ }^{19}$ School of Physics and Astronomy, University of Birmingham, Birmingham, United Kingdom

${ }^{20 a}$ Department of Physics, Bogazici University, Istanbul, Turkey

${ }^{20 \mathrm{~b}}$ Department of Physics Engineering, Gaziantep University, Gaziantep, Turkey

${ }^{20 \mathrm{c}}$ Istanbul Bilgi University, Faculty of Engineering and Natural Sciences, Istanbul, Turkey

${ }^{20 \mathrm{~d} B a h c e s e h i r}$ University, Faculty of Engineering and Natural Sciences, Istanbul, Turkey

${ }^{21}$ Centro de Investigaciones, Universidad Antonio Narino, Bogota, Colombia

${ }^{22 \mathrm{a}}$ INFN Sezione di Bologna, Bologna, Italy

${ }^{22 \mathrm{~b}}$ Dipartimento di Fisica e Astronomia, Università di Bologna, Bologna, Italy

${ }^{23}$ Physikalisches Institut, University of Bonn, Bonn, Germany

${ }^{24}$ Department of Physics, Boston University, Boston Massachusetts, USA

${ }^{25}$ Department of Physics, Brandeis University, Waltham Massachusetts, USA

${ }^{26 a}$ Universidade Federal do Rio De Janeiro COPPE/EE/IF, Rio de Janeiro, Brazil

${ }^{26 \mathrm{~b}}$ Electrical Circuits Department, Federal University of Juiz de Fora (UFJF), Juiz de Fora, Brazil

${ }^{26 c}$ Federal University of Sao Joao del Rei (UFSJ), Sao Joao del Rei, Brazil

${ }^{26 \mathrm{~d}}$ Instituto de Fisica, Universidade de Sao Paulo, Sao Paulo, Brazil

${ }^{27}$ Physics Department, Brookhaven National Laboratory, Upton New York, USA

${ }^{28 a}$ Transilvania University of Brasov, Brasov, Romania

${ }^{28 \mathrm{~b}}$ Horia Hulubei National Institute of Physics and Nuclear Engineering, Bucharest, Romania

${ }^{28 \mathrm{c}}$ Department of Physics, Alexandru Ioan Cuza University of Iasi, Iasi, Romania 
${ }^{28 \mathrm{~d}}$ National Institute for Research and Development of Isotopic and Molecular Technologies, Physics Department, Cluj Napoca, Romania

${ }^{28 \mathrm{e}}$ University Politehnica Bucharest, Bucharest, Romania

${ }^{28 \mathrm{f}}$ West University in Timisoara, Timisoara, Romania

${ }^{29}$ Departamento de Física, Universidad de Buenos Aires, Buenos Aires, Argentina

${ }^{30}$ Cavendish Laboratory, University of Cambridge, Cambridge, United Kingdom

${ }^{31}$ Department of Physics, Carleton University, Ottawa Ontario, Canada

${ }^{32}$ CERN, Geneva, Switzerland

${ }^{33}$ Enrico Fermi Institute, University of Chicago, Chicago Illinois, USA

${ }^{34 a}$ Departamento de Física, Pontificia Universidad Católica de Chile, Santiago, Chile

${ }^{34 \mathrm{~b}}$ Departamento de Física, Universidad Técnica Federico Santa María, Valparaíso, Chile

${ }^{35 a}$ Institute of High Energy Physics, Chinese Academy of Sciences, Beijing, China

${ }^{35 \mathrm{~b}}$ Department of Physics, Nanjing University, Jiangsu, China

${ }^{35 c}$ Physics Department, Tsinghua University, Beijing, China

${ }^{36 a}$ Department of Modern Physics and State Key Laboratory of Particle Detection and Electronics, University of Science and Technology of China, Anhui, China

${ }^{36 \mathrm{~b}}$ School of Physics, Shandong University, Shandong, China

${ }^{36 c}$ Department of Physics and Astronomy, Key Laboratory for Particle Physics, Astrophysics and Cosmology, Ministry of Education; Shanghai Key Laboratory for Particle Physics and Cosmology,

Shanghai Jiao Tong University, Shanghai(also at PKU-CHEP), China

${ }^{37}$ Université Clermont Auvergne, CNRS/IN2P3, LPC, Clermont-Ferrand, France

${ }^{38}$ Nevis Laboratory, Columbia University, Irvington New York, USA

${ }^{39}$ Niels Bohr Institute, University of Copenhagen, Kobenhavn, Denmark

${ }^{40 a}$ INFN Gruppo Collegato di Cosenza, Laboratori Nazionali di Frascati, Italy

${ }^{40 \mathrm{~b}}$ Dipartimento di Fisica, Università della Calabria, Rende, Italy

${ }^{41 \mathrm{a}}$ AGH University of Science and Technology, Faculty of Physics and Applied Computer Science, Krakow, Poland

${ }^{41 \mathrm{~b}}$ Marian Smoluchowski Institute of Physics, Jagiellonian University, Krakow, Poland

${ }^{42}$ Institute of Nuclear Physics Polish Academy of Sciences, Krakow, Poland

${ }^{43}$ Physics Department, Southern Methodist University, Dallas Texas, USA

${ }^{44}$ Physics Department, University of Texas at Dallas, Richardson Texas, USA

${ }^{45}$ DESY, Hamburg and Zeuthen, Germany

${ }^{46}$ Lehrstuhl für Experimentelle Physik IV, Technische Universität Dortmund, Dortmund, Germany

${ }^{47}$ Institut für Kern- und Teilchenphysik, Technische Universität Dresden, Dresden, Germany

${ }^{48}$ Department of Physics, Duke University, Durham North Carolina, USA

${ }^{49}$ SUPA - School of Physics and Astronomy, University of Edinburgh, Edinburgh, United Kingdom

${ }^{50}$ INFN e Laboratori Nazionali di Frascati, Frascati, Italy

${ }^{51}$ Fakultät für Mathematik und Physik, Albert-Ludwigs-Universität, Freiburg, Germany

${ }^{52}$ Departement de Physique Nucleaire et Corpusculaire, Université de Genève, Geneva, Switzerland

${ }^{53 a}$ INFN Sezione di Genova, Italy

${ }^{53 \mathrm{~b}}$ Dipartimento di Fisica, Università di Genova, Genova, Italy

${ }^{54 a}$ E. Andronikashvili Institute of Physics, Iv. Javakhishvili Tbilisi State University, Tbilisi, Georgia

${ }^{54 \mathrm{~b}}$ High Energy Physics Institute, Tbilisi State University, Tbilisi, Georgia

${ }^{55}$ II Physikalisches Institut, Justus-Liebig-Universität Giessen, Giessen, Germany

${ }^{56}$ SUPA - School of Physics and Astronomy, University of Glasgow, Glasgow, United Kingdom

${ }^{57}$ II Physikalisches Institut, Georg-August-Universität, Göttingen, Germany

${ }^{58}$ Laboratoire de Physique Subatomique et de Cosmologie, Université Grenoble-Alpes, CNRS/IN2P3, Grenoble, France

${ }^{59}$ Laboratory for Particle Physics and Cosmology, Harvard University, Cambridge Massachusetts, USA

${ }^{60 \mathrm{a}}$ Kirchhoff-Institut für Physik, Ruprecht-Karls-Universität Heidelberg, Heidelberg, Germany

${ }^{60 \mathrm{~b}}$ Physikalisches Institut, Ruprecht-Karls-Universität Heidelberg, Heidelberg, Germany

${ }^{61}$ Faculty of Applied Information Science, Hiroshima Institute of Technology, Hiroshima, Japan

${ }^{62 a}$ Department of Physics, The Chinese University of Hong Kong, Shatin, N.T., Hong Kong, China

${ }^{62 \mathrm{~b}}$ Department of Physics, The University of Hong Kong, Hong Kong, China

${ }^{62 c}$ Department of Physics and Institute for Advanced Study, The Hong Kong University of Science and

Technology, Clear Water Bay, Kowloon, Hong Kong, China

${ }^{63}$ Department of Physics, National Tsing Hua University, Hsinchu, Taiwan

${ }^{64}$ Department of Physics, Indiana University, Bloomington Indiana, USA

${ }^{65}$ Institut für Astro- und Teilchenphysik, Leopold-Franzens-Universität, Innsbruck, Austria

${ }^{66}$ University of Iowa, Iowa City Iowa, USA 
${ }^{67}$ Department of Physics and Astronomy, Iowa State University, Ames Iowa, USA

${ }^{68}$ Joint Institute for Nuclear Research, JINR Dubna, Dubna, Russia

${ }^{69}$ KEK, High Energy Accelerator Research Organization, Tsukuba, Japan

${ }^{70}$ Graduate School of Science, Kobe University, Kobe, Japan

${ }^{71}$ Faculty of Science, Kyoto University, Kyoto, Japan

${ }^{72}$ Kyoto University of Education, Kyoto, Japan

${ }^{73}$ Research Center for Advanced Particle Physics and Department of Physics, Kyushu University, Fukuoka, Japan

${ }^{74}$ Instituto de Física La Plata, Universidad Nacional de La Plata and CONICET, La Plata, Argentina

${ }^{75}$ Physics Department, Lancaster University, Lancaster, United Kingdom

${ }^{76 a}$ INFN Sezione di Lecce, Lecce, Italy

${ }^{76 \mathrm{~b}}$ Dipartimento di Matematica e Fisica, Università del Salento, Lecce, Italy

${ }^{77}$ Oliver Lodge Laboratory, University of Liverpool, Liverpool, United Kingdom

${ }^{78}$ Department of Experimental Particle Physics, Jožef Stefan Institute and Department of Physics, University of Ljubljana, Ljubljana, Slovenia

${ }^{79}$ School of Physics and Astronomy, Queen Mary University of London, London, United Kingdom

${ }^{80}$ Department of Physics, Royal Holloway University of London, Surrey, United Kingdom

${ }^{81}$ Department of Physics and Astronomy, University College London, London, United Kingdom

${ }^{82}$ Louisiana Tech University, Ruston Louisiana, USA

${ }^{83}$ Laboratoire de Physique Nucléaire et de Hautes Energies, UPMC and Université Paris-Diderot and CNRS/IN2P3, Paris, France

${ }^{84}$ Fysiska institutionen, Lunds universitet, Lund, Sweden

${ }^{85}$ Departamento de Fisica Teorica C-15, Universidad Autonoma de Madrid, Madrid, Spain

${ }^{86}$ Institut für Physik, Universität Mainz, Mainz, Germany

${ }^{87}$ School of Physics and Astronomy, University of Manchester, Manchester, United Kingdom

${ }^{88}$ CPPM, Aix-Marseille Université and CNRS/IN2P3, Marseille, France

${ }^{89}$ Department of Physics, University of Massachusetts, Amherst Massachusetts, USA

${ }^{90}$ Department of Physics, McGill University, Montreal Quebec, Canada

${ }^{91}$ School of Physics, University of Melbourne, Victoria, Australia

${ }^{92}$ Department of Physics, The University of Michigan, Ann Arbor Michigan, USA

${ }^{93}$ Department of Physics and Astronomy, Michigan State University, East Lansing Michigan, USA

${ }^{94 a}$ INFN Sezione di Milano, Milano, Italy

${ }^{94 \mathrm{~b}}$ Dipartimento di Fisica, Università di Milano, Milano, Italy

${ }^{95}$ B.I. Stepanov Institute of Physics, National Academy of Sciences of Belarus, Minsk, Republic of Belarus

${ }^{96}$ Research Institute for Nuclear Problems of Byelorussian State University, Minsk, Republic of Belarus

${ }^{97}$ Group of Particle Physics, University of Montreal, Montreal Quebec, Canada

${ }^{98}$ P.N. Lebedev Physical Institute of the Russian Academy of Sciences, Moscow, Russia

${ }^{99}$ Institute for Theoretical and Experimental Physics (ITEP), Moscow, Russia

${ }^{100}$ National Research Nuclear University MEPhI, Moscow, Russia

${ }^{101}$ D.V. Skobeltsyn Institute of Nuclear Physics, M.V. Lomonosov Moscow State University, Moscow, Russia

${ }^{102}$ Fakultät für Physik, Ludwig-Maximilians-Universität München, München, Germany

${ }^{103}$ Max-Planck-Institut für Physik (Werner-Heisenberg-Institut), München, Germany

${ }^{104}$ Nagasaki Institute of Applied Science, Nagasaki, Japan

${ }^{105}$ Graduate School of Science and Kobayashi-Maskawa Institute, Nagoya University, Nagoya, Japan

${ }^{106 a}$ INFN Sezione di Napoli, Italy

${ }^{106 \mathrm{~b}}$ Dipartimento di Fisica, Università di Napoli, Napoli, Italy

${ }^{107}$ Department of Physics and Astronomy, University of New Mexico, Albuquerque New Mexico, USA

${ }^{108}$ Institute for Mathematics, Astrophysics and Particle Physics, Radboud University Nijmegen/Nikhef, Nijmegen, Netherlands

${ }^{109}$ Nikhef National Institute for Subatomic Physics and University of Amsterdam, Amsterdam, Netherlands

${ }^{110}$ Department of Physics, Northern Illinois University, DeKalb Illinois, USA

${ }^{111}$ Budker Institute of Nuclear Physics, SB RAS, Novosibirsk, Russia

${ }^{112}$ Department of Physics, New York University, New York, New York, USA

${ }^{113}$ Ohio State University, Columbus Ohio, USA

${ }^{114}$ Faculty of Science, Okayama University, Okayama, Japan

${ }^{115}$ Homer L. Dodge Department of Physics and Astronomy, University of Oklahoma, Norman Oklahoma, USA

${ }^{116}$ Department of Physics, Oklahoma State University, Stillwater Oklahoma, USA

${ }^{117}$ Palacký University, RCPTM, Olomouc, Czech Republic 
${ }^{118}$ Center for High Energy Physics, University of Oregon, Eugene Oregon, USA

${ }^{119}$ LAL, Univ. Paris-Sud, CNRS/IN2P3, Université Paris-Saclay, Orsay, France

${ }^{120}$ Graduate School of Science, Osaka University, Osaka, Japan

${ }^{121}$ Department of Physics, University of Oslo, Oslo, Norway

${ }^{122}$ Department of Physics, Oxford University, Oxford, United Kingdom

${ }^{123 a}$ INFN Sezione di Pavia, Pavia, Italy

${ }^{123 \mathrm{~b}}$ Dipartimento di Fisica, Università di Pavia, Pavia, Italy

${ }^{124}$ Department of Physics, University of Pennsylvania, Philadelphia Pennsylvania, USA

${ }^{125}$ National Research Centre "Kurchatov Institute” B.P.Konstantinov Petersburg Nuclear Physics Institute, St. Petersburg, Russia

${ }^{126 a}$ INFN Sezione di Pisa, Pisa, Italy

${ }^{126 \mathrm{~b}}$ Dipartimento di Fisica E. Fermi, Università di Pisa, Pisa, Italy

${ }^{127}$ Department of Physics and Astronomy, University of Pittsburgh, Pittsburgh Pennsylvania, USA

${ }^{128 a}$ Laboratório de Instrumentação e Física Experimental de Partículas - LIP, Lisboa, Portugal

${ }^{128 \mathrm{~b}}$ Faculdade de Ciências, Universidade de Lisboa, Lisboa, Portugal

${ }^{128 c}$ Department of Physics, University of Coimbra, Coimbra, Portugal

${ }^{128 \mathrm{~d}}$ Centro de Física Nuclear da Universidade de Lisboa, Lisboa, Portugal

${ }^{128 \mathrm{e}}$ Departamento de Fisica, Universidade do Minho, Braga, Portugal

${ }^{128 \mathrm{f}}$ Departamento de Fisica Teorica y del Cosmos and CAFPE, Universidad de Granada, Granada, Portugal

${ }^{128 g}$ Dep Fisica and CEFITEC of Faculdade de Ciencias e Tecnologia, Universidade Nova de Lisboa, Caparica, Portugal

${ }^{129}$ Institute of Physics, Academy of Sciences of the Czech Republic, Praha, Czech Republic

${ }^{130}$ Czech Technical University in Prague, Praha, Czech Republic

${ }^{131}$ Charles University, Faculty of Mathematics and Physics, Prague, Czech Republic

${ }^{132}$ State Research Center Institute for High Energy Physics (Protvino), NRC KI, Russia

${ }^{133}$ Particle Physics Department, Rutherford Appleton Laboratory, Didcot, United Kingdom

${ }^{134 a}$ INFN Sezione di Roma, Roma, Italy

${ }^{134 b}$ Dipartimento di Fisica, Sapienza Università di Roma, Roma, Italy

${ }^{135 a}$ INFN Sezione di Roma Tor Vergata, Roma, Italy

${ }^{135 \mathrm{~b}}$ Dipartimento di Fisica, Università di Roma Tor Vergata, Roma, Italy

${ }^{136 a}$ INFN Sezione di Roma Tre, Roma, Italy

${ }^{136 \mathrm{~b}}$ Dipartimento di Matematica e Fisica, Università Roma Tre, Roma, Italy

${ }^{137 a}$ Faculté des Sciences Ain Chock, Réseau Universitaire de Physique des Hautes Energies - Université

Hassan II, Casablanca, Morocco

${ }^{137 \mathrm{~b}}$ Centre National de l'Energie des Sciences Techniques Nucleaires, Rabat, Morocco

${ }^{137 c}$ Faculté des Sciences Semlalia, Université Cadi Ayyad, LPHEA-Marrakech, Morocco

${ }^{137 \mathrm{~d}}$ Faculté des Sciences, Université Mohamed Premier and LPTPM, Oujda, Morocco

${ }^{137 \mathrm{e}}$ Faculté des sciences, Université Mohammed V, Rabat, Morocco

${ }^{138}$ DSM/IRFU (Institut de Recherches sur les Lois Fondamentales de l'Univers),

CEA Saclay (Commissariat à l'Energie Atomique et aux Energies Alternatives), Gif-sur-Yvette, France

${ }^{139}$ Santa Cruz Institute for Particle Physics, University of California Santa Cruz, Santa Cruz California, USA

${ }^{140}$ Department of Physics, University of Washington, Seattle Washington, USA

${ }^{141}$ Department of Physics and Astronomy, University of Sheffield, Sheffield, United Kingdom

${ }^{142}$ Department of Physics, Shinshu University, Nagano, Japan

${ }^{143}$ Department Physik, Universität Siegen, Siegen, Germany

${ }^{144}$ Department of Physics, Simon Fraser University, Burnaby British Columbia, Canada

${ }^{145}$ SLAC National Accelerator Laboratory, Stanford California, USA

${ }^{146 a}$ Faculty of Mathematics, Physics \& Informatics, Comenius University, Bratislava, Slovak Republic

${ }^{146 \mathrm{~b}}$ Department of Subnuclear Physics, Institute of Experimental Physics of the Slovak Academy of

Sciences, Kosice, Slovak Republic

${ }^{147 a}$ Department of Physics, University of Cape Town, Cape Town, South Africa

${ }^{147 \mathrm{~b}}$ Department of Physics, University of Johannesburg, Johannesburg, South Africa

${ }^{147 \mathrm{c}}$ School of Physics, University of the Witwatersrand, Johannesburg, South Africa

${ }^{148 a}$ Department of Physics, Stockholm University, Sweden

${ }^{148 \mathrm{~b}}$ The Oskar Klein Centre, Stockholm, Sweden

${ }^{149}$ Physics Department, Royal Institute of Technology, Stockholm, Sweden 
${ }^{150}$ Departments of Physics \& Astronomy and Chemistry, Stony Brook University, Stony Brook New York, USA

${ }^{151}$ Department of Physics and Astronomy, University of Sussex, Brighton, United Kingdom

${ }^{152}$ School of Physics, University of Sydney, Sydney, Australia

${ }^{153}$ Institute of Physics, Academia Sinica, Taipei, Taiwan

${ }^{154}$ Department of Physics, Technion: Israel Institute of Technology, Haifa, Israel

${ }^{155}$ Raymond and Beverly Sackler School of Physics and Astronomy, Tel Aviv University, Tel Aviv, Israel

${ }^{156}$ Department of Physics, Aristotle University of Thessaloniki, Thessaloniki, Greece

${ }^{157}$ International Center for Elementary Particle Physics and Department of Physics, The University of Tokyo, Tokyo, Japan

${ }^{158}$ Graduate School of Science and Technology, Tokyo Metropolitan University, Tokyo, Japan

${ }^{159}$ Department of Physics, Tokyo Institute of Technology, Tokyo, Japan

${ }^{160}$ Tomsk State University, Tomsk, Russia

${ }^{161}$ Department of Physics, University of Toronto, Toronto Ontario, Canada

${ }^{162 a}$ INFN-TIFPA, Trento, Italy

${ }^{162 \mathrm{~b}}$ University of Trento, Trento, Italy

${ }^{163 a}$ TRIUMF, Vancouver British Columbia, Canada

${ }^{163 \mathrm{~b}}$ Department of Physics and Astronomy, York University, Toronto Ontario, Canada

${ }^{164}$ Faculty of Pure and Applied Sciences, and Center for Integrated Research in Fundamental Science and Engineering, University of Tsukuba, Tsukuba, Japan

${ }^{165}$ Department of Physics and Astronomy, Tufts University, Medford Massachusetts, USA

${ }^{166}$ Department of Physics and Astronomy, University of California Irvine, Irvine California, USA

${ }^{167 a}$ INFN Gruppo Collegato di Udine, Sezione di Trieste, Udine, Italy

${ }^{167 \mathrm{~b}}$ ICTP, Trieste, Italy

${ }^{167 \mathrm{c}}$ Dipartimento di Chimica, Fisica e Ambiente, Università di Udine, Udine, Italy

${ }^{168}$ Department of Physics and Astronomy, University of Uppsala, Uppsala, Sweden

${ }^{169}$ Department of Physics, University of Illinois, Urbana Illinois, USA

${ }^{170}$ Instituto de Fisica Corpuscular (IFIC), Centro Mixto Universidad de Valencia - CSIC, Spain

${ }^{171}$ Department of Physics, University of British Columbia, Vancouver British Columbia, Canada

${ }^{172}$ Department of Physics and Astronomy, University of Victoria, Victoria British Columbia, Canada

${ }^{173}$ Department of Physics, University of Warwick, Coventry, United Kingdom

${ }^{174}$ Waseda University, Tokyo, Japan

${ }^{175}$ Department of Particle Physics, The Weizmann Institute of Science, Rehovot, Israel

${ }^{176}$ Department of Physics, University of Wisconsin, Madison Wisconsin, USA

${ }^{177}$ Fakultät für Physik und Astronomie, Julius-Maximilians-Universität, Würzburg, Germany

${ }^{178}$ Fakultät für Mathematik und Naturwissenschaften, Fachgruppe Physik, Bergische Universität Wuppertal, Wuppertal, Germany

${ }^{179}$ Department of Physics, Yale University, New Haven Connecticut, USA

${ }^{180}$ Yerevan Physics Institute, Yerevan, Armenia

${ }^{181}$ Centre de Calcul de l'Institut National de Physique Nucléaire et de Physique des Particules (IN2P3), Villeurbanne, France

${ }^{182}$ Academia Sinica Grid Computing, Institute of Physics, Academia Sinica, Taipei, Taiwan

${ }^{\mathrm{a}}$ Deceased.

${ }^{\mathrm{b}}$ Also at Department of Physics, King's College London, London, United Kingdom.

${ }^{\mathrm{c}}$ Also at Institute of Physics, Azerbaijan Academy of Sciences, Baku, Azerbaija.

${ }^{\mathrm{d}}$ Also at Novosibirsk State University, Novosibirsk, Russia.

eAlso at TRIUMF, Vancouver British Columbia, Canada.

${ }_{\mathrm{f}}^{\mathrm{f}}$ Also at Department of Physics \& Astronomy, University of Louisville, Louisville, Kentucky, USA.

${ }^{g}$ Also at Physics Department, An-Najah National University, Nablus, Palestine.

${ }^{\mathrm{h}}$ Also at Department of Physics, California State University, Fresno California, USA.

${ }^{\mathrm{i}}$ Also at Department of Physics, University of Fribourg, Fribourg, Switzerland.

${ }^{\mathrm{j}}$ Also at II Physikalisches Institut, Georg-August-Universität, Göttingen, Germany.

${ }^{\mathrm{k}}$ Also at Departament de Fisica de la Universitat Autonoma de Barcelona, Barcelona, Spain.

${ }^{1}$ Also at Departamento de Fisica e Astronomia, Faculdade de Ciencias, Universidade do Porto, Portugal.

${ }^{\mathrm{m}}$ Also at Tomsk State University, Tomsk, Russia.

${ }^{\mathrm{n}}$ Also at The Collaborative Innovation Center of Quantum Matter (CICQM), Beijing, China.

${ }^{\circ}$ Also at Universita di Napoli Parthenope, Napoli, Italy.

${ }^{\mathrm{p}}$ Also at Institute of Particle Physics (IPP), Canada.

${ }^{\mathrm{q}}$ Also at Horia Hulubei National Institute of Physics and Nuclear Engineering, Bucharest, Romania. 
${ }^{r}$ Also at Department of Physics, St. Petersburg State Polytechnical University, St. Petersburg, Russia.

${ }^{\mathrm{s}}$ Also at Borough of Manhattan Community College, City University of New York, New York City, USA.

${ }^{t}$ Also at Department of Financial and Management Engineering, University of the Aegean, Chios, Greece.

uAlso at Centre for High Performance Computing, CSIR Campus, Rosebank, Cape Town, South Africa.

${ }^{v}$ Also at Louisiana Tech University, Ruston Louisiana, USA.

${ }^{w}$ Also at Institucio Catalana de Recerca i Estudis Avancats, ICREA, Barcelona, Spain.

${ }^{\mathrm{x}}$ Also at Graduate School of Science, Osaka University, Osaka, Japan.

${ }^{y}$ Also at Fakultät für Mathematik und Physik, Albert-Ludwigs-Universität, Freiburg, Germany.

${ }^{\mathrm{z}}$ Also at Institute for Mathematics, Astrophysics and Particle Physics, Radboud University Nijmegen/Nikhef, Nijmegen, Netherlands.

${ }^{\text {aa }}$ Also at Department of Physics, The University of Texas at Austin, Austin Texas, USA.

${ }^{\mathrm{bb}}$ Also at Institute of Theoretical Physics, Ilia State University, Tbilisi, Georgia.

${ }^{c c}$ Also at CERN, Geneva, Switzerland.

${ }^{\mathrm{dd}}$ Also at Georgian Technical University (GTU),Tbilisi, Georgia.

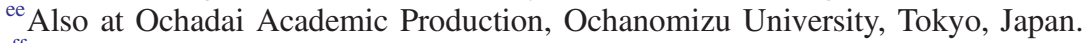

${ }^{\mathrm{ff}}$ Also at Manhattan College, New York, New York, USA.

${ }^{g g}$ Also at Departamento de Física, Pontificia Universidad Católica de Chile, Santiago, Chile.

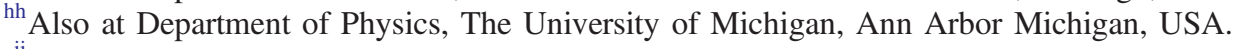

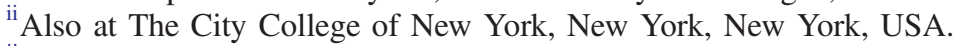

${ }^{\mathrm{jj}}$ Also at School of Physics, Shandong University, Shandong, China.

${ }^{\mathrm{kk}}$ Also at Departamento de Fisica Teorica y del Cosmos and CAFPE, Universidad de Granada, Granada, Portugal.

${ }^{11}$ Also at Department of Physics, California State University, Sacramento California, USA.

${ }^{\mathrm{mm}}$ Also at Moscow Institute of Physics and Technology State University, Dolgoprudny, Russia.

${ }^{\mathrm{nn}}$ Also at Departement de Physique Nucleaire et Corpusculaire, Université de Genève, Geneva, Switzerland.

${ }^{\text {oo }}$ Also at Institut de Física d'Altes Energies (IFAE), The Barcelona Institute of Science and Technology, Barcelona, Spain.

${ }^{\mathrm{pp}}$ Also at School of Physics, Sun Yat-sen University, Guangzhou, China.

${ }^{\mathrm{qq}}$ Also at Institute for Nuclear Research and Nuclear Energy (INRNE) of the Bulgarian Academy of Sciences, Sofia, Bulgaria.

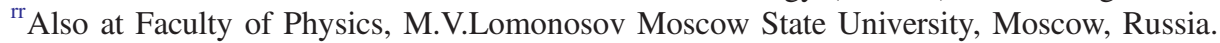

${ }^{\text {ss }}$ Also at National Research Nuclear University MEPhI, Moscow, Russia.

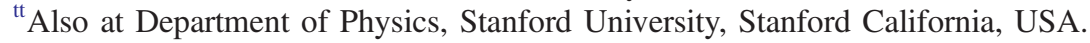

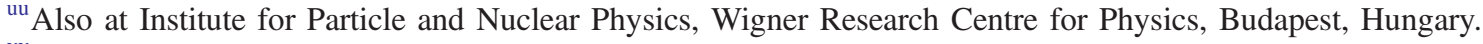

${ }^{\mathrm{vv}}$ Also at Giresun University, Faculty of Engineering, Turkey.

${ }^{\text {ww }}$ Also at CPPM, Aix-Marseille Université and CNRS/IN2P3, Marseille, France.

${ }^{\mathrm{xx}}$ Also at Department of Physics, Nanjing University, Jiangsu, China.

${ }^{y y}$ Also at University of Malaya, Department of Physics, Kuala Lumpur, Malaysia.

${ }^{\mathrm{zz}}$ Also at Institute of Physics, Academia Sinica, Taipei, Taiwan.

${ }^{\text {aaa }}$ Also at LAL, Univ. Paris-Sud, CNRS/IN2P3, Université Paris-Saclay, Orsay, France. 